\title{
GLOBAL SPLITTINGS \\ AND SUPER HARISH-CHANDRA PAIRS FOR AFFINE SUPERGROUPS
}

\author{
FABIO GAVARINI
}

Abstract. This paper dwells upon two aspects of affine supergroup theory, investigating the links among them.

First, the "splitting" properties of affine supergroups are discussed, i.e., special kinds of factorizations they may admit - either globally, or pointwise. Almost everything should be more or less known, but seems to be not as clear in the literature (to the author's knowledge) as it ought to.

Second, a new contribution to the study of affine supergroups by means of super Harish-Chandra pairs is presented (a method already introduced by Koszul, and later extended by other authors). Namely, a new functorial construction $\Psi$ is provided which, with each super Harish-Chandra pair, associates an affine supergroup that is always globally strongly split (in short, gs-split) - thus setting a link with the first part of the paper. One knows that there exists a natural functor $\Phi$ from affine supergroups to super Harish-Chandra pairs. Then we show that the new functor $\Psi$ - which goes the other way round - is indeed a quasi-inverse to $\Phi$, provided we restrict our attention to the subcategory of affine supergroups that are gs-split. Therefore, (the restrictions of) $\Phi$ and $\Psi$ are equivalences between the categories of gs-split affine supergroups and of super Harish-Chandra pairs. Such a result was known in other contexts, such as the smooth differential or the complex analytic one, via different approaches. Nevertheless, the novelty in the present paper lies in the construction of a different functor $\Psi$ and thus extends the result to a much larger setup, with a totally different, more geometrical method. In fact, this method (very concrete, indeed) is universal and characteristic-free and is presented here for the algebro-geometric setting, but actually it can be easily adapted to the frameworks of differential or complex analytic supergeometry.

The case of linear supergroups is treated also as an intermediate, inspiring step.

Some examples, applications and further generalizations are presented at the end of the paper.

\section{Contents}

1. Introduction

2. Preliminaries

3. Splittings

4. Supergroups and super Harish-Chandra pairs

References

Received by the editors September 14, 2013 and, in revised form, April 1, 2014 and April 3, 2014 .

2010 Mathematics Subject Classification. Primary 14M30, 14A22; Secondary 17B20.

The author thanks A. D'Andrea, M. Duflo and R. Fioresi for their priceless suggestions, and most of all, in particular, A. Masuoka for his many valuable comments and remarks. 


\section{INTRODUCTION}

The study of "supergroups" is a chapter of "supergeometry", i.e., geometry in a $\mathbb{Z}_{2}$-graded sense. In particular, the relevant structure sheaves of (commutative) algebras sitting on top of the topological spaces one works with are replaced with sheaves of (commutative) superalgebras.

Every superalgebra $A$ is built from (homogeneous) even and odd elements. It is then natural - especially in the commutative case, when these elements can be thought of as "functions" on some superspace - to look for some "separation of variables" result for $A$, in the form of a "splitting", i.e., a factorization of type $A=\bar{A} \otimes A^{\prime}$ where $\bar{A}$ is a totally even subalgebra and $A^{\prime}$ is a second algebra which encodes the "odd part" of $A$. Actually, in the commutative case the best one can hope for is that $A^{\prime}$ be an algebra freely generated by some subsets of odd elements in $A$, hence $A^{\prime}$ is a Grassmann (super)algebra, i.e., the "polynomial (super)algebra" on some set of odd variables.

When coming to supergeometry, we deal with "superspaces" such as smooth or analytic supermanifolds (in the differential and complex holomorphic setup) or superschemes (in the algebro-geometric framework). Any such superspace can be considered as a classical (i.e. non-super) space - in the appropriate category endowed with a suitable sheaf of commutative superalgebras.

A natural question then arises: can one parallelize this sheaf? In other words, is it globally trivial, in some "natural" sense? For superspaces (in any sense: differential, analytic, etc.) the answer in general is in the negative. Indeed, counterexamples do exist. Instead, if we restrict to supergroups, then the answer in most cases is positive. Indeed, this is the case for real Lie supergroups (see [1, [17, 6]) and for complex analytic supergroups (see [21] and [7]); in the algebro-geometric setting, the best result we are aware of is by Masuoka (see [18]), who proved that for all affine supergroups over fields of characteristic different from 2 the answer still is positive.

It might be worth recalling the analogy with the situation of the tangent bundle on a classical space: for a generic space (manifold, complex analytic variety or scheme) in general it is not parallelizable; for groups instead (real Lie groups, complex analytic Lie groups and group-schemes) it is known to be parallelizable. This might lead us to expect, from scratch, that a similar result can occur with supergroups and their structure sheaf - although this is nothing but a sheer analogy.

Note that in the affine case having a parallelization of the structure sheaf on a superspace $X$ amounts to having a "splitting" of its superalgebra of global sections $\mathcal{O}(X)$. This sets a link with the previously mentioned theme of splitting (commutative) superalgebras, and also leads us to say that $X$ has a "global splitting", or it is globally split, whenever its structure sheaf is parallelizable.

On the other hand, one can study any supergroup $G$, like any superspace, via its functor of points. Then, for each commutative superalgebra $A$ one has the group $G(A)$ of $A$-points of $G$. Such a group may have remarkable "splittings" (in a grouptheoretical sense) on its own; this kind of "pointwise splitting" is often considered in literature (e.g. in Boseck's papers [3], 4], [5]), but must not be confused with the notion of "global splitting".

Roughly speaking, a parallelized "supersheaf" $\mathcal{S}$ over a superspace $X$ is "encoded" by a pair $\left(\mathcal{S}_{\mathbf{0}}, S_{x_{0}}\right)$ where $\mathcal{S}_{\mathbf{0}}$ is the "even part" of $\mathcal{S}$ and $S_{x_{0}}$ is the fiber of $\mathcal{S}$ over some point $x_{0}$; as $\mathcal{S}_{\mathbf{0}}$ is encoded in the classical (i.e. non-super) space $X_{\mathbf{0}}$ 
underlying $X$, one can also use the pair $\left(X_{\mathbf{0}}, S_{x_{0}}\right)$ instead. When $X=G$ is a supergroup, we can take $x_{0}$ to be the identity element in the (classical) group $G_{\mathbf{0}}$ and approximate $S_{x_{0}}$ with the cotangent space at $G_{0}$ in that point; we can also replace this cotangent space with its dual, i.e., the tangent Lie superalgebra $\mathfrak{g}:=$ $\operatorname{Lie}(G)$ of $G$.

This leads us to another - tightly related - way of formulating the problem, namely inquiring whether it is possible (via a "parallelization" of the structure sheaf, etc.) to describe a supergroup $G$ in terms of the pair $\left(G_{\mathbf{0}}, \mathfrak{g}\right)$ which is naturally associated with it. Indeed, this is the core of the problem of studying supergroups via "super Harish-Chandra pairs", as we now explain.

The notion of "super Harish-Chandra pair" (a terminology first found in [9]), or just sHCp in the sequel, was first introduced in the real differential setup, but naturally adapts to the complex analytic or the algebro-geometric context (see, e.g., [21] and [7]). Whatever the setup, an $\mathrm{sHCp}$ is a pair $\left(G_{+}, \mathfrak{g}\right)$ made of a classical group (real Lie, complex analytic, etc.) and a Lie superalgebra obeying natural compatibility constraints. Indeed, the definition itself is tailored in such a way that there exists a natural functor $\Phi$ from the category of supergroups to the category of sHCp's which associates with each supergroup $G$ its sHCp $\left(G_{e v}, \operatorname{Lie}(G)\right)$ made of the "classical subgroup" and the "tangent Lie superalgebra" of $G$. The question is: can one recover a supergroup out of its associated sHCp? In other words, does there exist any functor $\Psi$ from sHCp's to supergroups which could be a quasi-inverse for $\Phi$ ? And if the answer is positive, how explicit is such a functor?

In the real differential framework - i.e. for real Lie supergroups and real smooth sHCp's - Kostant proved (see [15, and also [16]) that $\Phi$ is an equivalence i.e. one has a quasi-inverse for it.

In addition, Vishnyakova (see [21]) fixed both the real smooth and the complex analytic cases.

As to the algebraic setup, more recently Carmeli and Fioresi (see [7]) proved the same result for algebraic affine supergroup schemes (and the corresponding category of sHCp's) over a ground ring $\mathbb{k}$ that is an algebraically closed field of characteristic zero. Indeed, their method - which extends Vishnyakova's idea, so applies to the real smooth and complex analytic setup too - provides an explicit construction of a quasi-inverse functor $\Psi$ for $\Phi$. This was improved by Masuoka (in [19]), who only required that $\mathbb{k}$ be a field whose characteristic is not 2 , and applied his result to a characteristic-free study of affine supergroup schemes. Later on (see [20]), Masuoka and Shibata further extended Koszul's method to work on every commutative ring, via an algebraic version of the notion of sHCp - devised to treat the matter with Hopf (super)algebra techniques.

In the second part of this paper we present a new solution to these problems, providing explicitly a new functor $\Psi$ (different from those by other authors), which does the job; in particular, we also show that any positive answer is possible if and only if we restrict our attention to those (affine) supergroups which are globally strongly split - thus setting a link with the first part of the paper.

The above-mentioned construction of the functor $\Psi$ is made in the setup (and with the language) of algebraic supergeometry. Nevertheless, it is worth stressing that one can easily reformulate everything in the setup (and with the language) of real differential supergeometry or complex analytic supergeometry. In other words, the method presented here also applies, mutatis mutandis, to real or complex Lie supergroups (which, as we mentioned, are known to be all globally split). 
The paper is organized as follows. First (Section 2) we establish the language and notation we need. Then (Section 3) we treat the notions of "splittings" for superalgebras, Hopf superalgebras, superschemes and supergroups; in particular, we present some results about global splittings of supergroups and about their "local" splittings, i.e., splittings on $A$-points. Finally (Section 4), we study the relation between supergroups and super Harish-Candra pairs, and the construction of a functor $\Psi$ which is quasi-inverse to the natural one $\Phi$ associating an sHCp with any supergroup.

\section{Preliminaries}

In this section we introduce some preliminaries of (affine) supergeometry. Classical references for that are [9], 17] and [22, but we shall mainly rely on [6].

Throughout the paper, $\mathbb{k}$ will be a commutative, unital ring.

2.1. Superalgebras, superspaces, supergroups. This subsection is devoted to fix terminology and notation for some basic notions.

2.1.1. Supermodules and superalgebras. A $\mathbb{k}$-supermodule is by definition a $\mathbb{k}-$ module $V$ endowed with a $\mathbb{Z}_{2}$-grading, say $V=V_{\mathbf{0}} \oplus V_{\mathbf{1}}$, where $\mathbb{Z}_{2}=\{\mathbf{0}, \mathbf{1}\}$ is the group with two elements. The $\mathbb{k}$-submodule $V_{\mathbf{0}}$ and its elements are called even, while $V_{\mathbf{1}}$ and its elements odd. By $|x|$ or $p(x)\left(\in \mathbb{Z}_{2}\right)$ we denote the parity of any homogeneous element, defined by the condition $x \in V_{|x|}$.

We call a $\mathbb{k}$-superalgebra any associative, unital $\mathbb{k}$-algebra $A$ which is $\mathbb{Z}_{2}$-graded (as a $\mathbb{k}$-algebra): so $A$ has a $\mathbb{Z}_{2}$-splitting $A=A_{\mathbf{0}} \oplus A_{\mathbf{1}}$, and $A_{\mathbf{a}} A_{\mathbf{b}} \subseteq A_{\mathbf{a}+\mathbf{b}}$. All $\mathbb{k}$-superalgebras form a category, whose morphisms are all those in the category of $\mathbb{k}$-algebras that preserve the unit and the $\mathbb{Z}_{2}$-grading. A Hopf superalgebra over $\mathbb{k}$ is a Hopf algebra $H=H_{\mathbf{0}} \oplus H_{\mathbf{1}}$ in the category of $\mathbb{k}$-superalgebras, where the multiplication in a tensor product $H^{\prime} \underset{\mathbb{k}}{\otimes} H^{\prime \prime}$ is given by

$$
\left(h_{1}^{\prime} \otimes h_{1}^{\prime \prime}\right) \cdot\left(h_{2}^{\prime} \otimes h_{2}^{\prime \prime}\right):=(-1)^{\left|h_{1}^{\prime \prime}\right|\left|h_{2}^{\prime}\right|}\left(h_{1}^{\prime} \cdot h_{2}^{\prime}\right) \otimes\left(h_{1}^{\prime \prime} \cdot h_{2}^{\prime \prime}\right) .
$$

Morphisms among Hopf superalgebras are then the obvious ones.

In the following, if $H$ is any Hopf superalgebra with counit $\epsilon$ we shall write $H^{+}:=\operatorname{Ker}(\epsilon)$.

A superalgebra $A$ is said to be commutative iff $x y=(-1)^{|x||y|} y x$ for all homogeneous $x, y \in A$ and $z^{2}=0$ for all odd $z \in A_{\mathbf{1}}$. We denote by (salg) the category of commutative $\mathbb{k}$-superalgebras; if necessary, we shall stress the role of $\mathbb{k}$ by writing $(\mathrm{salg})_{\mathbb{k}}$. A Hopf superalgebra is said to be commutative if it is such as a superalgebra, and we denote by $(\mathrm{H} \text {-salg })_{\mathbb{k}}$, or simply $(\mathrm{H}$-salg), the category of commutative Hopf $\mathbb{k}$-superalgebras. We shall also denote by $(\mathrm{alg})_{\mathbb{k}}$ - or simply (alg) - the category of (associative) commutative unital $\mathbb{k}$-algebras.

For $A \in(\mathrm{salg})_{\mathbb{k}}, n \in \mathbb{N}$, we call $A_{1}^{[n]}$ the $A_{\mathbf{0}}$-submodule of $A$ spanned by all products $\vartheta_{1} \cdots \vartheta_{n}$ with $\vartheta_{i} \in A_{\mathbf{1}}$ for all $i$, and then $A_{1}^{(n)}$ and $A_{1}^{n}$ respectively the unital $\mathbb{k}^{-}$-subalgebra and the ideal of $A$ generated by $A_{1}^{[n]}$. Similarly we consider $H_{1}^{[n]}, H_{1}^{(n)}, H_{1}^{n}$ for $H \in(\mathrm{H}-\mathrm{salg})_{\mathbb{k}}$.

We also need to consider the following constructions. Given $A=A_{\mathbf{0}} \oplus A_{\mathbf{1}} \in$ $(\mathrm{salg})_{\mathbb{K}}$, let $J_{A}:=\left(A_{\mathbf{1}}\right)$ be the ideal of $A$ generated by $A_{\mathbf{1}}$. Then $J_{A}=A_{\mathbf{1}}^{[2]} \oplus A_{\mathbf{1}}$, and $\bar{A}:=A / J_{A}$ is a commutative superalgebra which is totally even, i.e., $\bar{A} \in$ $(\mathrm{alg})_{\mathbb{k}} ;$ moreover, there is an obvious isomorphism $\bar{A}:=A /\left(A_{\mathbf{1}}\right) \cong A_{\mathbf{0}} / A_{\mathbf{1}}^{[2]}$. Also, 
the construction of $\bar{A}$ is functorial in $A$; and similarly for the constructions of $A_{\mathbf{0}}$ and of $A_{1}^{(n)}$. This yields functors

$$
\overline{(~)},()_{\mathbf{0}}:(\operatorname{salg})_{\mathbb{k}} \longrightarrow(\operatorname{alg})_{\mathbb{k}} \quad \text { and } \quad()_{1}^{(n)}:(\operatorname{salg})_{\mathbb{k}} \longrightarrow(\operatorname{salg})_{\mathbb{k}}
$$

respectively defined on objects by $A \mapsto \bar{A}, A \mapsto A_{\mathbf{0}}, A \mapsto A_{1}^{(n)}(n \in \mathbb{N})$.

On the other hand, there is an obvious functor $\mathcal{J}_{(\mathrm{alg})_{\mathrm{k}}}^{(\mathrm{salg})_{\mathfrak{k}}}:(\mathrm{alg})_{\mathbb{k}} \longrightarrow(\mathrm{salg})_{\mathbb{k}}$ given by taking any commutative $\mathbb{k}$-algebra as a totally even superalgebra; both $\overline{(~)}$ and ()$_{0}$ are retractions of $\mathcal{J}_{(\mathrm{alg})_{\mathrm{k}}}^{(\mathrm{salg})_{\mathrm{k}}}$.

We shall now introduce the affine superschemes, which by definition are representable functors from (salg) to the category (sets) of all sets.

Definition 2.1.2. For any $R \in(\mathrm{salg})_{\mathbb{k}}$, we call spectrum of $R$, denoted $\operatorname{Spec}(R)$ or also $h_{R}$, the representable functor $\underline{\operatorname{Spec}}(R)=h_{R}:(\mathrm{salg})_{\mathbb{k}} \longrightarrow$ (sets) associated with $R$. Explicitly, $h_{R}$ is given on objects by $h_{R}(A):=\operatorname{Hom}_{(\mathrm{salg})_{\mathrm{k}}}(R, A)$ and on arrows by $h_{R}(f)(\phi):=f \circ \phi$. All such spectra are also called affine $\mathbb{k}-$ superschemes. Any affine superscheme is said to be algebraic if its representing (commutative) superalgebra is finitely generated.

When $h_{R}$ is actually a functor from (salg) to (groups), the category of groups, we say that $h_{R}$ is an (affine) group $\mathbb{k}$-superscheme, in short an (affine) $\mathbb{k}$-supergroup; indeed, this is equivalent to the fact that $R$ is a (commutative) Hopf superalgebra, i.e., $R \in(\mathrm{H} \text {-salg })_{\mathbb{k}}$. In other words, the (affine) group superschemes are nothing but the functors from $(\mathrm{salg})_{\mathbb{k}}$ to (groups) which are representable. Any affine $\mathbb{k}^{-}$ supergroup is algebraic if it is such as an affine $\mathbb{k}$-superscheme, i.e., its representing (Hopf) $\mathbb{k}$-superalgebra is of finite type.

All affine $\mathbb{k}$-superschemes form a category, with suitably defined morphisms, denoted by $(\text { assch })_{\mathbb{k}}$ which is isomorphic to the category $(\mathrm{salg})_{\mathbb{k}}^{\circ}$ opposite to $(\mathrm{salg})_{\mathbb{k}}$. An isomorphism $(\text { salg })_{\mathbb{k}}^{\circ} \cong(\text { assch })_{\mathbb{k}} \quad$ is given on objects by $R \mapsto h_{R}$, and we denote its inverse $(\text { assch })_{\mathbb{k}} \cong(\text { salg })_{\mathbb{k}}^{\circ}$ by $X \mapsto \mathcal{O}(X)$. Similarly, all affine $\mathbb{k}^{-}$ supergroups form a category, denoted by (sgroups) $)_{\mathbb{k}}$, isomorphic to the category $(\mathrm{H} \text {-salg })_{\mathbb{k}_{\mathrm{k}}}^{\circ}$ opposite to $(\mathrm{H} \text {-salg })_{\mathbb{k}}$. Explicit isomorphisms are given (with same notation) by restrictions of the previous ones between $(\mathrm{salg})_{\mathbb{k}}^{\circ}$ and $(\operatorname{assch})_{\mathbb{k}}$ respectively.

More generally, we call respectively superset $\mathbb{k}$-functor and supergroup $\mathbb{k}$-functor (possibly dropping the " $\mathbb{k}^{-}$") any functor $X:(\mathrm{salg})_{\mathbb{k}} \longrightarrow$ (sets) and any functor $G:(\text { salg })_{\mathbb{k}} \longrightarrow$ (groups) .

Example 2.1.3. The affine superspace $\mathbb{A}_{\mathbb{k}}^{p \mid q}$, denoted $\mathbb{k}^{p \mid q}$ too, is defined (for $p$, $q \in \mathbb{N})$ as $\mathbb{A}_{\mathbb{k}}^{p \mid q}:=\underline{\operatorname{Spec}}\left(\mathbb{k}\left[x_{1}, \ldots, x_{p}\right] \otimes \mathbb{k}\left[\xi_{1} \ldots \xi_{q}\right]\right)$ where $\mathbb{k}\left[\xi_{1} \ldots \xi_{q}\right]$ is the exterior (or "Grassmann") algebra generated by odd variables $\xi_{1}, \ldots, \xi_{q}$, and $\mathbb{k}\left[x_{1}, \ldots, x_{p}\right]$ the polynomial algebra in $p$ commuting variables.

The superdimension of $\mathbb{A}_{\mathbb{k}}^{p \mid q}$ is easily seen to be $p \mid q$.

Remark 2.1.4. More generally, one can consider the broader notions of (not necessarily affine) superscheme and supergroup, still defined over $(\mathrm{salg})_{\mathbb{k}}$ - see [6] for more details. In the present work, however, we do not need to consider such more general notions.

The next examples turn out to be very important in the sequel. 


\section{Examples 2.1.5.}

(a) Let $V$ be a free $\mathbb{k}$-supermodule, that is, a $\mathbb{k}$-supermodule for which both $V_{\mathbf{0}}$ and $V_{\mathbf{1}}$ are free as $\mathbb{k}$-modules. For any superalgebra $A$ we define $V(A):=$ $(A \otimes V)_{\mathbf{0}}=A_{\mathbf{0}} \otimes V_{\mathbf{0}} \oplus A_{\mathbf{1}} \otimes V_{\mathbf{1}}$. This is a representable functor in the category of superalgebras, whose representing object is the $\mathbb{k}$-superalgebra of polynomial functions on $V$. Hence $V$ can be seen as an affine $\mathbb{k}$-superscheme.

(b) $\mathrm{GL}(V)$ as an affine algebraic supergroup. Let $V$ be a $\mathbb{k}$-supermodule which is free and whose rank, i.e., the pair $\operatorname{rk}(V):=\left(\operatorname{rk}\left(V_{\mathbf{0}}\right), \operatorname{rk}\left(V_{\mathbf{1}}\right)\right)$, is finite, i.e., $r k\left(V_{\mathbf{0}}\right), r k\left(V_{\mathbf{1}}\right) \in \mathbb{N}$. For any $\mathbb{k}$-superalgebra $A$, let $\operatorname{GL}(V)(A):=\operatorname{GL}(V(A))$ be the set of isomorphisms $V(A) \longrightarrow V(A)$. If we fix a homogeneous basis for $V$ and we set $p:=r k\left(V_{\mathbf{0}}\right), q:=r k\left(V_{\mathbf{1}}\right) \in \mathbb{N}$, we have $V \cong \mathbb{K}^{p \mid q}$. Then we also denote $\mathrm{GL}(V)$ with $\mathrm{GL}_{p \mid q}$. Now, $\mathrm{GL}_{p \mid q}(A)$ is the group of invertible $(p, q)$-block matrices - whose size is $(p+q)$ - with diagonal block entries in $A_{\mathbf{0}}$ and off-diagonal block entries in $A_{\mathbf{1}}$. It is known that the functor $\mathrm{GL}(V)$ is representable, so $\operatorname{GL}(V)$ is indeed an affine $\mathbb{k}$-supergroup, and it is also algebraic; see (e.g.), 22, Ch. 3, for further details.

Definition 2.1.6. For any superset $\mathbb{k}$-functor $X:(\mathrm{salg})_{\mathbb{k}} \longrightarrow($ sets $)$, we respectively set

$$
\bar{X}:=X \circ \mathcal{J}_{(\mathrm{alg})_{\mathrm{k}}}^{(\mathrm{salg})_{\mathbb{k}}} \circ \overline{()}, \quad X_{\mathbf{0}}:=X \circ \mathcal{J}_{(\mathrm{alg})_{\mathrm{k}}}^{(\mathrm{salg})_{\mathrm{k}}} \circ()_{0}, \quad X_{1}^{(n)}:=X \circ()_{1}^{(n)}
$$

to denote its composition with the functor

$$
\overline{(~)}:(\operatorname{salg})_{\mathbb{k}} \longrightarrow(\operatorname{alg})_{\mathbb{k}}, \quad()_{\mathbf{0}}:(\operatorname{salg})_{\mathbb{k}} \longrightarrow(\operatorname{alg})_{\mathbb{k}} \quad \text { and } \quad()_{1}^{(n)}:(\operatorname{salg})_{\mathbb{k}} \longrightarrow(\operatorname{alg})_{\mathbb{k}}
$$

$(n \in \mathbb{N})$, followed, in the first two cases, by $\mathcal{J}_{(\mathrm{alg})_{\mathrm{k}}}^{(\mathrm{salg})_{\mathfrak{k}}}:(\operatorname{alg})_{\mathbb{k}} \longrightarrow(\mathrm{salg})_{\mathbb{k}}-$ see Section 2.1.1. Similar notation applies when $X=G$ is in fact a supergroup $\mathbb{k}^{-}$ functor.

2.2. Lie superalgebras. The notion of a Lie superalgebra over a field is well known. In particular, it is entirely satisfactory when the characteristic of the ground field $\mathbb{k}$ is neither 2 nor 3 . However, it is not as satisfactory - in the standard formulation - when that characteristic is either 2 or 3 . This motivates one to introduce the following modified formulation, whose main feature is to describe a "correct" notion of Lie superalgebras as given by the standard notion enriched with an additional piece of structure, namely sort of a "2-mapping" that is a close analogue to the $p$-mapping in a $p$-restricted Lie algebra over a field of characteristic $p>0$.

Definition 2.2.1. Let $\mathfrak{g}=\mathfrak{g}_{0} \oplus \mathfrak{g}_{1}$ be a $\mathbb{k}$-supermodule. We say that $\mathfrak{g}$ is a Lie superalgebra if we have a (Lie super)bracket $[\cdot, \cdot]: \mathfrak{g} \times \mathfrak{g} \longrightarrow \mathfrak{g},(x, y) \mapsto[x, y]$, and a 2-operation $(\cdot)^{\langle 2\rangle}: \mathfrak{g}_{\mathbf{1}} \longrightarrow \mathfrak{g}_{\mathbf{0}}, z \mapsto z^{\langle 2\rangle}$, which satisfy the following properties (for all $x, y \in \mathfrak{g}_{\mathbf{0}} \cup \mathfrak{g}_{\mathbf{1}}, w \in \mathfrak{g}_{\mathbf{0}}, z, z_{1}, z_{2} \in \mathfrak{g}_{\mathbf{1}}$ ):
$[\cdot, \cdot]$ is $\mathbb{k}$-bilinear,
$[w, w]=0$,
$[z,[z, z]]=0$

$$
[x, y]+(-1)^{|x||y|}[y, x]=0 \quad \text { (anti-symmetry); }
$$

$$
(-1)^{|x||z|}[x,[y, z]]+(-1)^{|y||x|}[y,[z, x]]+(-1)^{|z||y|}[z,[x, y]]=0
$$

(Jacobi identity); 
(d) $\quad(\cdot)^{\langle 2\rangle}$ is $\mathbb{k}$-quadratic, i.e., $(c z)^{\langle 2\rangle}=c^{2} z^{\langle 2\rangle}$ for all $c \in \mathbb{k}$;

(e) $\quad\left(z_{1}+z_{2}\right)^{\langle 2\rangle}=z_{1}^{\langle 2\rangle}+\left[z_{1}, z_{2}\right]+z_{2}^{\langle 2\rangle}$;

(f) $\quad\left[z^{\langle 2\rangle}, x\right]=[z,[z, x]]$.

All Lie $\mathbb{k}$-superalgebras form a category, denoted $(\mathrm{sLie})_{\mathbb{k}}$, whose morphisms are the $\mathbb{k}$-linear, graded maps preserving the bracket and the 2 -operation.

Remark 2.2.2. The conditions in Definition 2.2.1 are somewhat redundant, and in some cases may be simplified. For instance, condition (e) yields $\left[z_{1}, z_{2}\right]=$ $\left(z_{1}+z_{2}\right)^{\langle 2\rangle}-z_{1}^{\langle 2\rangle}-z_{2}^{\langle 2\rangle}$ so one could use this as a definition of the Lie bracket on $\mathfrak{g}_{1} \times \mathfrak{g}_{1}$ in terms of the 2-operation. Conversely, when 2 is invertible in $\mathbb{k}$ the 2 operation is recovered from the Lie bracket, via condition (e), as $z^{\langle 2\rangle}=2^{-1}[z, z]$.

Example 2.2.3. Let $V=V_{\mathbf{0}} \oplus V_{\mathbf{1}}$ be a free $\mathbb{k}$-supermodule, and consider $\operatorname{End}(V)$, the endomorphisms of $V$ as an ordinary $\mathbb{k}$-module. This is again a free $\mathbb{k}$-supermodule, $\operatorname{End}(V)=\operatorname{End}(V)_{\mathbf{0}} \oplus \operatorname{End}(V)_{\mathbf{1}}$, where $\operatorname{End}(V)_{\mathbf{0}}$ are the morphisms which preserve the parity, while $\operatorname{End}(V)_{\mathbf{1}}$ are the morphisms which reverse the parity. If $V$ has finite rank, and we choose a basis for $V$ of homogeneous elements (writing first the even ones), then $\operatorname{End}(V)_{\mathbf{0}}$ is the set of all diagonal block matrices, while $\operatorname{End}(V)_{\mathbf{1}}$ is the set of all off-diagonal block matrices. Thus $\operatorname{End}(V)$ is a Lie $\mathbb{k}^{-}$ superalgebra with bracket $[A, B]:=A B-(-1)^{|A||B|} B A$ for all homogeneous $A, B \in \operatorname{End}(V)$ and 2-operation $C^{\langle 2\rangle}:=C C$ for all odd $C$.

The standard example is $V:=\mathbb{k}^{p \mid q}=\mathbb{k}^{p} \oplus \mathbb{k}^{q}$, with $V_{\mathbf{0}}:=\mathbb{k}^{p}$ and $V_{\mathbf{1}}:=\mathbb{k}^{q}$. In this case we also write $\operatorname{End}\left(\mathbb{k}^{m \mid n}\right):=\operatorname{End}(V)$ or $\mathfrak{g l}_{p \mid q}:=\operatorname{End}(V)$.

2.2.4. Functorial presentation of Lie superalgebras. Let $(\mathrm{salg})_{\mathbb{k}}$ be the category of commutative $\mathbb{k}$-superalgebras (see Section 2.1) and (Lie) $)_{\mathbb{k}}$ the category of Lie $\mathbb{k}$ algebras. Any Lie $\mathbb{k}$-superalgebra $\mathfrak{g} \in(\mathrm{sLie})_{\mathbb{k}}$ yields a functor

$$
\mathcal{L}_{\mathfrak{g}}:(\mathrm{salg})_{\mathbb{k}} \longrightarrow(\mathrm{Lie})_{\mathbb{k}}
$$

which is given on objects by

$$
\mathcal{L}_{\mathfrak{g}}(A):=(A \otimes \mathfrak{g})_{\mathbf{0}}=A_{\mathbf{0}} \otimes \mathfrak{g}_{\mathbf{0}} \oplus A_{\mathbf{1}} \otimes \mathfrak{g}_{\mathbf{1}} \quad \text { for all } A \in(\mathrm{salg})_{\mathbb{k}} .
$$

Indeed, $A \otimes \mathfrak{g}$ is a Lie superalgebra (in a suitable sense, on the $\mathbb{k}$-superalgebra $A$ ) on its own, its Lie bracket being defined canonically via sign rules by

$$
\left[a \otimes X, a^{\prime} \otimes X^{\prime}\right]:=(-1)^{|X|\left|a^{\prime}\right|} a a^{\prime} \otimes\left[X, X^{\prime}\right]
$$

and $\mathcal{L}_{\mathfrak{g}}(A)$ is its even part, hence it is a Lie algebra (everything is trivial to verify; see [2], or [6], Proposition 11.2.5, for details). In particular, this applies to the Lie superalgebra $\mathfrak{g}:=\operatorname{End}(V)$, where $V$ is any free $\mathbb{k}$-supermodule. Note also that $\mathrm{GL}(V)$ - see Example 2.1.5 (b) - is then a subfunctor of $\mathcal{L}_{\operatorname{End}(V)}$.

This "functorial presentation" of Lie superalgebras can be adapted to representations too. Indeed, let $V$ be a $\mathfrak{g}$-module, for a Lie superalgebra $\mathfrak{g}$. By definition, $V$ is a $\mathbb{k}$-supermodule, and we have a Lie superalgebra morphism $\phi: \mathfrak{g} \longrightarrow$ $\operatorname{End}(V)$ (the representation map). Now, scalar extension induces a morphism $\operatorname{id}_{A} \otimes \phi: A \otimes \mathfrak{g} \longrightarrow A \otimes \operatorname{End}(V)$ for each $A \in(\mathrm{salg})_{\mathbb{K}}$, whose restriction to the even part gives a morphism $(A \otimes \mathfrak{g})_{0} \longrightarrow(A \otimes \operatorname{End}(V))_{0}$, that is, a morphism $\mathcal{L}_{\mathfrak{g}}(A) \longrightarrow \mathcal{L}_{\operatorname{End}(V)}(A)$ in $(\mathrm{Lie})_{\mathbb{k}}$. The whole construction is natural in $A$, hence it induces a natural transformation of functors $\mathcal{L}_{\mathfrak{g}} \longrightarrow \mathcal{L}_{\text {End }(V)}$. 
In the sequel, we shall call quasi-representable any functor $\mathcal{L}:(\mathrm{salg})_{\mathbb{k}} \longrightarrow(\mathrm{Lie})_{\mathbb{k}}$ for which there exists a Lie $\mathbb{k}$-superalgebra $\mathfrak{g}$ such that $\mathcal{L}=\mathcal{L}_{\mathfrak{g}}$. Any such functor is even representable (in the usual sense) as soon as the $\mathbb{k}$-module $\mathfrak{g}$ is finitely generated projective. Indeed, in this case $\mathfrak{g}$ is a $\mathbb{k}$-direct summand of a finite rank free $\mathbb{k}$-supermodule, say $\mathfrak{f}=\mathfrak{g} \oplus \mathfrak{h}$, thus $\mathfrak{f}^{*} \cong \mathfrak{g}^{*} \oplus \mathfrak{h}^{*}$ and $\mathcal{L}_{\mathfrak{g}}$ is then represented by the commutative $\mathbb{k}^{-}$-superalgebra generated by $\mathfrak{g}^{*}$ inside $S\left(\mathfrak{f}^{*}\right)$.

Finally, note that all this has a natural, non-super counterpart which is obtained by letting "Lie algebras" replace "Lie superalgebras" everywhere.

\subsection{The tangent Lie superalgebra of a supergroup.}

We now quickly recall how to associate a Lie superalgebra with a supergroup scheme. Further details can be found in [6], $\S \S 11.2-5$.

Let $A \in(\mathrm{salg})$ and let $A[\varepsilon]:=A[x] /\left(x^{2}\right)$ be the superalgebra of dual numbers over $A$, in which $\varepsilon:=x \bmod \left(x^{2}\right)$ is taken to be even. Then $A[\varepsilon]=A \oplus A \varepsilon$, and there are two natural morphisms $i_{A}: A \longrightarrow A[\varepsilon], a \stackrel{i_{A}}{\mapsto} a$, and $p_{A}: A[\varepsilon] \longrightarrow A$, $\left(a+a^{\prime} \varepsilon\right) \stackrel{p_{A}}{\mapsto} a$, such that $p_{A} \circ i_{A}=i d_{A}$.

Definition 2.3.1. Given a supergroup $\mathbb{k}$-functor $G:$ (salg) $)_{\mathbb{k}} \longrightarrow$ (groups), let $G\left(p_{A}\right): G(A(\varepsilon)) \longrightarrow G(A)$ be the morphism associated with $p_{A}: A[\varepsilon] \longrightarrow A$. There then exists a unique functor $\operatorname{Lie}(G):(\operatorname{salg})_{\mathbb{k}} \longrightarrow($ sets $)$ given on objects by $\operatorname{Lie}(G)(A):=\operatorname{Ker}\left(G(p)_{A}\right)$.

The key fact is that when $G$ is a supergroup $\operatorname{Lie}(G)$ is a Lie algebra valued functor, i.e., a functor $\operatorname{Lie}(G):(\mathrm{salg})_{\mathbb{k}} \longrightarrow(\mathrm{Lie})_{\mathbb{k}}$. This is by no means evident, since the very definition only assures that that functor is group-valued. In fact, stating that $\operatorname{Lie}(G)$ is actually Lie algebra valued requires a non-trivial proof (like in the classical case). For this we refer to [6], Ch. 11 (with the few adaptations needed for the present setup), and restrict ourselves to quickly sketching here the main steps.

The Lie structure on any object $\operatorname{Lie}(G)(A)$ is introduced as follows. First, define the adjoint action of $G$ on $\operatorname{Lie}(G)$ as given, for every $A \in(\operatorname{salg})_{\mathbb{k}}$, by

$$
A d: G(A) \longrightarrow \operatorname{GL}(\operatorname{Lie}(G)(A)), \quad \operatorname{Ad}(g)(x):=G(i)(g) \cdot x \cdot(G(i)(g))^{-1}
$$

for all $g \in G(A), x \in \operatorname{Lie}(G)(A)$. Second, define the adjoint morphism ad as

$$
\text { ad }:=\operatorname{Lie}(A d): \operatorname{Lie}(G) \longrightarrow \operatorname{Lie}(\operatorname{GL}(\operatorname{Lie}(G))):=\operatorname{End}(\operatorname{Lie}(G))
$$

and finally define $[x, y]:=\operatorname{ad}(x)(y)$ for all $x, y \in \operatorname{Lie}(G)(A)$. Then we have the following:

Proposition 2.3.2. Given $G \in(\text { sgroups })_{\mathbb{k}}$, let $\omega_{e}(G):=\mathcal{O}(G)^{+} /\left(\mathcal{O}(G)^{+}\right)^{2}$ and $\mathfrak{g}:=T_{e}(G)=\omega_{e}(G)^{*}=H_{\mathbb{k}}\left(\omega_{e}(G), \mathbb{k}\right)$ be the cotangent and tangent supermodule to $G$ at the unit $e \in G$.

(a) $\operatorname{Lie}(G)$ with the bracket $[\cdot, \cdot]$ above yields a Lie algebra valued functor

$$
\operatorname{Lie}(G):(\operatorname{salg})_{\mathbb{k}} \longrightarrow(\mathrm{Lie})_{\mathbb{k}} .
$$

(b) If Lie $(G)$ is quasi-representable, namely it is of the form $\operatorname{Lie}(G)=\mathcal{L}_{\mathfrak{p}}$ (see Section 2.2.4), then $\mathfrak{p}$ identifies with $\mathfrak{g}$ and the latter is endowed with a canonical structure of Lie $\mathbb{k}$-superalgebra.

(c) Lie $(G)$ is quasi-representable if and only if $\omega_{e}(G)$ is finitely generated projective (over $\mathbb{k})$. When this is the case, Lie $(G)$ is actually representable. 
Proof. Claim (a), i.e., the fact that $\operatorname{Lie}(G)$ with the bracket $[\cdot, \cdot]$ considered above is a Lie algebra valued functor, is a well-known fact; cf. [6], $\S 11.4$ (for instance) for further details.

As to claim (b), it is also standard (cf. [6], §11.2) that if $\operatorname{Lie}(G)=\mathcal{L}_{\mathfrak{p}}$, then $\mathfrak{p}$ necessarily identifies with $\mathfrak{g}:=T_{e}(G)$, and then the existence of a "Lie structure" on $\operatorname{Lie}(G)=\mathcal{L}_{\mathfrak{g}}$ endows $\mathfrak{p}=\mathfrak{g}$ with a structure of "Lie $\mathbb{k}$-superalgebra" in the usual "weak sense"; i.e., $\mathfrak{g}$ has a Lie superbracket for which conditions $(a)-(b)-(c)$ in Definition 2.2.1 are fulfilled. In addition, one has similar, canonical identifications $\operatorname{Lie}(G)=\mathcal{L}_{\mathfrak{g}^{\prime}}$ and $\operatorname{Lie}(G)=\mathcal{L}_{\mathfrak{g}^{\prime \prime}}$ where $\mathfrak{g}^{\prime}:=\operatorname{Der}_{\mathbb{k}}(\mathcal{O}(G), \mathbb{k})$ is the $\mathbb{k}$-superalgebra of $\mathbb{k}$-valued superderivations of $\mathcal{O}(G)$ and $\mathfrak{g}^{\prime \prime}:=\operatorname{Der}_{\mathbb{k}}^{\ell}(\mathcal{O}(G))$ is the $\mathbb{k}$-superalgebra of left-invariant superderivations of $\mathcal{O}(G)$ into itself. Also, both $\mathfrak{g}^{\prime}$ and $\mathfrak{g}^{\prime \prime}$ bear structures of Lie $\mathbb{k}$-superalgebras which are isomorphic to that of $\mathfrak{g}$ (yielding the Lie algebra structure on each $\operatorname{Lie}(G)(A)$, for $\left.A \in(\mathrm{salg})_{\mathbb{K}}\right)$ - see e.g. [6], $\S \S 11.3-6$; there $G$ is assumed to be algebraic, but the arguments (taken from classical sources, such as 8, Ch. II, §4) only require our assumption in (b).

What we still need to fix is that, under the assumption in $(b), \mathfrak{g}:=T_{e}(G)$ is also endowed with a 2 -operation such that $\mathfrak{g}$ is a Lie $\mathbb{k}$-superalgebra in the sense of Definition 2.2.1. Actually, we introduce such a 2-operation on $\mathfrak{g}^{\prime \prime}$ and then use the previous isomorphism(s) to "transfer" such a structure onto $\mathfrak{g}$ (and onto $\left.\mathfrak{g}^{\prime}\right)$ as well. Indeed, the Lie bracket in $\mathfrak{g}^{\prime \prime}:=\operatorname{Der}_{\mathbb{k}}^{\ell}(\mathcal{O}(G))$ is given by $[X, Y]=$ $X \circ Y-(-1)^{|X||Y|} Y \circ X$; in addition, looking at $Z \in \mathfrak{g}_{1}$ as an (odd) left-invariant superderivation of $\mathcal{O}(G)$ one sees at once that $Z^{2}=Z \circ Z$ is an even left-invariant superderivation, i.e., $Z^{2} \in \mathfrak{g}_{\mathbf{0}}$. Then $\mathfrak{g}_{1} \longrightarrow \mathfrak{g}_{\mathbf{0}}, Z \mapsto Z^{\langle 2\rangle}:=Z^{2}$, is well defined and yields a 2 -operation in $\mathfrak{g}$ that along with $[\cdot, \cdot]$ makes it into a Lie $\mathbb{k}$-superalgebra as desired (i.e., in the sense of Definition 2.2.1).

For claim (c) the "if" part is well known again. If $\omega_{e}(G)$ is finitely generated projective, then the same holds true for $\mathfrak{g}=\omega_{e}(G)^{*}$, hence (see Section 2.2.4) the functor $\operatorname{Lie}(G)=\mathcal{L}_{\mathfrak{g}}$ is representable. As to the "only if" part, here is a proof (kindly suggested to the author by Professor Masuoka).

First, by definition of "quasi-representable" (see Section 2.2.4) and by claim (b) above we have that $\operatorname{Lie}(G)$ is quasi-representable if and only if there exist isomorphisms (natural in $R \in(\mathrm{salg})_{\mathbb{k}}$ )

$$
\operatorname{Lie}(G)(R) \cong \mathcal{L}_{\mathfrak{g}}(R):=\left(\mathfrak{g} \otimes_{\mathbb{k}} R\right)_{\mathbf{0}}=\left(\mathfrak{g}_{\mathbf{0}} \otimes_{\mathbb{k}} R_{\mathbf{0}}\right) \oplus\left(\mathfrak{g}_{\mathbf{1}} \otimes_{\mathbb{k}} R_{\mathbf{1}}\right) .
$$

On the other hand, definitions give $\operatorname{Lie}(G)(R) \cong \operatorname{Hom}_{(\operatorname{smod})_{\mathbf{k}}}\left(\omega_{e}(G), R\right)$ where $(\operatorname{smod})_{\mathbb{k}}$ denotes the category of $\mathbb{k}$-supermodules - so that

$\operatorname{Hom}_{(\operatorname{smod})_{\mathrm{k}}}\left(\omega_{e}(G), R\right) \cong \operatorname{Hom}_{(\bmod )_{\mathrm{k}}}\left(\left(\omega_{e}(G)\right)_{\mathbf{0}}, R_{\mathbf{0}}\right) \oplus \operatorname{Hom}_{(\bmod )_{\mathbb{k}}}\left(\left(\omega_{e}(G)\right)_{\mathbf{1}}, R_{\mathbf{1}}\right)$ where now $(\bmod )_{\mathbb{k}}$ denotes the category of $\mathbb{k}$-modules. Thus $(2.1)$ above reads (for all $R$, etc.)

$$
\begin{aligned}
\operatorname{Hom}_{(\bmod )_{\mathfrak{k}}}\left(\left(\omega_{e}(G)\right)_{\mathbf{0}}, R_{\mathbf{0}}\right) \oplus \operatorname{Hom}_{(\bmod )_{\mathbb{k}}}\left(\left(\omega_{e}(G)\right)_{\mathbf{1}}, R_{\mathbf{1}}\right) & \\
& \cong\left(\mathfrak{g}_{\mathbf{0}} \otimes_{\mathbb{k}} R_{\mathbf{0}}\right) \oplus\left(\mathfrak{g}_{\mathbf{1}} \otimes_{\mathbb{k}} R_{\mathbf{1}}\right) .
\end{aligned}
$$

Given $M \in(\bmod )_{\mathbb{k}}$ we associate with it a couple of (super)commutative $\mathbb{k}^{-}$ superalgebras $M_{+}$and $M_{-}$defined as follows. As $\mathbb{k}$-algebras they both are the central extension of $\mathbb{k}$ by $M$ (that is, $M_{+}:=\mathbb{k} \oplus M=: M_{-}$with $m^{\prime} m^{\prime \prime}=0$ for $\left.m^{\prime}, m^{\prime \prime} \in M\right)$, but the $\mathbb{Z}_{2}$-grading is different, namely

$$
\left(M_{+}\right)_{0}:=\mathbb{k} \oplus M, \quad\left(M_{+}\right)_{1}:=\{0\}, \quad\left(M_{-}\right)_{0}:=\mathbb{k}, \quad\left(M_{-}\right)_{1}:=M .
$$


Now assume that $\operatorname{Lie}(G)$ is quasi-representable, hence (2.2) holds true. For $R:=M_{+}$this gives

$\operatorname{Hom}_{(\bmod )_{\mathbb{k}}}\left(\left(\omega_{e}(G)\right)_{\mathbf{0}}, R_{\mathbf{0}}\right)=\operatorname{Hom}_{(\bmod )_{\mathbb{k}}}\left(\left(\omega_{e}(G)\right)_{\mathbf{0}}, \mathbb{k}\right) \oplus \operatorname{Hom}_{(\bmod )_{\mathbf{k}}}\left(\left(\omega_{e}(G)\right)_{\mathbf{0}}, M\right)$ and $\mathfrak{g}_{\mathbf{0}} \otimes_{\mathbb{k}} R_{\mathbf{0}}=\mathfrak{g}_{\mathbf{0}} \otimes_{\mathbb{k}}(\mathbb{k} \oplus M)=\left(\mathfrak{g}_{\mathbf{0}} \otimes_{\mathbb{k}} \mathbb{k}\right) \oplus\left(\mathfrak{g}_{\mathbf{0}} \otimes_{\mathbb{k}} M\right)$, whereas

$$
\operatorname{Hom}_{(\bmod )_{\mathrm{k}}}\left(\left(\omega_{e}(G)\right)_{\mathbf{1}}, R_{\mathbf{1}}\right)=\{0\} \quad, \quad \mathfrak{g}_{\mathbf{1}} \otimes_{\mathbb{k}} R_{\mathbf{1}}=\{0\} .
$$

Therefore condition (2.2) reads

$$
\operatorname{Hom}_{(\bmod )_{\mathbf{k}}}\left(\left(\omega_{e}(G)\right)_{\mathbf{0}}, \mathbb{k}\right) \oplus \operatorname{Hom}_{(\bmod )_{\mathfrak{k}}}\left(\left(\omega_{e}(G)\right)_{\mathbf{0}}, M\right) \cong\left(\mathfrak{g}_{\mathbf{0}} \otimes_{\mathbb{k}} \mathbb{k}\right) \oplus\left(\mathfrak{g}_{\mathbf{0}} \otimes_{\mathbb{k}} M\right)
$$

and eventually (as $\operatorname{Hom}_{(\bmod )_{\mathfrak{k}}}\left(\left(\omega_{e}(G)\right)_{\mathbf{0}}, \mathbb{k}\right)=\left(\left(\omega_{e}(G)\right)_{\mathbf{0}}\right)^{*}=\left(\left(\omega_{e}(G)\right)^{*}\right)_{\mathbf{0}}=$ $\left.\mathfrak{g}_{0}=\mathfrak{g}_{0} \otimes_{\mathbb{k}} \mathbb{k}\right)$

$$
\operatorname{Hom}_{(\bmod )_{\mathbf{k}}}\left(\left(\omega_{e}(G)\right)_{\mathbf{0}}, M\right) \cong \mathfrak{g}_{\mathbf{0}} \otimes_{\mathbb{k}} M
$$

This last condition is natural in $M$. This together with the fact that the functor $M \mapsto \mathfrak{g} \otimes_{\mathbb{k}} M$ preserves surjections, implies that $\left(\omega_{e}(G)\right)_{\mathbf{0}}$ is $\mathbb{k}$-projective.

For $R:=M_{-}$we can repeat the same argument. We find

$$
\operatorname{Hom}_{(\bmod )_{\mathrm{k}}}\left(\left(\omega_{e}(G)\right)_{\mathbf{0}}, R_{\mathbf{0}}\right)=\operatorname{Hom}_{(\bmod )_{\mathbf{k}}}\left(\left(\omega_{e}(G)\right)_{\mathbf{0}}, \mathbb{k}\right)
$$

and $\operatorname{Hom}_{(\bmod )_{\mathfrak{k}}}\left(\left(\omega_{e}(G)\right)_{\mathbf{1}}, R_{\mathbf{1}}\right)=\operatorname{Hom}_{(\bmod )_{\mathbb{k}}}\left(\left(\omega_{e}(G)\right)_{\mathbf{1}}, M\right)$, while $\mathfrak{g}_{\mathbf{0}} \otimes_{\mathbb{k}} R_{\mathbf{0}}=$ $\mathfrak{g}_{0} \otimes_{\mathbb{k}} \mathbb{k} \cong \mathfrak{g}_{0}, \mathfrak{g}_{1} \otimes_{\mathbb{k}} R_{1}=\mathfrak{g}_{1} \otimes_{\mathbb{k}} M$. Thus condition (2.2) now reads

$$
\operatorname{Hom}_{(\bmod )_{\mathbf{k}}}\left(\left(\omega_{e}(G)\right)_{\mathbf{0}}, \mathbb{k}\right) \oplus \operatorname{Hom}_{(\bmod )_{\mathfrak{k}}}\left(\left(\omega_{e}(G)\right)_{\mathbf{1}}, M\right) \cong\left(\mathfrak{g}_{\mathbf{0}} \otimes_{\mathbb{k}} \mathbb{k}\right) \oplus\left(\mathfrak{g}_{\mathbf{1}} \otimes_{\mathbb{k}} M\right)
$$

and then eventually (like before)

$$
\operatorname{Hom}_{(\bmod )_{\mathrm{k}}}\left(\left(\omega_{e}(G)\right)_{1}, M\right) \cong \mathfrak{g}_{\mathbf{1}} \otimes_{\mathbb{k}} M .
$$

As $(2.4)$ is natural in $M$, we can now argue like above to infer that $\left(\omega_{e}(G)\right)_{1}$ is $\mathbb{k}$-projective.

The outcome is that $\omega_{e}(G)=\left(\omega_{e}(G)\right)_{0} \oplus\left(\omega_{e}(G)\right)_{1}$ is $\mathbb{k}$-projective.

Now let $\pi: F=\bigoplus_{i \in I} \mathbb{k} \longrightarrow\left(\omega_{e}(G)\right)_{0}$ be a $\mathbb{k}$-linear surjection from some free $\mathbb{k}$-module $F=\bigoplus_{i \in I}$ of rank $|I|$ onto $\left(\omega_{e}(G)\right)_{0}$. By projectivity of $\left(\omega_{e}(G)\right)_{0}$ there exists a splitting $\sigma:\left(\omega_{e}(G)\right)_{\mathbf{0}} \longrightarrow F$ of $\pi$. Then $\sigma \in \operatorname{Hom}_{(\bmod )_{\mathrm{k}}}\left(\left(\omega_{e}(G)\right)_{\mathbf{0}}, F\right) \cong$ $\mathfrak{g}_{\mathbf{0}} \otimes_{\mathbb{k}} F \cong \mathfrak{g}_{\mathbf{0}} \otimes_{\mathbb{k}}\left(\bigoplus_{i \in I} \mathbb{k}\right)$ - by (2.3) with $M:=F$ - hence there exists some finite index subset $J \subseteq I$ such that $\sigma$ actually belongs to $\mathfrak{g}_{\mathbf{0}} \otimes_{\mathbb{k}}\left(\bigoplus_{i \in J} \mathbb{k}\right)$, which means that the image of $\sigma$ is contained in $F^{\prime}:=\bigoplus_{i \in J} \mathbb{k}$. But then the restriction of $\pi$ to $F^{\prime}$ is still surjective, hence $\left(\omega_{e}(G)\right)_{0}$ is finitely generated.

An entirely similar analysis shows that $\left(\omega_{e}(G)\right)_{0}$ is finitely generated as well.

In the following we are interested in affine $\mathbb{k}$-supergroups of a specific class, characterized in terms of $\operatorname{Lie}(G)$, as the following definition (not very restrictive, indeed) specifies:

Definition 2.3.3. We call fine any affine $\mathbb{k}$-supergroup $G \in(\text { sgroups })_{\mathbb{k}}$ whose associated functor $\operatorname{Lie}(G)$ is quasi-representable, say $\operatorname{Lie}(G)=\mathcal{L}_{\mathfrak{g}}$, for some Lie $\mathbb{k}$-superalgebra $\mathfrak{g}$ whose odd part $\mathfrak{g}_{1}$ is free of finite rank as a $\mathbb{k}$-supermodule. We denote by (fsgroups) $)_{\mathbb{k}}$ the full subcategory of (sgroups) $)_{\mathbb{k}}$ whose objects are all fine $\mathbb{k}$-supergroups. 


\section{Splittings}

In this section we consider the notion of "global splitting" - roughly, a "separation of variables" property — for superalgebras, Hopf superalgebras, (affine) superschemes and supergroups. We shall see that if $\mathbb{k}$ is a field, then all (affine) $\mathbb{k}$-supergroups do admit "global splittings". This is essentially due to a result by Masuoka on the splitting of commutative Hopf superalgebras over a field.

We shall also introduce some other (easy, yet interesting) "splitting results" for the $A$-points of a $\mathbb{k}$-supergroup when $A$ ranges in special subcategories of $(\mathrm{salg})_{\mathbb{k}}$.

\subsection{Augmentations and split superalgebras.}

In the following, we shall think of $\mathbb{k}$ as being a totally even superalgebra, i.e., we identify $\mathbb{k}$ with $\mathcal{J}_{(\mathrm{alg})_{\mathrm{k}}}^{(\mathrm{salg})_{\mathrm{k}}}(\mathbb{k})$ - see Section 2.1.1,

3.1.1. Augmentations for superalgebras and related constructions. For any superalgebra $A \in(\mathrm{salg})_{\mathbb{k}}$, we call augmentation of $A$ any morphism of $\mathbb{k}$-superalgebras $\underline{\epsilon}: A \longrightarrow \mathbb{k}$. We denote by (a-salg) $)_{\mathbb{k}}$ the category of "augmented (commutative) superalgebras". Its objects are pairs $(A, \underline{\epsilon})$ where $A \in(\mathrm{salg})_{\mathbb{k}}$ and $\underline{\epsilon}$ is an augmentation of $A$, and its morphisms $\left(A^{\prime}, \underline{\epsilon}^{\prime}\right) \longrightarrow\left(A^{\prime \prime}, \underline{\epsilon}^{\prime \prime}\right)$ are given by morphisms $\phi: A^{\prime} \longrightarrow A^{\prime \prime}$ in $(\operatorname{salg})_{\mathbb{k}}$ such that $\underline{\epsilon}^{\prime \prime} \circ \phi=\underline{\epsilon}$. We also identify $(A, \underline{\epsilon}) \cong A$.

Given $(A, \underline{\epsilon}) \in(\text { a-salg })_{\mathbb{k}}$ one has $\operatorname{Ker}(\underline{\epsilon})=A_{\mathbf{0}}^{+} \oplus A_{\mathbf{1}}$ where $A_{\mathbf{0}}^{+}:=\operatorname{Ker}(\underline{\epsilon}) \cap A_{\mathbf{0}}$. Define $W^{A}:=A_{\mathbf{1}} / A_{\mathbf{0}}^{+} A_{\mathbf{1}}$ and let $\wedge W^{A}$ be the exterior $\mathbb{k}$-algebra of $W^{A}$; recall also that $A_{\mathbf{1}}^{(1)}$ is the unital $\mathbb{k}^{-}$-subalgebra of $A$ generated by $A_{\mathbf{1}}$ (see Section 2.1.1). Then $\bar{A}:=A / J_{A}, A_{1}^{(1)}$ and $\bigwedge W^{A}$ all inherit from $(A, \underline{\epsilon})$ a natural structure of augmented $\mathbb{k}$-superalgebra.

It follows also that both $\bar{A} \otimes_{\mathbb{k}} A_{1}^{(1)}$ and $\bar{A} \otimes_{\mathbb{k}} \wedge W^{A}$ have a natural structure of commutative, unital, augmented $\mathbb{k}$-superalgebra, i.e., $\bar{A} \otimes_{\mathbb{k}} A_{1}^{(1)}, \bar{A} \otimes_{\mathbb{k}} \wedge W^{A} \in$ $(\mathrm{a}-\mathrm{salg})_{\mathbb{k}}$.

Definition 3.1.2. Given any $A \in(\text { a-salg })_{\mathbb{k}}$, we say that it is weakly split if there exists a section $\sigma_{A}: \bar{A} \longleftrightarrow A$ of the projection $\pi_{A}: A \longrightarrow \bar{A}-$ both being meant as morphisms in (a-salg) $)_{\mathbb{k}}$. All pairs $\left(A, \sigma_{A}\right)$ as above form a category, denoted by (wksp-salg $)_{\mathbb{k}}$, or just (wksp-salg), where morphisms are all those in (a-salg) $)_{\mathbb{k}}$ which are compatible (in the obvious sense) with the sections.

Remark 3.1.3. Given $\left(A, \sigma_{A}\right) \in(\text { wksp-salg })_{\mathbb{k}}$, write $\bar{A}=\sigma_{A}(\bar{A}) \subseteq A$. Then the multiplication map $m_{A}$ in $A$ yields an $\bar{A}$-linear projection $\bar{A} \otimes_{\mathbb{k}} A_{1}^{(1)} \stackrel{m_{A}^{\prime}}{\longrightarrow} A$.

The above remark shows that any weakly split superalgebra $A$ can be recovered as a quotient - in the category of $\bar{A}$-modules, via the multiplication map — of $\bar{A} \otimes_{\mathbb{k}} A_{1}^{(1)}$. This invites us to consider those cases when this description is "optimal", which leads naturally to the next definition.

\section{Definition 3.1.4.}

(a) Any $\left(A, \sigma_{A}\right) \in(\text { wksp-salg })_{\mathbb{k}}$ is said to be split if the natural $\bar{A}$-linear morphism (see above) $\bar{A} \otimes_{\mathbb{k}} A_{1}^{(1)} \stackrel{m_{A}^{\prime}}{\longrightarrow} A$ induced by multiplication in $A$ is an isomorphism in (wksp-salg $)_{\mathbb{k}}$. We denote by (spl-salg) $)_{\mathbb{k}}$ the full subcategory of $(\text { wksp-salg })_{\mathbb{k}}$ of all split $\mathbb{k}$-superalgebras. 
(b) Any augmented superalgebra $(A, \underline{\epsilon}) \in(\text { a-salg })_{\mathbb{k}}$ is said to be strongly split if there exists an isomorphism $\zeta: A \stackrel{\cong}{\leftrightarrows} \otimes_{\mathbb{k}} \wedge W^{A}$ in $(\mathrm{a}-\mathrm{salg})_{\mathbb{k}}$. We denote by $(\text { stsp-salg })_{\mathbb{k}}$ the full subcategory of $(\mathrm{a}-\mathrm{salg})_{\mathbb{k}}$ given by all strongly split $\mathbb{k}$-superalgebras.

We now introduce another special subclass of (commutative) superalgebras.

Let $\mathcal{A} \in(\mathrm{alg})_{\mathbb{k}}$ and let $M$ be an $\mathcal{A}$-module. Then $A_{\mathcal{A}, M}:=\mathcal{A} \oplus M$ has a natural structure of unital, commutative $\mathbb{k}$-superalgebras defined as follows: the $\mathbb{Z}_{2}$-splitting is given by $\left(A_{\mathcal{A}, M}\right)_{0}:=\mathcal{A}, \quad\left(A_{\mathcal{A}, M}\right)_{1}:=M$, and the $\mathbb{k}$-algebra structure is the unique one such that $\mathcal{A}$ is a $\mathbb{k}$-subalgebra, $M \cdot M:=\{0\}$ and $\alpha \cdot m:=\alpha . m=: m \cdot \alpha$ for all $\alpha \in \mathcal{A}, m \in M$, where $\alpha . m$ is given by the $\mathcal{A}$-action on $M$. In a formula,

$$
(\alpha+m) \cdot\left(\alpha^{\prime}+m^{\prime}\right):=\alpha \alpha^{\prime}+\left(\alpha \cdot m^{\prime}+\alpha^{\prime} . m\right) \quad \forall \alpha, \alpha^{\prime} \in \mathcal{A}, m, m^{\prime} \in M .
$$

By construction, the $\mathbb{k}$-superalgebra $A:=A_{\mathcal{A}, M}$ has the property that $A_{1}^{2}=$ $\{0\}$. Conversely, let $A \in(\mathrm{salg})_{\mathbb{k}}$ be such that $A_{1}^{2}=\{0\}$. Then $A$ is of the previous form, namely $A:=A_{\mathcal{A}, M}$ for $\mathcal{A}:=A_{0}$ and $M:=A_{1}$.

Definition 3.1.5. We call an augmented central extension ( $\mathbb{k}$-superalgebra), or simply a central extension, any $(A, \epsilon) \in(\mathrm{a}-\mathrm{salg})_{\mathbb{k}}$ which (as a superalgebra) is of the form $A=A_{\mathcal{A}, M}$ as above with $\epsilon(M)=0$ - in other words, such that $A_{1}^{2}=\{0\}$ and $\epsilon\left(A_{1}\right)=\{0\}$. We denote by (cex-salg $)_{\mathbb{k}}$ the full subcategory of $(\mathrm{salg})_{\mathbb{k}}$ whose objects are all the central extension $\mathbb{k}$-superalgebras.

The next (easy) result shows the links between these special subcategories of $(\operatorname{salg})_{\mathbb{k}}$.

\section{Proposition 3.1.6.}

(a) The category (stsp-salg) $)_{\mathbb{k}}$ identifies in a natural way with a subcategory of $(\mathrm{spl}-\mathrm{salg})_{\mathbb{k}}$, and similarly (spl-salg) $)_{\mathbb{k}}$ identifies with a subcategory of (wksp-salg) ${ }_{\mathbb{k}}$. In other words, all strongly split $\mathbb{k}$-superalgebras are split, and all split $\mathbb{k}$-superalgebras are weakly split.

(b) The category (cex-salg) $)_{\mathbb{k}}$ identifies in a natural way with a subcategory of $(\mathrm{spl}-\mathrm{salg})_{\mathbb{k}}$, i.e., all central extension $\mathbb{k}$-superalgebras are (naturally) split.

Proof. (a) Given $A \in$ (stsp-salg) $)_{\mathbb{k}}$, by the isomorphism $\zeta: A \cong \bar{A} \otimes_{\mathbb{k}} \wedge W^{A}$ one has $A_{\mathbf{1}}=\bar{A} \otimes_{\mathbb{k}}\left(\underset{\text { odd } s}{\oplus}\left(W^{A}\right)^{\wedge s}\right)$ hence $A_{1}^{(1)}=\mathbb{k} \oplus\left(\bar{A} \otimes_{\mathbb{k}}\left(\wedge W^{A}\right)^{+}\right)$, and then an easy calculation shows that $A \in(\text { spl-salg })_{\mathbb{k}}$, as expected. Moreover, if $A \in(\mathrm{spl}-\mathrm{salg})_{\mathbb{k}}$, then the monomorphism $\bar{A} \longleftrightarrow \bar{A} \otimes_{\mathbb{k}} A_{1}^{(1)}(\bar{a} \mapsto \bar{a} \otimes 1)$ composed with the isomorphism $\bar{A} \otimes_{\mathbb{k}} A_{1}^{(1)} \longleftrightarrow A$ (inverse to the one given by definition) yields a section $\bar{A} \longleftrightarrow A$ of $\pi_{A}: A \longrightarrow \bar{A}$, so that $A \in(\text { wksp-salg) })_{\mathbb{k}}$.

(b) Let $A=A_{\mathbf{0}} \oplus A_{\mathbf{1}} \in$ (cex-salg $)_{\mathbb{k}}$, so that $A_{\mathbf{1}}^{2}=\{0\}$. Then definitions give $J_{A}:=\left(A_{1}\right)=A_{1}^{2} \oplus A_{1}=A_{1}$ hence $\bar{A}:=A / J_{A}=A / A_{1}=A_{\mathbf{0}}$, and $A_{1}^{(1)}=\mathbb{k} \oplus A_{1}$. Thus $A=A_{\mathbf{0}} \oplus A_{\mathbf{1}}=\bar{A} \oplus A_{\mathbf{1}} \cong \bar{A} \otimes_{\mathbb{k}} A_{\mathbf{1}}^{(1)}$, so that $A \in(\text { spl-salg })_{\mathbb{k}}$.

3.1.7. Strongly split Hopf superalgebras. Any commutative Hopf $\mathbb{k}$-superalgebra, say $H \in(\mathrm{H}-\mathrm{salg})_{\mathbb{k}}$, is naturally augmented, in the sense of Section 3.1.1, its augmentation being the counit. So $(\mathrm{H}-\mathrm{salg})_{\mathbb{k}}$ naturally identifies with a subcategory of $(\mathrm{a}-\mathrm{salg})_{\mathbb{k}}$, and all constructions therein make sense for Hopf algebras. In addition, there are now some extra features. 
First, in the Hopf setup $J_{H}:=\left(H_{1}\right)$ is in fact a Hopf ideal of $H$. Therefore, $\bar{H}:=H / J_{H}$ is a classical (i.e. super but totally even) commutative Hopf algebra.

Second, the coproduct of $H$ induces also a structure of super left $\bar{H}$-comodule on $H$ (via the projection $H \longrightarrow \bar{H}$ ), such that $H$ is a counital super left $\bar{H}$-comodule $\mathbb{k}$-algebra.

Third, letting $\epsilon: H \longrightarrow \mathbb{k}$ be the counit map of $H$, let $H^{+}:=\operatorname{Ker}(\epsilon), H_{\mathbf{0}}^{+}:=$ $H_{\mathbf{0}} \cap H^{+}, W^{H}:=H_{1} / H_{\mathbf{0}}^{+} H_{1}$ and $\bigwedge W^{H}$ as in Section 3.1.1.

As in Section 3.1.1, $\bar{H} \otimes \wedge W^{H}$ has a natural structure of a commutative superalgebra, endowed with a counit map; moreover, the coproduct of $\bar{H}$ induces on $\bar{H} \otimes \wedge W^{H}$ a super left $\bar{H}$-comodule structure, so that $\bar{H} \otimes \wedge W^{H}$ is a super counital left $\bar{H}$-comodule $\mathbb{k}$-algebra. The notion of a "split" (commutative) Hopf superalgebra - introduced by Masuoka - then reads as follows:

Definition 3.1.8. Any $H \in(\mathrm{H}-\mathrm{salg})_{\mathbb{k}}$ is said to be strongly split if $W^{H}$ is $\mathbb{k}^{-}$ free and there is an isomorphism $\zeta: H \stackrel{\cong}{\longleftrightarrow} \otimes_{\mathbb{k}} \wedge W^{H}$ of super counital left $\bar{H}$-comodule $\mathbb{k}$-algebras.

Remark 3.1.9. The right coadjoint coaction of $H$ canonically induces a right $\bar{H}-$ coaction onto $H$; it is easy to see that this induces an $\bar{H}$-coaction onto $W^{H}$, hence on $\bigwedge W^{H}$ too.

Now assume $H$ is strongly split and $\zeta: H \stackrel{\cong}{\longleftrightarrow} \otimes_{\mathbb{k}} \wedge W^{H}$ is a splitting map as in Definition 3.1.8. We can then endow $\bar{H} \otimes_{\mathbb{k}} \wedge W^{H}$ with the push-forward (via $\zeta$ ) of the coproduct of $H$. Thus $H_{\sigma}:=\bar{H} \otimes_{\mathbb{k}} \wedge W^{H}$ itself is a Hopf superalgebra (isomorphic to $H$ ) such that $\overline{H_{\sigma}} \cong \bar{H}$ and $W^{H_{\sigma}} \cong W^{H}$ in a canonical way. Now, the right coadjoint coaction of $H_{\sigma}$ induces again a right coaction of $\overline{H_{\sigma}}$ onto $\bigwedge W^{H_{\sigma}}$ (as above). It is then immediate to see that — via the identifications $\overline{H_{\sigma}} \cong \bar{H}$ and $\bigwedge W^{H_{\sigma}} \cong \bigwedge W^{H}$ - this coaction is the same as the one of $\bar{H}$ on $\bigwedge W^{H}$.

The following result, due to Masuoka, ensures that $H$ is strongly split (yet he omits the "strong") when the ground ring $\mathbb{k}$ is a field with $\operatorname{char}(\mathbb{k}) \neq 2$ (see also [3], Theorem 1).

Theorem 3.1.10 (cf. [18, Theorem 4.5). If $\mathbb{k}$ is a field with char $(\mathbb{k}) \neq 2$, then each commutative Hopf $\mathbb{k}$-superalgebra is strongly split.

\subsubsection{Examples and counterexamples.}

(a) Consider on $\mathbb{k}[\underline{x}, \underline{\xi}]:=\mathbb{k}\left[x_{1}, \ldots, x_{n}, \xi_{1}, \ldots, \xi_{m}\right] \in(\mathrm{salg})_{\mathbb{k}}$ the standard augmentation given by $\epsilon\left(x_{i}\right):=0, \epsilon\left(\xi_{j}\right):=0$. Then $(\mathbb{k}[\underline{x}, \underline{\xi}], \epsilon) \in(\text { stsp-salg })_{\mathbb{k}}$.

If in addition we consider on $\mathbb{k}[\underline{x}, \underline{\xi}]$ the (standard) Hopf superalgebra structure given by $\Delta\left(x_{i}\right):=x_{i} \otimes 1+1 \otimes x_{i}, \Delta\left(\xi_{j}\right):=\xi_{j} \otimes 1+1 \otimes \xi_{j}, \epsilon\left(x_{i}\right):=0$, $\epsilon\left(\xi_{j}\right):=0, S\left(x_{i}\right):=-x_{i}, S\left(\xi_{j}\right):=-\xi_{j}$, then $\mathbb{k}[\underline{x}, \underline{\xi}]$ is even a strongly split Hopf superalgebra.

(b) For any invertible $u \in \mathbb{k}^{\star}$, let $\mathbb{F}_{2 ; u}:=\mathbb{k}[x, y, \xi, \eta] /(x y+\xi \eta-u)$ with the unique augmentation $\epsilon$ given by $\epsilon(x)=1, \epsilon(y)=u$ and $\epsilon(\xi)=\epsilon(\eta)=0$. Then we have $\overline{\mathbb{F}_{2 ; u}} \cong \mathbb{k}\left[t, t^{-1}\right]$ (via $\bar{x} \mapsto t, \bar{y} \mapsto u t^{-1}$ ), and moreover there exists a section $\sigma_{\mathbb{F}_{2 ; u}}: \overline{\mathbb{F}_{2 ; u}} \longleftrightarrow \mathbb{F}_{2 ; u}$ of the projection $\pi_{\mathbb{F}_{2 ; u}}: \mathbb{F}_{2 ; u} \longrightarrow \overline{\mathbb{F}_{2 ; u}}$ given by $\sigma_{\mathbb{F}_{2 ; u}}(t):=x, \sigma_{\mathbb{F}_{2 ; u}}\left(t^{-1}\right):=u^{-1} y(1+\xi \eta) ;$ thus $\mathbb{F}_{2 ; u} \in(\text { wksp-salg })_{\mathbb{K}}$. Furthermore, we have $\left(\mathbb{F}_{2 ; u}\right)_{\mathbf{1}} \cong \mathbb{k}[\xi, \eta]$ and one easily sees that $\mathbb{F}_{2 ; u} \cong \sigma_{\mathbb{F}_{2 ; u}}\left(\overline{\mathbb{F}_{2 ; u}}\right) \otimes_{\mathbb{k}} \mathbb{k}[\xi, \eta]$ so that $\mathbb{F}_{2 ; u} \in(\text { stsp-salg })_{\mathbb{k}}$. 
(c) Again for any $u \in \mathbb{k}^{\star}$, let $\mathbb{G}_{2 ; u}:=\mathbb{k}[x, y, \xi, \eta, \vartheta, \chi] /(x y+\xi \eta-u, \vartheta \chi)$ with the unique augmentation $\epsilon$ given by $\epsilon(x)=1, \epsilon(y)=u$ and $\epsilon(\xi)=\epsilon(\eta)=$ $\epsilon(\vartheta)=\epsilon(\chi)=0$. Then acting like in (c) one finds that $\mathbb{G}_{2 ; u} \in(\text { spl-salg) })_{\mathbb{k}}$ but $\mathbb{G}_{2 ; u} \notin(\text { stsp-salg })_{\mathbb{k}}$.

(d) Let $\mathbb{F}_{2 ; 0}:=\mathbb{k}[x, y, \xi, \eta] /(x y+\xi \eta)$ with the unique augmentation $\epsilon$ given by $\epsilon(x)=\epsilon(y)=\epsilon(\xi)=\epsilon(\eta)=0$. Again like in (c) one finds easily that $\mathbb{F}_{2 ; 0} \notin(\text { wksp-salg })_{\mathbb{k}}$.

(e) Let $\mathbb{E}_{n, m}:=\mathbb{k}\left[x_{1}, \ldots, x_{n}, \xi_{1}, \ldots, \xi_{m}\right] /\left(\left\{\xi_{j} \xi_{\ell}\right\}_{j, \ell=1, \ldots, m}\right)$ with the obvious augmentation induced by that of $\mathbb{k}[\underline{x}, \underline{\xi}]$ in (a) above. Then $\mathbb{E}_{n, m} \in(\text { cex-salg })_{\mathbb{k}}$.

(f) "Super numbers" on classical algebras: Let $\mathcal{A} \in(\operatorname{alg})_{\mathbb{k}}$ be a commutative $\mathbb{k}$-algebra. If $\eta$ is a formal odd variable, then $\mathcal{A}[\eta]:=\mathcal{A} \otimes_{\mathbb{k}} \mathbb{k}[\eta]$ can naturally be thought of as an object in (salg) ; if we assume $\mathcal{A}$ to have some augmentation, and extend it to $\mathcal{A}[\eta]$ by setting $\epsilon(\eta):=0$, then clearly $\mathcal{A}[\eta]$ is even a(n augmented) central extension. Letting (a-alg) $)_{\mathbb{k}}$ denote the category of commutative $\mathbb{k}$-algebras with augmentation, all this yields a faithful functor $(\mathrm{a}-\mathrm{alg})_{\mathbb{k}} \longrightarrow(\text { cex-salg })_{\mathbb{k}}$.

3.2. Global splittings of superschemes and supergroups. We begin with a general discussion about "global splittings" for affine superschemes; later on we shall look at the case of affine supergroups.

We resume notation as in Section 2.1.1. Let $X \in(\text { assch })_{\mathbb{k}}$ be an affine $\mathbb{k}-$ superscheme, and $R:=\mathcal{O}(X)$ the commutative (unital) $\mathbb{k}$-superalgebra representing it; then let $J_{R}:=\left(R_{\mathbf{1}}\right)=R_{\mathbf{1}}^{[2]} \oplus A_{\mathbf{1}}$ and $\bar{R}:=R / J_{R}=R_{\mathbf{0}} / R_{\mathbf{1}}^{[2]}$.

Definition 3.2.1. The affine superscheme $X_{e v}:=h_{\bar{R}}$ represented by $\bar{R}=\overline{\mathcal{O}(X)}$, i.e., such that $\mathcal{O}\left(X_{e v}\right)=\overline{\mathcal{O}(X)}$, is called the classical scheme associated with $X$. $\diamond$

The terminology just introduced is motivated by the following result:

Proposition 3.2.2. For any $X \in(\text { assch })_{\mathbb{k}}$, we have:

(a) $X_{e v}=X_{\mathbf{0}}$ (see Section 2.1.1), and it can be thought of as a representable functor from $(\mathrm{alg})_{\mathbb{k}}$ to (sets), i.e., as a classical - that is, "non-super" — affine $\mathbb{k}$-scheme;

(b) the $\mathbb{k}$-superscheme $X_{e v}=X_{\mathbf{0}}$ identifies with a closed supersubscheme of $X$; moreover, every closed supersubscheme $X$ which is classical is a closed subscheme of $X_{e v}=X_{\mathbf{0}}$.

Proof. (a) Let $R=\mathcal{O}(X) \in(\operatorname{salg})_{\mathbb{k}}$, so that $X=h_{R}$ and $X_{e v}:=h_{\bar{R}}$. For any $A \in(\mathrm{salg})_{\mathbb{k}}$ we have $X_{e v}(A)=h_{\bar{R}}(A)=\operatorname{Hom}_{\left(\operatorname{salg}_{\mathrm{k}}\right.}(\bar{R}, A)=\operatorname{Hom}_{(\mathrm{alg})_{\mathrm{k}}}\left(\bar{R}, A_{\mathbf{0}}\right)$ because $\bar{R}$ is totally even. In addition, $\operatorname{Hom}_{(\mathrm{alg})_{\mathrm{k}}}\left(\bar{R}, A_{\mathbf{0}}\right)=\operatorname{Hom}_{(\mathrm{salg})_{\mathrm{k}}}\left(R, A_{\mathbf{0}}\right)=$ $h_{R}\left(A_{\mathbf{0}}\right)=X\left(A_{\mathbf{0}}\right)=X_{\mathbf{0}}(A)$, in that, letting $\pi: R \longrightarrow \bar{R}$, for every $\varphi \in$ $\operatorname{Hom}_{(\mathrm{alg})_{\mathrm{k}}}\left(\bar{R}, A_{\mathbf{0}}\right)$ we have $\varphi \circ \pi \in \operatorname{Hom}_{(\mathrm{alg})_{\mathrm{k}}}\left(R, A_{\mathbf{0}}\right)$, and conversely every $\phi \in$ $\operatorname{Hom}_{(\operatorname{alg})_{\mathrm{k}}}\left(R, A_{\mathbf{0}}\right)$ kills $J_{R}$ and thus it factors through $\bar{R}$. Therefore $X_{e v}$ and $X_{\mathbf{0}}$ coincide on objects, and similarly they do on morphisms.

(b) The identification $X_{e v}=X_{\mathbf{0}}$ is a sheer consequence of the (well-known) definition of closed supersubscheme; see for instance [6], §10.1. Moreover, let $Y$ be any (closed) supersubgroup of $X$ which is also classical; then the commutative superalgebra $\mathcal{O}(Y)$ representing $Y$ is a quotient of $R:=\mathcal{O}(G)$, and it is totally even, i.e., it is a (commutative) algebra. Now, any quotient superalgebra of $R$ which 
is totally even is actually a quotient of $\bar{R}=: \mathcal{O}\left(X_{e v}\right)=\mathcal{O}\left(X_{\mathbf{0}}\right)$, by construction. Applying this to $\mathcal{O}(Y)$ we get $Y \subseteq X_{e v}=X_{0}$ as a closed subscheme.

As a consequence of the previous proposition, we shall hereafter denote the classical scheme associated with $X$ by $X_{\mathbf{0}}$ rather than $X_{e v}$.

We can now introduce the notion of "global splitting" for an affine superscheme.

Definition 3.2.3. Let $X \in(\text { assch })_{\mathbb{k}}$ be an affine $\mathbb{k}$-superscheme. We say that $X$ is globally split (or " $g$-split") if there is a superscheme isomorphim $X \cong X_{\mathbf{0}} \times X_{\text {odd }}$ for some totally odd affine $\mathbb{k}$-superscheme $X_{\text {odd }}$ having only one $\mathbb{k}$-point. In addition, we say that $X$ is globally strongly split (or "gs-split") if $X_{\text {odd }}=\mathbb{A}_{\mathbb{k}^{\text {odd }}}$ is indeed a (totally odd) affine $\mathbb{k}$-superspace.

The following algebraic characterization is entirely straightforward:

Proposition 3.2.4. Let $X \in(\text { assch })_{\mathbb{k}}$. Then $X$ is respectively globally split (=" $g$ split") or globally strongly split (= "gs-split") if and only if $\mathcal{O}(X)$ is split or strongly split.

Proposition 3.2 .2 of course applies also to every (affine) supergroup $G$, as a superscheme itself. In addition, we have the following (more or less well-known) result:

Proposition 3.2.5. Let $G$ be an affine supergroup, over a ring $\mathbb{k}$, and let $H:=$ $\mathcal{O}(G)$ be its representing (commutative Hopf) $\mathbb{k}$-superalgebra. Then every closed supersubgroup of $G$ which is classical is a closed subgroup of $G_{\mathbf{0}}=G_{\text {ev }}$.

Proof. If $\mathbf{K}$ is any (closed) supersubgroup of $G$ which is also classical, then the commutative Hopf superalgebra $\mathcal{O}(\mathbf{K})$ representing $\mathbf{K}$ is a quotient of $H:=\mathcal{O}(G)$, and it is totally even, i.e., it is a (commutative) Hopf algebra. Now, any quotient Hopf superalgebra of $H$ which is totally even is actually a quotient of $\bar{H}:=\mathcal{O}\left(G_{\mathbf{0}}\right)$, by construction; applying this to $\mathcal{O}(\mathbf{K})$ we get $\mathbf{K} \leq G_{\mathbf{0}}$.

In light of Masuoka's factorization result for commutative Hopf superalgebras (over fields) in Theorem 3.1.10 the notion of "globally strongly split" (or "gs-split") for supergroups deserves to be made more precise than in the superscheme setting.

Let $G$ be an (affine) $\mathbb{k}$-supergroup, and let $H:=\mathcal{O}(G)$ be the supercommutative Hopf $\mathbb{k}$-superalgebra representing it. The coproduct map $\mathcal{O}(G)=: H \longrightarrow H \otimes H=$ $\mathcal{O}(G) \otimes \mathcal{O}(G)=\mathcal{O}(G \times G)$ corresponds to the multiplication map $G \times G \longrightarrow G$. Similarly, the quotient map $\pi: H:=\mathcal{O}(G) \longrightarrow \mathcal{O}\left(G_{\mathbf{0}}\right)=\bar{H}$ corresponds to a canonical embedding $j: G_{e v}=G_{0} \longleftrightarrow G$. Via this, the $\bar{H}$-coaction

$$
\mathcal{O}(G)=: H \longrightarrow \bar{H} \otimes H=\mathcal{O}\left(G_{\mathbf{0}}\right) \otimes \mathcal{O}(G)=\mathcal{O}\left(G_{\mathbf{0}} \times G\right)
$$

corresponds to a left action $G_{\mathbf{0}} \times G \longrightarrow G$ of $G_{\mathbf{0}}$ onto $G$, given by restriction of the (left) action of $G$ onto $G$ by left multiplication; so $G$ is a left $G_{0}$-scheme. In addition, $G$ has a special point, the unit element - corresponding to the counit map for $H:=\mathcal{O}(G)$ - so that $G$ itself can be thought of as a pointed superscheme.

On the other hand, if $\mathbb{A}_{\mathbb{k}}^{0 \mid d_{-}}$is any totally odd affine $\mathbb{k}^{-}$-superspace (with $d_{-} \in \mathbb{N}$ ), then the direct product $G_{\mathbf{0}} \times \mathbb{A}_{\mathbb{k}}^{0 \mid d_{-}}$has a left $G_{\mathbf{0}^{-}}$action, given by left multiplication in $G_{\mathbf{0}}$; this makes $G_{\mathbf{0}} \times \mathbb{A}_{\mathbb{k}^{0}}^{0 \mid d_{-}}$into a left $G_{\mathbf{0}^{-}}$-scheme. In addition, $G_{\mathbf{0}}$ is also a pointed superscheme, whose special point is the unit element - corresponding to the counit map for $\bar{H}=\mathcal{O}\left(G_{\mathbf{0}}\right)$. But also $\mathbb{A}_{\mathbb{k}}^{0 \mid d_{-}}$is a pointed superscheme, the 
special point being the zero of $\mathbb{A}_{\mathbb{k}^{0}}^{0 \mid d_{-}}$as a free supermodule - this corresponds again to the counit map of the Hopf superalgebra representing $\mathbb{A}_{\mathbb{k}}^{0 \mid d_{-}}$. Therefore, the direct product $\mathbf{G}_{\mathbf{0}} \times \mathbb{A}_{\mathbb{k}}^{0 \mid d_{-}}$is a pointed superscheme as well.

All this leads us to strengthen the notion of "globally split" concerning supergroups.

Definition 3.2.6. Let $G$ be an affine $\mathbb{k}$-supergroup. Assume that there exists a closed subsupercheme $G_{1}$ of $G$ such that

(a) $1_{G} \in G_{\mathbf{1}}$, hence we look at $G_{\mathbf{1}}$ as a pointed superscheme;

(b) the product in $G$ restricts to an isomorphism $G_{0} \times G_{1} \stackrel{\cong}{\rightleftarrows} G$ of pointed left $G_{0}$-superschemes;

(c) $G_{1}$ is (isomorphic to) a totally odd affine superscheme $\mathbb{A}_{\mathbb{k}}^{0 \mid d_{-}}$, as a pointed superscheme.

When all this holds, we say that $G$ is globally strongly split, or in short that it is gs-split.

We shall then denote by (gss-sgroups) $)_{\mathbb{k}}$ and (gss-fsgroups) ${ }_{\mathbb{k}}$, respectively, the full subcategories of (sgroups) $)_{\mathbb{k}}$ and (fsgroups) $)_{\mathbb{k}}$, respectively, whose objects are all supergroups and all fine supergroups, respectively, over $\mathbb{k}$ which in addition are globally strongly split.

Remark 3.2.7. Let $G$ be an affine $\mathbb{k}$-supergroup for which $\operatorname{Lie}(G)$ is quasi-representable. Then if $G$ is globally strongly split, it is also clearly fine - in the sense of Definition 2.3.3

The main facts to take into account at this stage are the following:

Theorem 3.2.8. Let $G$ be an affine supergroup, defined over a ring $\mathbb{k}$, and let $H:=\mathcal{O}(G)$ be its representing (commutative Hopf) $\mathbb{k}$-superalgebra. Then $G$ is globally strongly split if and only if the Hopf superalgebra $\mathcal{O}(G)$ is strongly split. In particular, if $\mathbb{k}$ is a field whose characteristic is not 2 , then $G$ is globally strongly split.

Proof. It is clear that the very last part of the statement is a sheer consequence of Masuoka's Theorem 3.1.10. We just need to prove the rest.

In one direction, the proof is obvious. Indeed, assume that $G$ is globally strongly split, so $G=G_{0} \cdot G_{1} \cong G_{0} \times G_{1} \cong G_{0} \times \mathbb{A}_{\mathbb{k}}^{0 \mid d_{-}}$(see Definition 3.2.6). Then we have isomorphisms

$$
\mathcal{O}(G) \cong \mathcal{O}\left(G_{\mathbf{0}} \times G_{\mathbf{1}}\right) \cong \mathcal{O}\left(G_{\mathbf{0}}\right) \otimes_{\mathbb{k}} \mathcal{O}\left(G_{\mathbf{1}}\right) \cong \mathcal{O}\left(G_{\mathbf{0}}\right) \otimes_{\mathbb{k}} \mathcal{O}\left(\mathbb{A}_{\mathbb{k}}^{0 \mid d_{-}}\right)
$$

of $\mathbb{k}_{\mathrm{k}}$-algebras. Moreover, the fact that $G=G_{\mathbf{0}} \cdot G_{\mathbf{1}} \cong G_{\mathbf{0}} \times G_{\mathbf{1}}$ as pointed superschemes implies that the resulting isomorphism $\mathcal{O}(G) \cong \mathcal{O}\left(G_{\mathbf{0}}\right) \otimes_{\mathbb{k}} \mathcal{O}\left(\mathbb{A}_{\mathbb{k}}^{0 \mid d_{-}}\right)$ is also counital. Finally, as the isomorphism $G_{\mathbf{0}} \times \mathbb{A}_{\mathrm{k}}^{0 \mid d_{-}} \cong G_{\mathbf{0}} \cdot G_{\mathbf{1}}=G$ is one of $G_{\mathbf{0}}$-superspaces, one sees in addition that the isomorphism $\mathcal{O}(G) \cong \mathcal{O}\left(G_{\mathbf{0}}\right) \otimes_{\mathbb{k}}$ $\mathcal{O}\left(\mathbb{A}_{\mathbb{k}}^{0 \mid d_{-}}\right)$is also left $\mathcal{O}\left(G_{\mathbf{0}}\right)$-coinvariant.

The converse step almost entirely follows from definitions (along with Remark 3.1.9) and Masuoka's result (Theorem 3.1.10). Indeed, assume that the Hopf superalgebra $H:=\mathcal{O}(G)$ is strongly split, i.e., there exists an isomorphism $\mathcal{O}(G)=$ : $H \stackrel{\cong}{\longrightarrow} \otimes_{\mathbb{k}} \wedge W^{H}$ of counital $\bar{H}$-comodule superalgebras, with $\bar{H}=\overline{\mathcal{O}(G)}=$ $\mathcal{O}\left(G_{0}\right)$. Using this isomorphism we can identify both $\bar{H}=\overline{\mathcal{O}(G)}=\mathcal{O}\left(G_{0}\right)$ and 
$\bigwedge W^{H}$ with subalgebras of $\mathcal{O}(G)=H$ whose product is all of $H$ itself. Taking superspectra, this yields an isomorphism $G_{0} \times G_{1} \cong G$ - as pointed superschemes with left $G_{\mathbf{0}}$-action - for some closed subsuperscheme $G_{\mathbf{1}}$ in $G$ such that $\mathcal{O}\left(G_{\mathbf{1}}\right)=\bigwedge W^{H}$. To put it in down-to-earth terms, we look pointwise: if $A \in(\mathrm{salg})_{\mathbb{k}}$ one has

$$
\begin{gathered}
G(A):=\operatorname{Hom}_{\left(\mathrm{salg}_{\mathrm{k}}\right.}(H, A), \quad G_{\mathbf{0}}(A):=\operatorname{Hom}_{(\mathrm{salg})_{\mathrm{k}}}(\bar{H}, A) \\
G_{\mathbf{1}}(A):=\operatorname{Hom}_{(\mathrm{salg})_{\mathrm{k}}}\left(\bigwedge W^{H}, A\right)
\end{gathered}
$$

and the isomorphism then is given by $\left(m_{A}\right.$ being the multiplication in $A$ )

$$
G_{\mathbf{0}}(A) \times G_{\mathbf{1}}(A) \longrightarrow G(A), \quad\left(\varphi_{0}, \varphi_{1}\right) \mapsto m_{A} \circ\left(\varphi_{0} \otimes \varphi_{1}\right) .
$$

In particular, $G_{\mathbf{0}}(A)$ and $G_{\mathbf{1}}(A)$ as subsets of $G(A)$ are characterized as

$$
\begin{gathered}
G_{\mathbf{0}}(A):=\left\{\phi \in G(A) \mid \phi(1 \otimes \omega)=\epsilon_{\wedge W^{H}}(\omega) \forall \omega \in \wedge W^{H}\right\}, \\
G_{\mathbf{1}}(A):=\left\{\phi \in G(A) \mid \phi(\bar{h} \otimes 1)=\epsilon_{\bar{H}}(\bar{h}) \forall \bar{h} \in \bar{H}\right\} .
\end{gathered}
$$

Thus, the only non-trivial point which is left out is that this isomorphism actually is realized through restriction of the product in $G$ and we now check it pointwise.

The standard embedding $\sigma_{H}: \mathcal{O}\left(G_{0}\right)=\overline{\mathcal{O}(G)}=\bar{H} \longleftrightarrow \bar{H} \otimes_{\mathbb{k}} \wedge W^{H} \cong H=$ : $\mathcal{O}(G)$ induces a natural supergroup morphism $G \longrightarrow G_{\mathbf{0}}$, which is a retraction of the embedding $G_{0} \longleftrightarrow G$ because $\sigma_{H}$ itself is a section of the canonical projection $\pi_{H}: H \longrightarrow \bar{H}$. Putting it in down-to-earth terms, for $A \in(\mathrm{salg})_{\mathbb{k}}$ the abovementioned retraction reads

$G(A):=\operatorname{Hom}_{(\mathrm{salg})_{\mathbf{k}}}(\mathcal{O}(G), A) \rightarrow G_{\mathbf{0}}(A):=\operatorname{Hom}_{(\mathrm{salg})_{\mathbf{k}}}\left(\mathcal{O}\left(G_{\mathbf{0}}\right), A\right), \quad g \mapsto g_{0}:=g \circ \sigma$ for all $g \in G(A)$. In other words, if $g \in G(A)$, then $g_{0}:=g \circ \sigma$ can be seen as the restriction of $g$ (which is a morphism from $H:=\mathcal{O}(G)$ to $A$ ) to $\bar{H}=\overline{\mathcal{O}(G)}$ embedded in $H=\mathcal{O}(G)$ via $\sigma$. Notice also that $g_{0}:=g \circ \sigma$ belongs to $G_{0}(A)$, and when we embed the latter (canonically) into $G(A)$ the image of $g_{0}$ is $g_{0} \circ \pi_{H}$. In other words, when thinking of $g_{0}$ as an element of $G(A)$ we realize that we are actually taking $\hat{g}_{0}:=g_{0} \circ \pi_{H}$.

Now for each $g \in G(A)$ consider $g_{0}:=g \circ \sigma \in G_{\mathbf{0}}(A)$ and $\hat{g}_{0}:=g_{0} \circ \pi_{H} \in$ $G(A)$ as above; then define $g_{1}:=\hat{g}_{0}^{-1} \cdot g(\in G(A))$. By construction we have $g=\hat{g}_{0} \cdot g_{1}$ - a product inside $G(A)$ - so we are only left to prove that actually $g_{1} \in G_{\mathbf{1}}(A)=\operatorname{Hom}_{(\operatorname{salg})_{\mathrm{k}}}\left(\bigwedge W^{H}, A\right)$. To this end, we recall that the product in $G(A)=\operatorname{Hom}_{(\operatorname{salg})_{\mathrm{k}}}(H, A)$ is given by convolution

$$
g^{\prime} \cdot g^{\prime \prime}:=m_{A} \circ\left(g^{\prime} \otimes g^{\prime \prime}\right) \circ \Delta_{H} \quad \forall g^{\prime}, g^{\prime \prime} \in G(A)=H_{(\operatorname{salg})_{\mathbf{k}}}(H, A) .
$$

By the characterization of $G_{\mathbf{1}}(A)$ given above we have that $g_{1} \in G_{\mathbf{1}}(A)$ if and only if

$$
g_{1}(\bar{h} \otimes 1)=1_{G(A)}(\bar{h} \otimes 1) \quad \text { for all } \quad \bar{h} \in \bar{H}
$$

so this is our goal. Definitions along with (3.1) give

$$
g_{1}:=\hat{g}_{0}^{-1} \cdot g=m_{A} \circ\left(\hat{g}_{0}^{-1} \otimes g\right) \circ \Delta_{H}=m_{A} \circ\left(g_{0}^{-1} \otimes g\right) \circ\left(\pi_{H} \otimes i d_{H}\right) \circ \Delta_{H} .
$$

Recall that, by assumption, the isomorphism $H \stackrel{\cong}{\leftrightarrows} \otimes_{\mathbb{k}} \wedge W^{H}$ of counital superalgebras is also left $\bar{H}$-covariant. This means that

$$
\left(\left(\pi_{H} \otimes i d_{H}\right) \circ \Delta_{H}\right)(\bar{h} \otimes 1)=\left(\Delta_{\bar{H}} \otimes i_{\wedge^{H}}\right)(\bar{h} \otimes 1)=\bar{h}_{(1)} \otimes \bar{h}_{(2)} \otimes 1 \quad \forall \bar{h} \in \bar{H}
$$


hence (3.3) and the identity $\bar{h}_{(2)} \otimes 1=\sigma\left(\bar{h}_{(2)}\right)$ together give

$$
\begin{array}{r}
g_{1}(\bar{h} \otimes 1)=\left(m_{A} \circ\left(g_{0}^{-1} \otimes g\right) \circ\left(\pi_{H} \otimes i d_{H}\right) \circ \Delta_{H}\right)(\bar{h} \otimes 1) \\
=g_{0}^{-1}\left(\bar{h}_{(1)}\right) g\left(\bar{h}_{(2)} \otimes 1\right)=g_{0}^{-1}\left(\bar{h}_{(1)}\right)(g \circ \sigma)\left(\bar{h}_{(2)}\right)=g_{0}^{-1}\left(\bar{h}_{(1)}\right) g_{0}\left(\bar{h}_{(2)}\right) \\
=\left(g_{0}^{-1} \cdot g_{0}\right)(\bar{h})=1_{G_{\mathbf{O}}(A)}(\bar{h})=1_{G(A)}(\bar{h} \otimes 1)
\end{array}
$$

for all $\bar{h} \in \bar{H}$, so that (3.2) is proved.

The above characterization of gs-split supergroups yields an interesting consequence.

Corollary 3.2.9. Let $G$ be a globally strongly split supergroup. Then (with notation of Definition 3.2.6) $G_{\mathbf{1}}$ is stable by the adjoint $G_{\mathbf{0}}$-action.

Proof. Applying Remark 3.1.9 to $H:=\mathcal{O}(G)$ we find that $\bigwedge W^{H}=\mathcal{O}\left(G_{\mathbf{1}}\right)$ is stable by the right coadjoint $\mathcal{O}\left(G_{\mathbf{0}}\right)$-coaction. But at the superscheme level this implies exactly that $G_{\mathbf{1}}$ is stable by the adjoint $G_{\mathbf{0}}$-action.

Remark 3.2.10. To be complete, we mention that in Boseck's approach (see [3,5]) each affine algebraic supergroup is assumed to be "globally strongly split" (in our sense) by definition.

3.2.11. Consistent splittings for embeddings of gs-split supergroups. If we consider a (closed) supersubgroup within a supergroup, and both are gs-split, we can ask whether there exist "splittings" of both supergroups which are "consistent" with each other, by which we mean that they are "compatible", in some natural sense, with the embedding of the first supergroup inside the second one. We shall now make this rough idea more precise and find some significant results.

Let us start with an affine, fine supergroup $G$ over $\mathbb{k}$, with $\mathfrak{g}=\operatorname{Lie}(G)$. Set $A:=$ $\mathcal{O}(G), \bar{A}:=A /\left(A_{1}\right), W^{A}:=A_{1} / A_{0}^{+} A_{1}$. Recall that $\bar{A}=\overline{\mathcal{O}(G)}=\mathcal{O}\left(G_{e v}\right)$, the commutative Hopf algebra which represents the affine group $G_{e v}$ associated with $G$, and $\left(W^{A}\right)^{*}=\mathfrak{g}_{1}$. By assumption $G$ is fine, so $\mathfrak{g}_{1}$ is $\mathbb{k}$-finite free. Recall also that $\mathfrak{g}$ is endowed with a $2-$ operation and the universal enveloping (super)algebra $U(\mathfrak{g})$ involves the relations $v^{2}=v^{\langle 2\rangle}$, for $v \in \mathfrak{g}_{1}$. Just as in [20], Lemma 4.24, there exists a canonical Hopf pairing $\langle\rangle:, U(\mathfrak{g}) \times A \longrightarrow \mathbb{k}$ which gives rise to the superalgebra map $\kappa: A \longrightarrow U(\mathfrak{g})^{*}$ defined by $\kappa(a):=\langle, a\rangle$.

Choose a totally ordered $\mathbb{k}$-free basis $X$ of $\mathfrak{g}_{1}$, and define a unit-preserving supercoalgebra map $\iota_{X}: \wedge \mathfrak{g}_{1} \longrightarrow U(\mathfrak{g})$ by $\iota_{X}\left(x_{1} \wedge \cdots \wedge x_{n}\right):=x_{1} \cdots x_{n}$ for $n \geq 0$ and $x_{1}<\cdots<x_{n}$ in $X$. Define also $\rho_{X}: A \longrightarrow \wedge W^{A}$ to be the composition $\rho_{X}: A \stackrel{\kappa}{\longrightarrow} U(\mathfrak{g})^{*} \stackrel{\iota_{X}^{*}}{\longrightarrow}\left(\bigwedge \mathfrak{g}_{1}\right)^{*} \stackrel{\cong}{\longrightarrow} \bigwedge W^{A}$ where the last arrow denotes the canonical isomorphism; this $\rho_{X}$ is a counit-preserving superalgebra map; see [18], page 301. Now, assume that the Hopf superalgebra $A$ is split (or equivalently $G$ is gs-split). Thus there exists a counit-preserving isomorphism $A \stackrel{\cong}{\longrightarrow} \otimes_{\mathbb{k}} \wedge W^{A}$ of left $A$-comodule superalgebras. The following result proves that one can choose a particular such splitting:

Lemma 3.2.12 (Masuoka). The map $\psi_{X}: A \longrightarrow \bar{A} \otimes_{\mathbb{k}} \wedge W^{A}, a \mapsto \psi_{X}(a):=$ $\overline{a_{(1)}} \otimes \rho_{X}\left(a_{(2)}\right)$, where $a \mapsto \bar{a}$ denotes the natural projection $A \longrightarrow \bar{A}$, is bijective. In fact, it is a counit-preserving isomorphism of left $\bar{A}$-comodule superalgebras. 
Proof. The claim follows from [20], Lemma 4.27; note in particular that the cited lemma actually holds over an arbitrary ring $\mathbb{k}$ (moreover, $\bar{A}$ may not be finitely generated, as long as $W^{A}$ is $\mathbb{k}$-finite free; see the proof of Theorem A.10 in 20]). Since the map $\rho_{X}$, composed with the natural projection $\wedge W^{A} \longrightarrow W^{A}$, coincides with the natural composite

$$
A=\mathbb{k} \oplus A^{+} \longrightarrow A^{+} /\left(A^{+}\right)^{2} \longrightarrow\left(A^{+} /\left(A^{+}\right)^{2}\right)_{1}=W^{A}
$$

we conclude that $\rho_{X}$ satisfies the assumption required by the cited lemma.

We can now state and prove the promised result about the existence of "consistent" splittings for a closed embedding between gs-split supergroups.

Theorem 3.2.13 (Gavarini and Masuoka). Let $H$ and $K$ be (affine, fine) supergroups over $\mathbb{k}$, with $H$ being a closed subsupergroup of $K$. Setting $\mathfrak{h}:=\operatorname{Lie}(H)$, $\mathfrak{k}:=\operatorname{Lie}(K)$, assume that:

(a) the quotient $\mathbb{k}$-supermodule $\mathfrak{k}_{\mathbf{1}} / \mathfrak{h}_{\mathbf{1}}$ is free;

(b) $H$ and $K$ are globally strongly split.

Then, for a given splitting $H=H_{0} \times H_{1}$ of $H$, there exists a similar splitting $K=K_{\mathbf{0}} \times K_{\mathbf{1}}$ of $K$ which is consistent with that of $H$, that is, one has $H_{\mathbf{0}} \subseteq K_{\mathbf{0}}$, $H_{1} \subseteq K_{1}$ and the diagram

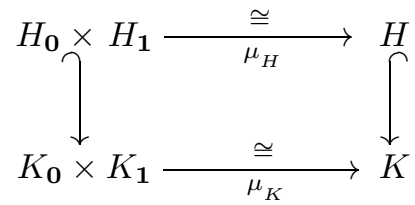

( $\mu_{H}$ being an isomorphism of pointed affine $H_{0}$-superschemes, and similarly $\mu_{K}$ ) is commutative.

Proof. Set $A:=\mathcal{O}(K)$ and $B:=\mathcal{O}(H)$. By assumption $\mathfrak{k}_{\mathbf{1}}$ and $\mathfrak{h}_{\mathbf{1}}$ are $\mathbb{k}$-finite free, so the same holds true for their linear dual $A_{1} / A_{0}^{+} A_{1}=\mathfrak{k}_{1}^{*}$ and $B_{1} / B_{0}^{+} B_{1}=\mathfrak{h}_{1}^{*}$. Moreover, (a) ensures that there exists a free $\mathbb{k}$-subsupermodule $\mathfrak{q}$ of $\mathfrak{k}_{\mathbf{1}}$ such that $\mathfrak{k}_{1}=\mathfrak{h}_{1} \oplus \mathfrak{q}$. Finally, let $K_{1}$ and $H_{1}$ be the pointed (affine algebraic) superschemes represented by $\Lambda\left(\mathfrak{g}_{1}^{*}\right)$ and $\Lambda\left(\mathfrak{h}_{1}^{*}\right)$ respectively. Since $H$ is a closed subsupergroup of $K$, it follows that $H_{0}=H_{e v}$ can be seen as a closed (classical) subgroup of $K_{\mathbf{0}}=K_{e v}$; similarly, $H_{\mathbf{1}}$ can be seen as a closed pointed subsuperscheme of $K_{\mathbf{1}}$.

If $X_{\mathfrak{k}}$ is an ordered basis of $\mathfrak{k}_{1}$ and $X_{\mathfrak{h}}$ is one of $\mathfrak{h}_{1}$ we can construct splitting isomorphisms $\psi_{X_{\mathfrak{k}}}$ and $\psi_{X_{\mathfrak{h}}}$ as in Lemma 3.2 .12 for $A^{\prime}:=\mathcal{O}(K)$ and $A^{\prime \prime}:=\mathcal{O}(H)$ respectively. Then moving backwards from Hopf superalgebras to supergroups we find splitting maps $\mu_{K}: K_{\mathbf{0}} \times K_{\mathbf{1}} \stackrel{\cong}{\longrightarrow} K$ and $\mu_{H}: H_{\mathbf{0}} \times H_{1} \cong H$ as in Section 3.2 .11 for $H$ and $K$, with $\mathcal{O}\left(\mu_{K}\right)=\psi_{X_{\mathfrak{k}}}, \mathcal{O}\left(\mu_{H}\right)=\psi_{X_{\mathfrak{h}}}$.

Now, by assumption the natural injection $\mathfrak{h}_{1} \longrightarrow \mathfrak{k}_{1}$ is $\mathbb{k}$-linearly split, as $\mathfrak{k}_{1}=$ $\mathfrak{h}_{\mathbf{1}} \oplus \mathfrak{q} ;$ moreover, $\mathfrak{q}$ is $\mathbb{k}$-free and therefore we can choose $X_{\mathfrak{h}}$ and $X_{\mathfrak{k}}$ as above so that the former is an ordered subset of the latter. For such a choice, the diagram

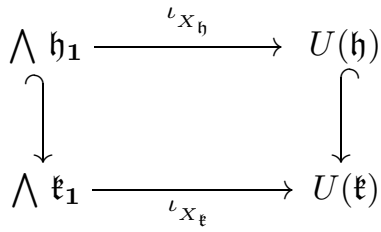


is commutative, which in turn implies that the diagram

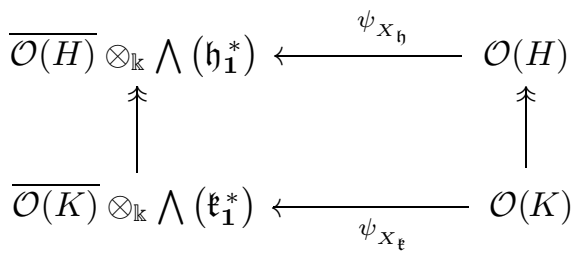

is commutative too. When turning back from superalgebras to superschemes, this implies that the diagram in the statement is commutative as well.

Remark 3.2.14. The assumption (a) in Theorem 3.2.13 obviously holds true when $\mathbb{k}$ is a PID, or - more generally — whenever every finitely generated projective $\mathbb{k}$-module is free.

3.3. Splittings on $A$-points. In this subsection we dwell upon some special splittings of supergroups which arise when we take their $A$-points for some special superalgebras $A$, i.e., when we restrict them - as functors - on special subcategories of $(\operatorname{salg})_{\mathbb{k}}$.

Definition 3.3.1. Let $G:(\mathrm{salg})_{\mathbb{k}} \longrightarrow$ (sgroups) be a supergroup $\mathbb{k}$-functor. Then there exists a unique, well-defined normal subgroup $\mathbb{k}$-functor of $G$, denoted $\operatorname{Ker}(\pi)_{G}$ and given on objects by $\operatorname{Ker}(\pi)_{G}(A):=\operatorname{Ker}\left(G\left(\pi_{A}\right)\right)$ for every $A \in(\text { wksp-salg })_{\mathbb{K}}$.

In general, in the study of a supergroup functor $G$ the normal subgroup functor $\operatorname{Ker}(\pi)_{G}$ is not of great use. But restricting to weakly split superalgebras, the next result shows that it splits into a semidirect product, in which $\operatorname{Ker}(\pi)_{G}$ is the normal factor.

Proposition 3.3.2. Let $G$ be a $\mathbb{k}$-supergroup functor and $A \in(\text { wksp-salg })_{\mathbb{k}}$.

(a) The group $G(A)$ splits into a semidirect product

$$
G(A)=\bar{G}(A) \ltimes \operatorname{Ker}(\pi)_{G}(A)
$$

with (see Definition 2.1.6) $\bar{G}(A):=G(\bar{A})$ and $\operatorname{Ker}(\pi)_{G}(A):=\operatorname{Ker}\left(G\left(\pi_{A}\right)\right)$. Therefore, denoting by $\dot{F}$ the restriction to (wksp-salg) $)_{\mathbb{k}}$ of any superfunctor $F$, we have that the functor $\dot{G}:$ (wksp-salg) $)_{\mathbb{k}} \longrightarrow$ (groups) splits into a semidirect product

$$
\dot{G}=\dot{\bar{G}} \ltimes \operatorname{Ker}(\pi)_{G} .
$$

(b) The group $G_{\mathbf{0}}(A)$ splits into a semidirect product

$$
G_{\mathbf{0}}(A)=\bar{G}(A) \ltimes \operatorname{Ker}(\pi)_{G_{\mathbf{0}}}(A)
$$

with (see Definition 2.1.6) $\operatorname{Ker}(\pi)_{G, \mathbf{0}}(A):=\operatorname{Ker}(\pi)_{G}(A) \cap G_{\mathbf{0}}(A)$. Thus the $\mathbb{k}^{-}$ supergroup functor $\dot{G}_{\mathbf{0}}:$ (wksp-salg) $)_{\mathbb{k}} \longrightarrow$ (groups) splits into a semidirect product

$$
\dot{G}_{0}=\dot{\bar{G}} \ltimes \operatorname{Ker}(\pi)_{G_{0}} .
$$

Proof. As $A \in(\text { wksp-salg })_{\mathbb{k}}$, we have a projection $\pi_{A}: A \longrightarrow \bar{A}$ with section $\sigma_{A}: \bar{A} \longleftrightarrow A$ within $(\mathrm{salg})_{\mathbb{k}}$. Applying $G$ we get that $G\left(\sigma_{A}\right): G(\bar{A}) \longrightarrow G(A)$ is a section of $G\left(\pi_{A}\right): G(A) \longrightarrow G(\bar{A})$, so $G\left(\sigma_{A}\right)$ is a monomorphism and $G\left(\pi_{A}\right)$ an epimorphism. In turn, this yields then a semidirect product factorization of 
$G(A)$, namely $G(A)=\operatorname{Im}\left(G\left(\sigma_{A}\right)\right) \ltimes \operatorname{Ker}\left(G\left(\pi_{A}\right)\right)$. Looking at definitions one finds $\operatorname{Im}\left(G\left(\sigma_{A}\right)\right) \cong G(\bar{A})$ whence claim (a) follows.

As to claim (b), one can repeat the previous argument; just replace $G$ with $G_{\mathbf{0}}$ - such that $A \mapsto G\left(A_{\mathbf{0}}\right)$ - and $\operatorname{Ker}(\pi)_{G}$ with $\operatorname{Ker}(\pi)_{G_{\mathbf{0}}}$ wherever they occur.

The previous result reads better when applied to split superalgebras.

Proposition 3.3.3. Let $G$ be a $\mathbb{k}$-supergroup functor and $A \in(\mathrm{spl}-\mathrm{salg})_{\mathbb{k}}$.

(a) The group $G(A)$ splits into a semidirect product

$$
G(A)=\bar{G}(A) \ltimes G_{1}^{(1)}(A)
$$

with (see Definition 2.1.6) $\bar{G}(A):=G(\bar{A})$ and $G_{1}^{(1)}(A):=G\left(A_{1}^{(1)}\right)$. Therefore, denoting by $\hat{F}$ the restriction to (spl-salg) $)_{\mathbb{k}}$ of any superfunctor $F$, we have that $\hat{G}:(\text { spl-salg })_{\mathbb{k}} \longrightarrow$ (groups) splits into a semidirect product

$$
\hat{G}=\hat{\bar{G}} \ltimes \hat{G}_{1}^{(1)} .
$$

(b) The group $G_{\mathbf{0}}(A)$ splits into a semidirect product

$$
G_{\mathbf{0}}(A)=\bar{G}(A) \ltimes G_{\mathbf{1}}^{(2)}(A)
$$

with (see Definition 2.1.6) $G_{\mathbf{1}}^{(2)}(A):=G\left(A_{1}^{(2)}\right)$. Thus, with notation as in $(a)$, $\hat{G}_{\mathbf{0}}:(\mathrm{spl}-\mathrm{salg})_{\mathbb{k}} \longrightarrow$ (groups) splits into a semidirect product

$$
\hat{G}_{\mathbf{0}}=\hat{\bar{G}} \ltimes \hat{G}_{\mathbf{1}}^{(2)} .
$$

Proof. From the natural embedding $A_{1}^{(1)} \longleftrightarrow \bar{A} \otimes_{\mathbb{k}} A_{1}^{(1)}=A$ in (wksp-salg) $)_{\mathbb{k}}$ we get a group morphism $G_{1}^{(1)}(A):=G\left(A_{1}^{(1)}\right) \longrightarrow G(A)$. Directly from definitions, one finds that the latter too is an embedding and moreover $\operatorname{Ker}(\pi)_{G}(A)=G_{1}^{(1)}(A)$ for $A \in(\mathrm{spl}-\mathrm{salg})_{\mathbb{k}}$; then claims (a) and (b) follow from this and Proposition 3.3 .2 right above.

The next result still improves the previous one when we restrict to central extension algebras.

Proposition 3.3.4. Let notation be as in Proposition 3.3 .2 . Let $G \in(\text { sgroups) })_{\mathbb{k}}$ be $a \mathbb{k}$-supergroup and $A \in(\text { cex-salg })_{\mathbb{k}}$. Then $G(A)$ splits into a semidirect product $G(A)=\bar{G}(A) \ltimes G_{\mathbf{1}}^{(1)}(A)$ with $\bar{G}(A)=G_{\mathbf{0}}(A)$. Thus, letting $\check{F}$ be the restriction to $(\text { cex-salg })_{\mathbb{k}}$ of any superfunctor $F$, we have that $\check{G}:(\text { cex-salg })_{\mathbb{k}} \longrightarrow$ (groups) splits into a semidirect product $\check{G}=\bar{G} \ltimes \check{G}_{\mathbf{1}}^{(1)}=\check{G}_{\mathbf{0}} \ltimes \check{G}_{\mathbf{1}}^{(1)}$.

Proof. As $A \in(\text { cex-salg })_{\mathbb{k}}$ we have $\bar{A}=A_{\mathbf{0}}$, whence everything follows.

\subsection{Examples and applications.}

We provide some examples to illustrate the previously explained ideas. Besides their intrinsic interest, these will also be useful in the sequel.

3.4.1. Supergroups on "super-numbers" as (classical) groups of "super-points". Let $\mathcal{A} \in(\mathrm{alg})_{\mathbb{k}}$ be a commutative $\mathbb{k}$-algebra. Like in Example $3.1 .11(c)$, we consider the associated central extension $\mathcal{A}[\eta]=\mathcal{A} \oplus \mathcal{A} \eta \in(\text { cex-salg })_{\mathbb{k}}$. Loosely inspired by the similar construction of "dual numbers" - either in the non-super or the super framework - we call its elements "super $\mathcal{A}$-numbers", thinking of those in $\mathcal{A}$ itself as "even super-numbers" and those in $\mathcal{A} \eta$ as "odd super-numbers". Now, 
as $\mathcal{A}[\eta] \in(\text { cex-salg })_{\mathbb{k}}$ we have $(\mathcal{A}[\eta])_{\mathbf{0}}=\mathcal{A},(\mathcal{A}[\eta])_{\mathbf{1}}^{(1)}=\mathbb{k} \oplus \mathcal{A} \eta$. Then for any $G \in(\text { sgroups })_{\mathbb{k}}$ Proposition 3.3 .4 above yields

$$
G(\mathcal{A}[\eta])=G_{\mathbf{0}}(\mathcal{A}) \ltimes G_{\mathbf{1}}^{(1)}(\mathbb{k} \oplus \mathcal{A} \eta) .
$$

Now, the left hand factor $G_{\mathbf{0}}(\mathcal{A})$ of $G(\mathcal{A}[\eta])$ is the group of $\mathcal{A}$-points of the classical (= non-super) affine group-scheme $G_{\mathbf{0}}$, hence its elements are nothing but classical (= non-super) points of a classical group-scheme. For this reason, we suggest we think of these as being "even $\mathcal{A}$-superpoints" of $G$, and similarly to think of the elements of the right hand factor $G_{1}^{(1)}(\mathbb{k} \oplus \mathcal{A} \eta)$ of $G(\mathcal{A}[\eta])$ as being "odd $\mathcal{A}$ superpoints" of $G$.

Now assume in addition that $G$ is strongly split, say $G=G_{e v} \times G_{o d d}=G_{\mathbf{0}} \times G_{\text {odd }}$ with $G_{\text {odd }} \cong \mathbb{A}_{\mathrm{k}}^{\text {odd }}$ (notation of Subsection 3.2). Then we have

$$
G_{\mathbf{1}}^{(1)}(\mathbb{k} \oplus \mathcal{A} \eta)=G_{\text {odd }}(\mathbb{k} \oplus \mathcal{A} \eta)=\mathbb{A}_{\mathbb{k}}^{\text {odd }}(\mathbb{k} \oplus \mathcal{A} \eta)=\mathcal{A}^{0 \mid I}=\mathcal{A}^{I}
$$

where $0 \mid I$ is the (possibly infinite) super-dimension of $\mathbb{A}_{\mathbb{k}}^{\text {odd }}$ and $\mathcal{A}^{I}$ is taken with odd parity. Thus $G_{\mathbf{1}}^{(1)}(\mathbb{k} \oplus \mathcal{A} \eta)=\mathcal{A}^{I}$ identifies with the set of $\mathcal{A}$-points of the classical (= totally even, or non-super) affine scheme $\mathcal{A}^{I} \equiv \mathcal{A}^{I \mid 0}$. Therefore, by (3.4) and (3.5) together we conclude that computing the $\mathbb{k}$-superscheme $G$ on the central extensions given by "super-numbers" on classical algebras — e.g., on $\mathcal{A}[\eta]$, say — is the same as computing the (classical!) $\mathbb{k}$-scheme $G_{\mathbf{0}} \times \mathbb{A}_{\mathbb{k}}^{I}$ on classical algebras - namely on $\mathcal{A}$, say.

3.4.2. Splittings on "Grassmann-points". Let $G$ be a $\mathbb{k}$-supergroup, and $\Lambda=$ $\mathbb{k}\left[\left\{\xi_{i}\right\}_{i \in I}\right]$ any Grassmann algebra, possibly infinite-dimensional. Obviously $\Lambda \in$ $(\text { stsp-salg })_{\mathbb{k}}$ - for a unique, canonical augmentation - hence we have a splitting of the group $G(\Lambda)$ of $\Lambda$-points of $G$ as in Proposition 3.3.3 (a). In particular, this is exactly the splitting mentioned by Boseck in [3], $\S 2$, where indeed only $\Lambda$-points of supergroups are considered.

3.4.3. Global splittings of general linear supergroups. Let $\mathrm{GL}(V)$ be a linear supergroup as in Example 2.1.5(b), defined over some ground ring $\mathbb{k}$. Letting $p \mid q:=$ $r k\left(V_{\mathbf{0}}\right) \mid r k\left(V_{\mathbf{1}}\right)$ be the (finite, by assumption) super-dimension of $V$, we shall also write $\mathrm{GL}_{p \mid q}:=\mathrm{GL}(V)$. In particular, this means that each element of $\mathrm{GL}_{p \mid q}(A):=(\operatorname{GL}(V))(A)$ can be written as a block matrix $\left(\frac{a \mid \beta}{\gamma \mid d}\right)$ where $a$, $\beta, \gamma, d$ are matrices of size $p \times p, p \times q, q \times p, q \times q$ respectively, and whose entries respectively belong to $A_{0}, A_{1}, A_{1}$ and $A_{0}$.

The condition that such a block matrix in $\mathfrak{g l}_{p \mid q}(A)$ belong to $\mathrm{GL}_{p \mid q}(A)$ - i.e., that it be invertible - amounts to $a$ and $d$ being invertible on their own (see [6], $\S 1.5)$.

Note also that $\left(\mathrm{GL}_{p \mid q}\right)_{e v}(A)$ has a neat description: it is the subgroup of all those block matrices for which (in the previous notation) $\beta=0=\gamma$.

We shall now show that $\mathrm{GL}(V)$ is strongly split. Note that this does not depend on the nature of the ground ring $\mathbb{k}$ - in particular, we do not need it to be a field.

Define $\left(\mathrm{GL}_{p \mid q}\right)_{\text {odd }}:=I+\left(\mathfrak{g l}_{p \mid q}\right)_{1}$, where $I:=I_{p+q}$ is the identity (block) matrix of size $(p+q) \times(p+q)$. This is clearly a totally odd affine superspace, which is stable by the adjoint action of $\left(\mathrm{GL}_{p \mid q}\right)_{e v}$ — both being considered embedded inside 
$\mathrm{GL}_{p \mid q}$. Now, a direct check shows that any $\left(\frac{a \mid \beta}{\gamma \mid d}\right) \in \mathrm{GL}_{p \mid q}(A)$ admits a unique factorization, w.r.t. the matrix product, as

$$
\left(\frac{a \mid \beta}{\gamma \mid d}\right)=\left(\frac{a \mid 0}{0 \mid d}\right) \cdot\left(\frac{I_{p} \mid a^{-1} \beta}{d^{-1} \gamma \mid I_{q}}\right)
$$

This provides a map $\mathrm{GL}_{p \mid q}(A) \longrightarrow\left(\mathrm{GL}_{p \mid q}\right)_{e v}(A) \times\left(\mathrm{GL}_{p \mid q}\right)_{\text {odd }}(A)$ which, for $A$ ranging in $(\mathrm{salg})_{\mathbb{k}}$, eventually provides a global splitting as we were looking for.

Instead of the above geometric approach, one can follow an algebraic one. For that, one simply has to notice that

$$
\begin{aligned}
& \mathcal{O}\left(\mathrm{GL}_{p \mid q}\right)=\mathbb{k}\left[\left\{x_{i, j}^{\prime}, x_{h, k}^{\prime \prime}, \xi_{i, k}^{\prime}, \xi_{h, j}^{\prime \prime}\right\}_{i, j=1, \ldots, p ;}^{h, k=1, \ldots, q}, \operatorname{det}\left(X^{\prime}\right)^{-1}, \operatorname{det}\left(X^{\prime \prime}\right)^{-1}\right] \\
& =\mathbb{k}\left[\left\{x_{i, j}^{\prime}, x_{h, k}^{\prime \prime}\right\}_{i, j=1, \ldots, p ;}^{h, k=1, \ldots, q ;}, \operatorname{det}\left(X^{\prime}\right)^{-1}, \operatorname{det}\left(X^{\prime \prime}\right)^{-1}\right] \otimes_{\mathbb{k}} \mathbb{k}\left[\left\{\xi_{i, k}^{\prime}, \xi_{h, j}^{\prime \prime}\right\}_{i, j=1, \ldots, p ;}^{h, k=1, \ldots, q ;}\right]
\end{aligned}
$$

with $X^{\prime}:=\left(x_{i, j}^{\prime}\right)_{i, j=1, \ldots, p ;}, X^{\prime \prime}:=\left(x_{h, k}^{\prime \prime}\right)_{h, k=1, \ldots, q}$; is strongly split as a Hopf superalgebra.

In any case, looking in detail we find that we have proved the following

Theorem 3.4.4. Every general linear $\mathbb{k}$-supergroup $\mathrm{GL}(V):=\mathrm{GL}_{p \mid q}$ is globally strongly split.

\section{Supergroups and Super Harish-Chandra Pairs}

Whether in a differential, analytic, or algebraic geometrical framework, with any given supergroup $G$ one can always associate, in a functorial way, its super HarishChandra pair (or sHCp for short), namely the pair $\left(G_{\mathbf{0}}, \mathfrak{g}\right)$ formed by the classical (even) subgroup $G_{\mathbf{0}}$ and the tangent Lie superalgebra $\mathfrak{g}:=\operatorname{Lie}(G)$ of $G$ itself. The key question is whether one can come back, and in the positive case what kind of (functorial) recipes one can explicitly provide to reconstruct the original supergroup out of its sHCp. In this section we present our own solutions to these problems, showing in particular that a positive answer is possible if and only if we restrict our attention to those (affine) supergroups which are globally strongly split — so fixing a link with the first half of the paper.

At first we shall deal with the linear case, i.e., with supergroups and sHCp's which are linearized. This is presented as a sheer source of inspiration, after which we treat the general case, which indeed might as well be dealt with independently.

\subsection{Super Harish-Chandra pairs.}

4.1.1. Super Harish-Chandra pairs. We now introduce the notion of a super HarishChandra pair, indeed a well-known one. Typically, it is considered in the framework of real or complex analytic super Lie groups (see [16] and [21] respectively). Here instead we consider the corresponding version adapted to the setup of algebraic supergroups in algebraic supergeometry (cf. [7], §3).

Definition 4.1.2. We call a super Harish-Chandra pair $(=s H C p)$ over $\mathbb{k}$ any pair $\left(G_{+}, \mathfrak{g}\right)$ such that

(a) $G_{+}$is an affine $\mathbb{k}$-group-scheme, $\mathfrak{g} \in(\mathrm{sLie})_{\mathbb{k}}$, and $\mathfrak{g}_{1}$ is a finite rank free $\mathbb{k}$-module;

(b) $\operatorname{Lie}\left(G_{+}\right)$is quasi-representable and $\operatorname{Lie}\left(G_{+}\right)=\mathfrak{g}_{0}$; 
(c) there is a $G_{+}-$action on $\mathfrak{g}$ by automorphisms, denoted $A d: G_{+} \longrightarrow \operatorname{Aut}(\mathfrak{g})$, such that its restriction to $\mathfrak{g}_{0}$ is the adjoint action of $G_{+}$on $\operatorname{Lie}\left(G_{+}\right)=\mathfrak{g}_{0}$ and the differential of this action is the Lie bracket of $\mathfrak{g}$ restricted to $\operatorname{Lie}\left(G_{+}\right) \times \mathfrak{g}=\mathfrak{g}_{0} \times \mathfrak{g}$.

All super Harish-Chandra pairs over $\mathbb{k}$ form the objects of a category, denoted $(\mathrm{sHCp})_{\mathbb{k}}$. The morphisms in $(\mathrm{sHCp})_{\mathbb{k}}$ are all pairs $\left(\Omega_{+}, \omega\right):\left(G_{+}^{\prime}, \mathfrak{g}^{\prime}\right) \longrightarrow$ $\left(G_{+}^{\prime \prime}, \mathfrak{g}^{\prime \prime}\right)$ of a morphism $\Omega_{+}: G_{+}^{\prime} \longrightarrow G_{+}^{\prime \prime}$ of $\mathbb{k}$-group schemes and a morphism $\omega: \mathfrak{g}^{\prime} \longrightarrow \mathfrak{g}^{\prime \prime}$ in $(\mathrm{sLie})_{\mathbb{k}}$ which are compatible with the additional sHCp structure, that is to say

$$
\text { (d) }\left.\omega\right|_{\mathfrak{g}_{0}}=d \Omega_{+}, \quad A d\left(\Omega_{+}(g)\right) \circ \omega=\omega \circ A d(g) \quad \forall g \in G_{+} .
$$

There is a natural, well-known way to attach an sHCp to any supergroup, which indeed motivates the very notion of sHCp. In the present context - letting (fsgroups) $)_{\mathbb{k}}$ be the category of fine $\mathbb{k}$-supergroups; see Definition 2.3.3 — it reads as follows:

Proposition 4.1.3. There exists a functor $\Phi:(\text { fsgroups })_{\mathbb{k}} \longrightarrow(\mathrm{sHCp})_{\mathbb{k}}$ given on objects by $\Phi: G \mapsto \Phi(G):=\left(G_{0}, \operatorname{Lie}(G)\right)$, and on morphisms by $\Phi: \varphi \mapsto$ $\Phi(\varphi):=\left(\left.\varphi\right|_{G_{0}}, \operatorname{Lie}(\varphi)\right)$.

4.1.4. The inversion problem for $\Phi$. The main question about the functor $\Phi$ : (fsgroups $)_{\mathbb{k}} \longrightarrow(\mathrm{sHCp})_{\mathbb{k}}$ is whether it is an equivalence. In down-to-earth terms, this amounts to asking: can one associate (backwards) a supergroup to any given sHCp, and can one reconstruct any supergroup from its associated sHCp (and conversely)? In order to answer this question, one looks for a quasi-inverse (i.e., "inverse up to isomorphism") functor to $\Phi$.

In the present, algebraic framework, a solution was given by Masuoka (see [19]) with the assumption that $\mathbb{k}$ be any field of characteristic different from 2 , using purely Hopf algebraic techniques. A weaker result is due to Carmeli and Fioresi (see [7]), who apply Koszul's original method (cf. [16]) to the context where the ground ring $\mathbb{k}$ be a field of characteristic zero; the same approach was recently extended to any commutative ring $\mathbb{k}$ by Masuoka and Shibata in [20].

In the next two subsections, we present yet another, totally general solution.

4.2. The converse functor: Linear case. In this subsection we present our own approach to solving the inversion problem explained in Section 4.1 .4 above, with a (functorial) geometrical method. The first approach that we follow is a representation-theoretical one: the basic ingredient to work with is an sHCp together with a faithful representation, which means that we restrict ourselves to linear sHCp's and linear supergroups. Later on, we adapt this construction to the general framework of all super Harish-Chandra pairs and all fine supergroups.

To start with, we define the notions of "linear" supergroups and super HarishChandra pairs.

\section{Definition 4.2.1.}

(a) We call linear gs-split fine supergroup over $\mathbb{k}$ any pair $(G, V)$ where $G \in$ (gss-fsgroups) $)_{\mathbb{k}}, V$ is a finite rank faithful $G$-module (that is, $V$ is a free $\mathbb{k}^{-}$ supermodule of finite rank such that $G$ embeds into $\mathrm{GL}(V)$ as a closed $\mathbb{k}$-supersubgroup), and $\mathfrak{g l}(V)_{\mathbf{1}} / \mathfrak{g}_{\mathbf{1}}$ is $\mathbb{k}$-free - or, what is the same, $\mathfrak{g l}(V)_{\mathbf{1}}=\mathfrak{g}_{\mathbf{1}} \oplus \mathfrak{q}$ for some $\mathbb{k}$-free submodule $\mathfrak{q}$ of $\mathfrak{g l}(V)_{1}$.

We denote by (lgss-fsgroups) $)_{\mathbb{k}}$ the category whose objects are linear supergroups over $\mathbb{k}$ and whose morphisms $\left(G^{\prime}, V^{\prime}\right) \longrightarrow\left(G^{\prime \prime}, V^{\prime \prime}\right)$ are given by pairs $\left(\varphi_{g}, \Phi_{v}\right)$ 
where $\varphi_{g}: G^{\prime} \longrightarrow G^{\prime \prime}$ and $\Phi_{v}: \mathrm{GL}\left(V^{\prime}\right) \longrightarrow \mathrm{GL}\left(V^{\prime \prime}\right)$ are morphisms of supergroups which obey an obvious compatibility constraint (namely, $\varphi_{g}$ is induced by $\Phi_{v}$ via restriction).

(b) We call linear super Harish-Chandra pair (over $\mathbb{k})$ any pair $\left(\left(G_{+}, \mathfrak{g}\right), V\right)$ where $\left(G_{+}, \mathfrak{g}\right) \in(\mathrm{sHCp})_{\mathbb{k}}$, the $V$ is a finite rank faithful $\left(G_{+}, \mathfrak{g}\right)$-module - this means, by definition, that $V$ is a free $\mathbb{k}$-supermodule of finite rank with representation monomorphisms $\boldsymbol{r}_{+}: G_{+} \longleftrightarrow \mathrm{GL}(V)$, as $\mathbb{k}$-supergroups (in particular, $G_{+}$is closed in $\left.\operatorname{GL}(V)\right)$, and $\rho: \mathfrak{g} \longleftrightarrow \mathfrak{g l}(V)$, as Lie $\mathbb{k}$-superalgebras, such that $\left.\rho\right|_{\mathfrak{g}_{\mathbf{0}}}=d \boldsymbol{r}_{+}, \quad \operatorname{Ad}\left(\boldsymbol{r}_{+}(g)\right) \circ \rho=\rho \circ \operatorname{Ad}(g)\left(\forall g \in G_{+}\right)$- and identifying $\mathfrak{g}_{\mathbf{1}}$ with $\rho\left(\mathfrak{g}_{1}\right)$, we have that $\mathfrak{g l}(V)_{\mathbf{1}} / \mathfrak{g}_{\mathbf{1}}$ is $\mathbb{k}$-free, or $\mathfrak{g l}(V)_{\mathbf{1}}=\mathfrak{g}_{\mathbf{1}} \oplus \mathfrak{q}$ for some finite $\mathbb{k}$-free submodule $\mathfrak{q}$.

We denote by $(\mathrm{lsHCp})_{\mathbb{k}}$ the category whose objects are linear super HarishChandra pairs over $\mathbb{k}$ and whose morphisms $\left(\left(G_{+}^{\prime}, \mathfrak{g}^{\prime}\right), V^{\prime}\right) \longrightarrow\left(\left(G_{+}^{\prime \prime}, \mathfrak{g}^{\prime \prime}\right), V^{\prime \prime}\right)$ are given by pairs $\left(\phi_{g}, \Phi_{v}\right)$ where $\phi_{g}:\left(G_{+}^{\prime}, \mathfrak{g}^{\prime}\right) \longrightarrow\left(G_{+}^{\prime \prime}, \mathfrak{g}^{\prime \prime}\right)$ is a morphism of super H-C pairs - i.e. in $(\mathrm{sHCp})_{\mathbb{k}}-\Phi_{v}: \mathrm{GL}\left(V^{\prime}\right) \longrightarrow \mathrm{GL}\left(V^{\prime \prime}\right)$ is a morphism of supergroups, and a natural (obvious) compatibility constraint linking $\phi_{g}$ and $\Phi_{v}$ holds.

Remark 4.2.2. It is worth recalling that the constraint for a supergroup to be linear is not that restrictive; indeed, it is well known that any (finite-dimensional) affine supergroup $G$ is linearizable - i.e., can be embedded inside some $\operatorname{GL}(V)$ - if its ground ring $\mathbb{k}$ is a field. Even more, the same is true - essentially by the same arguments - also when $\mathbb{k}$ is only a PID, under the additional assumption that $\mathcal{O}(G)$ be free as a $\mathbb{k}$-module.

It is easy to see from definitions that the functor $\Phi:(\text { fsgroups })_{\mathbb{k}} \longrightarrow(\mathrm{sHCp})_{\mathbb{k}}$ considered in Proposition 4.1 .3 above naturally induces a similar functor among the "associated linear" categories. The precise claim reads as follows:

Proposition 4.2.3. There is a unique functor $\Phi_{\ell}$ : (lgss-fsgroups $)_{\mathbb{k}} \longrightarrow\left(l_{\mathrm{sHCp}}\right)_{\mathbb{k}}$ which is given on objects by $\Phi_{\ell}:(G, V) \mapsto \Phi_{\ell}((G, V)):=\left(\left(G_{\mathbf{0}}, \operatorname{Lie}(G)\right), V\right)$.

We can now undertake the construction of a quasi-inverse functor to $\Phi_{\ell}$.

4.2.4. The functor $\Psi_{\ell}:(\mathrm{lsHCp})_{\mathbb{k}} \longrightarrow(\text { lgss-fsgroups })_{\mathbb{k}}$. Let us consider a linear sHCp over $\mathbb{k}$, say $\left(\left(G_{+}, \mathfrak{g}\right), V\right) \in(\mathrm{lsHCp})_{\mathbb{k}}$. As $G_{+}$embeds into $\mathrm{GL}(V)$, we identify $G_{+}$itself with its (closed) image inside $\mathrm{GL}(V)$; similarly, we identify $\mathfrak{g}$ with its image inside $\mathfrak{g l}(V)$. The very definition of a linear sHCp then tells us that the pair given by these two images do form a linear sHCp on its own.

We can now introduce the following definition:

Definition 4.2.5. Let $\mathcal{P}:=\left(\left(G_{+}, \mathfrak{g}\right), V\right) \in(\mathrm{lsHCp})_{\mathbb{k}}$. Let $1_{V} \in \mathfrak{g l}(V)$ be the identity endomorphism, fix in $\mathfrak{g}_{\mathbf{1}}$ (which is finite free) a $\mathbb{k}$-basis $\left\{Y_{i}\right\}_{i \in I}$ - for some finite index set $I$ - and fix also a total order in $I$. For all $A \in(\mathrm{salg})_{\mathbb{k}}$ consider in $\operatorname{GL}(V)(A)$ the set $\left(1_{V}+A_{\mathbf{1}} Y_{i}\right):=\left\{\left(1_{V}+\eta Y_{i}\right) \mid \eta \in A_{\mathbf{1}}\right\}$ for all $i \in I$. Then set

$$
G_{-}^{<}(A):=\overrightarrow{\prod_{i \in I}}\left(1_{V}+A_{\mathbf{1}} Y_{i}\right)=\left\{\overrightarrow{\prod_{i \in I}}\left(1_{V}+\eta_{i} Y_{i}\right) \mid \eta_{i} \in A_{\mathbf{1}} \forall i \in I\right\}
$$


where $\prod_{i \in I}$ denotes an ordered product (with respect to the fixed total order in $I$ ), and

$$
G_{\mathcal{P}}(A):=\left\langle G_{+}(A) \cup G_{-}^{<}(A)\right\rangle:=\left\langle G_{+}(A) \cup\left(\bigcup_{i \in I}\left(1_{V}+A_{1} Y_{i}\right)\right)\right\rangle
$$

the subgroup of $\mathrm{GL}(V)(A)$ generated by the subset $G_{+}(A) \cup G_{-}^{<}(A)$, or by $G_{+}(A)$ and the $\left(1_{V}+A_{1} Y_{i}\right)$ 's - with $G_{+}(A):=G_{+}\left(A_{\mathbf{0}}\right)$ by abuse of notation.

Finally, we denote by $G_{-}^{<}:(\mathrm{salg})_{\mathbb{k}} \longrightarrow$ (sets) and $G_{\mathcal{P}}:(\mathrm{salg})_{\mathbb{k}} \longrightarrow$ (groups) the $\mathbb{k}$-functor and the $\mathbb{k}$-supergroup functor defined by $A \mapsto G_{-}^{<}(A)$ and $A \mapsto$ $G_{\mathcal{P}}(A)$ - by the above recipes - on objects and in the obvious way on morphisms.

N.B.: by definition $G_{-}^{<}$depends on the choice of the $\mathbb{k}$-basis $\left\{Y_{i}\right\}_{i \in I}$ of $\mathfrak{g}_{\mathbf{1}}$. On the other hand, we shall presently see that $G_{\mathcal{P}}$ instead is independent of such a choice.

Lemma 4.2.6. Let $G \in$ (sgroups) $)_{\mathbb{k}}$ be a $\mathbb{k}$-supergroup such that Lie $(G)$ is quasirepresentable, say Lie $(G)=\mathcal{L}_{\mathfrak{g}}$ for some $\mathfrak{g} \in(\mathrm{sLie})_{\mathbb{k}}$. Let $A \in(\mathrm{salg})_{\mathbb{k}}, \eta, \eta^{\prime}, \eta^{\prime \prime} \in$ $A_{\mathbf{1}}, c \in A_{\mathbf{0}}$ such that $c^{2}=0, Y, Y^{\prime} \in \mathfrak{g}_{\mathbf{1}}, X \in \mathfrak{g}_{\mathbf{0}}$ and $g_{0} \in G_{0}(A)$. Then we have (notation of Definition 2.2.1)

(a) $(1+c X) \in G_{\mathbf{0}}(A),(1+\eta Y) \in G(A) ; \quad$ in particular $\left(1+\eta \eta^{\prime}\left[Y, Y^{\prime}\right]\right) \in$ $G_{\mathbf{0}}(A)$;

(b) $(1+\eta Y) g_{0}=g_{0}\left(1+\eta A d\left(g_{0}^{-1}\right)(Y)\right)$;

(c) $\left(1+\eta^{\prime} Y^{\prime}\right)\left(1+\eta^{\prime \prime} Y^{\prime \prime}\right)=\left(1+\eta^{\prime \prime} \eta^{\prime}\left[Y^{\prime}, Y^{\prime \prime}\right]\right)\left(1+\eta^{\prime \prime} Y^{\prime \prime}\right)\left(1+\eta^{\prime} Y^{\prime}\right)$;

(d) $\left(1+\eta Y^{\prime}\right)\left(1+\eta Y^{\prime \prime}\right)=\left(1+\eta\left(Y^{\prime}+Y^{\prime \prime}\right)\right)=\left(1+\eta Y^{\prime \prime}\right)\left(1+\eta Y^{\prime}\right)$;

(e) $\left(1+\eta^{\prime} Y\right)\left(1+\eta^{\prime \prime} Y\right)=\left(1+\eta^{\prime \prime} \eta^{\prime} Y^{\langle 2\rangle}\right)\left(1+\left(\eta^{\prime}+\eta^{\prime \prime}\right) Y\right)$;

(f) $(1+\eta Y)\left(1+\eta^{\prime} \eta^{\prime \prime} X\right)=\left(1+\eta^{\prime} \eta^{\prime \prime} X\right)\left(1+\eta \eta^{\prime} \eta^{\prime \prime}[Y, X]\right)(1+\eta Y)$ $=\left(1+\eta^{\prime} \eta^{\prime \prime} X\right)(1+\eta Y)\left(1+\eta \eta^{\prime} \eta^{\prime \prime}[Y, X]\right)$.

(g) Let $(h, k):=h k h^{-1} k^{-1}$ be the commutator of elements $h$ and $k$ in a group. Then

$\left((1+\eta Y),\left(1+\eta^{\prime} Y^{\prime}\right)\right)=\left(1+\eta^{\prime} \eta\left[Y, Y^{\prime}\right]\right), \quad\left((1+\eta Y),\left(1+\eta Y^{\prime}\right)\right)=\left(1+\eta\left(Y+Y^{\prime}\right)\right)$, $\left(\left(1+\eta^{\prime} Y\right),\left(1+\eta^{\prime \prime} Y\right)\right)=\left(1+\eta^{\prime \prime} \eta^{\prime} Y^{\langle 2\rangle}\right)^{2}=\left(1+\eta^{\prime \prime} \eta^{\prime} 2 Y^{\langle 2\rangle}\right)=\left(1+\eta^{\prime \prime} \eta^{\prime}[Y, Y]\right)$.

(N.B.: taking the rightmost term in the last identity, the latter is a special case of the first.)

Proof. Recall that $G(A)=\operatorname{Hom}_{(\operatorname{salg})_{\mathrm{k}}}(\mathcal{O}(G), A) \subseteq \operatorname{Hom}_{(\operatorname{smod})_{\mathrm{k}}}(\mathcal{O}(G), A)$, the latter being the $\mathbb{k}$-supermodule of all morphisms between $\mathcal{O}(G)$ and $A$ in the category of $\mathbb{k}$-supermodules; the sum in the formulas then is just the sum in the $\mathbb{k}$-supermodule $\operatorname{Hom}_{(\operatorname{smod})_{\mathfrak{k}}}(\mathcal{O}(G), A)$. Also, in those formulas the "1" stands for the unit element in $G(A)=\operatorname{Hom}_{(\mathrm{salg})_{\mathrm{k}}}(\mathcal{O}(G), A)$, which is the map given by composition $1:=1_{G(A)}: \mathcal{O}(G) \stackrel{\epsilon_{\mathcal{O}(G)}}{\longrightarrow} \mathbb{k} \stackrel{u_{A}}{\longrightarrow} A$.

Once this is fixed, everything follows easily by straightforward calculations and from the identities $\left(1+\eta \eta^{\prime}\left[Y, Y^{\prime}\right]\right)=\exp \left(\eta \eta^{\prime}\left[Y, Y^{\prime}\right]\right)$ (see [6], $\S 11.5$, for details), $Y^{2}=Y^{\langle 2\rangle}$ and $g_{0}^{-1} Y g_{0}=A d\left(g_{0}^{-1}\right)(Y)$, which do hold in any representation of $\mathfrak{g}$. In particular, claim $(g)$ directly follows from the identities in $(c),(d)$ and $(e)$.

It is possibly worth adding some details for claim (a), which holds by extending to the present super-context a standard trick for group-schemes.

Let $(a, Z)$ be $(c, X)$ or $(\eta, Y)$. From $Z \in \mathfrak{g}$ we have

$$
a Z:=a \otimes Z \in\left(A_{\mathbb{k}} \otimes \mathfrak{g}\right)_{\mathbf{0}}=: \mathfrak{g}(A) .
$$


By the standard identification of $\mathfrak{g}(A)=\mathcal{L}_{\mathfrak{g}}(A)$ with $\operatorname{Lie}(G)(A):=\operatorname{Ker}\left(G(p)_{A}\right)$ - see Section 2.3 and the references therein - we have that $(1+\varepsilon a Z) \in G(A[\varepsilon])$. But now, as $\varepsilon^{2}=0$, the fact that $(1+\varepsilon a Z) \in G(A[\varepsilon])=\operatorname{Hom}_{\left(\operatorname{salg}_{\mathrm{k}}\right.}(\mathcal{O}(G), A[\varepsilon])$ be multiplication preserving is equivalent to the fact that $a Z \in \operatorname{Hom}_{(\operatorname{smod})_{\mathrm{k}}}(\mathcal{O}(G), A)$ be an $A$-valued $\epsilon_{\mathcal{O}(G)}$-derivation, i.e.,

$$
a Z\left(f^{\prime} \cdot f^{\prime \prime}\right)=a Z\left(f^{\prime}\right) \cdot \epsilon_{\mathcal{O}(G)}\left(f^{\prime \prime}\right)+\epsilon_{\mathcal{O}(G)}\left(f^{\prime}\right) \cdot a Z\left(f^{\prime \prime}\right) \quad \forall f^{\prime}, f^{\prime \prime} \in \mathcal{O}(G) .
$$

But then, in turn, as $a \in\{c, \eta\}$ also satisfies $a^{2}=0$, we have that $(1+a Z)$ is multiplication preserving too, so that $(1+a Z) \in \operatorname{Hom}_{(\mathrm{salg})_{\mathrm{k}}}(\mathcal{O}(G), A)=$ : $G(A)$.

This lemma is the key to proving the next relevant result.

Proposition 4.2.7. For any $A \in(\mathrm{salg})_{\mathbb{k}}$, there exist group-theoretic factorizations

$$
G_{\mathcal{P}}(A)=G_{+}(A) \cdot G_{-}^{<}(A), \quad G_{\mathcal{P}}(A)=G_{-}^{<}(A) \cdot G_{+}(A) .
$$

Moreover, the group $G_{\mathcal{P}}(A)$ is independent of the choice of an ordered $\mathbb{k}$-basis $\left\{Y_{i}\right\}_{i \in I}$ of $\mathfrak{g}_{1}$ used for its definition; the same holds true for the whole functor $G_{\mathcal{P}}$. Similarly, the sets $G_{+}\left(A_{1}^{(2)}\right) G_{-}^{<}(A)$ and $G_{-}^{<}(A) G_{+}\left(A_{1}^{(2)}\right)-c f$. Section 2.1.1 both coincide with the subgroup of $G_{\mathcal{P}}(A)$ generated by $G_{+}\left(A_{1}^{(2)}\right)$ and $G_{-}^{<}(A)$, and they are independent of the choice of an ordered $\mathbb{k}$-basis of $\mathfrak{g}_{\mathbf{1}}$.

Proof. First of all, as we need it later on, we notice that the even part of $G_{\mathcal{P}}$ is, directly from definitions, nothing but $G_{+}$, i.e., $\left(G_{\mathcal{P}}\right)_{0}=G_{+}$. Definitions imply also that the inverse of any element $\left(1+\eta_{i} Y_{i}\right) \in G_{-}^{<}(A)$ is nothing but $\left(1+\eta_{i} Y_{i}\right)^{-1}=$ $\left(1-\eta_{i} Y_{i}\right) \in G_{-}^{<}(A)$. Taking this into account, our goal amounts to showing that

$$
g_{+}^{\prime} \prod_{i \in I}^{\rightarrow}\left(1+\eta_{i}^{\prime} Y_{i}\right) \cdot g_{+}^{\prime \prime} \prod_{i \in I}^{\rightarrow}\left(1+\eta_{i}^{\prime \prime} Y_{i}\right) \in G_{+}(A) \cdot G_{-}^{<}(A)
$$

for all $g_{+}^{\prime}, g_{+}^{\prime \prime} \in G_{+}(A)$ and $\eta_{i}^{\prime}, \eta_{i}^{\prime \prime} \in A_{\mathbf{1}}$, i.e., we can re-write $g_{+}^{\prime} \prod_{i \in I}^{\rightarrow}\left(1+\eta_{i}^{\prime} Y_{i}\right)$. $g_{+}^{\prime \prime} \prod_{i \in I}\left(1+\eta_{i}^{\prime \prime} Y_{i}\right)$ as the product of an element in $G_{+}(A)$ times an ordered product of factors of type $\left(1+\eta_{\ell} Y_{\ell}\right)$.

First of all, claim (b) of Lemma 4.2.6 gives (for all $i \in I$ )

$$
\left(1+\eta_{i}^{\prime} Y_{i}\right) g_{+}^{\prime \prime}=g_{+}^{\prime \prime}\left(1+\eta_{i}^{\prime} A d\left(\left(g_{+}^{\prime \prime}\right)^{-1}\right)\left(Y_{i}\right)\right)=g_{+}^{\prime \prime}\left(1+\eta_{i}^{\prime} \sum_{j \in I} c_{i, j} Y_{j}\right)
$$

for some $c_{i, j} \in \mathbb{k}(j \in I)$. But now the special case of claim (d) in Lemma 4.2.6 implies

$$
\left(1+\eta_{i}^{\prime} \sum_{j \in I} c_{i, j} Y_{j}\right)=\prod_{j \in I}\left(1+\eta_{i}^{\prime} c_{i, j} Y_{j}\right)=\prod_{j \in I}^{\rightarrow}\left(1+\eta_{i}^{\prime} c_{i, j} Y_{j}\right) \in G_{-}^{<}(A)
$$

as in particular the factors in the product(s) do commute among themselves.

Applying all this to $g_{+}^{\prime} \vec{\prod}_{i \in I}\left(1+\eta_{i}^{\prime} Y_{i}\right) \cdot g_{+}^{\prime \prime} \vec{\prod}_{i \in I}\left(1+\eta_{i}^{\prime \prime} Y_{i}\right)$ we eventually find $g_{+}^{\prime} \overrightarrow{\prod_{i \in I}}\left(1+\eta_{i}^{\prime} Y_{i}\right) \cdot g_{+}^{\prime \prime} \prod_{i \in I}^{\vec{D}}\left(1+\eta_{i}^{\prime \prime} Y_{i}\right)=\left(g_{+}^{\prime} g_{+}^{\prime \prime}\right) \cdot \overrightarrow{\prod_{i \in I}} \prod_{j \in I}^{\overrightarrow{1}}\left(1+\eta_{i}^{\prime} c_{i, j} Y_{j}\right) \overrightarrow{\prod_{i \in I}}\left(1+\eta_{i}^{\prime \prime} Y_{i}\right)$ in which the first factor $\left(g_{+}^{\prime} g_{+}^{\prime \prime}\right)$ of the right hand side does belong to $G_{+}(A)$. Therefore, in order to prove (4.1) we are left to show that the following holds. 
Claim. Any (possibly unordered) product of the form $\prod_{k=1}^{N}\left(1+\eta_{k} Y_{i_{k}}\right)$ can be "reordered", i.e., it can be re-written as an element of $G_{+}\left(A_{1}^{(2)}\right) \cdot G_{-}^{<}(A)$.

In order to prove the claim, let $\mathfrak{a}$ be the (two-sided) ideal of $A$ generated by the $\eta_{k}$ 's, and denote by $\mathfrak{a}^{n}$ its $n$-th power, for any $n \in \mathbb{N}$. As the $\eta_{k}$ 's are finitely many odd elements, we have $\mathfrak{a}^{n}=\{0\}$, for all $n>N$.

Looking at the product $\prod_{k=1}^{N}\left(1+\eta_{k} Y_{i_{k}}\right)$, we define its inversion number as being the number of occurrences of two consecutive indices $k_{s}$ and $k_{s+1}$ such that $i_{k_{s}} \npreceq i_{k_{s+1}}$. The product itself then is ordered iff its inversion number is zero.

Now assume the product $g:=\prod_{k=1}^{N}\left(1+\eta_{k} Y_{i_{k}}\right)$ is unordered; then there exists at least an inversion, say $i_{k_{s}} \npreceq i_{k_{s+1}}$, i.e., either $i_{k_{s}} \succ i_{k_{s+1}}$ or $i_{k_{s}}=i_{k_{s+1}}$. Using claim (c) or (e), respectively, of Lemma 4.2.6 we can re-write the product $\left(1+\eta_{k_{s}} Y_{i_{k_{s}}}\right)\left(1+\eta_{k_{s+1}} Y_{i_{k_{s+1}}}\right)$ as

$$
\begin{gathered}
\left(1+\eta_{k_{s}} Y_{i_{k_{s}}}\right)\left(1+\eta_{k_{s+1}} Y_{i_{k_{s}+1}}\right) \\
=\left(1+\eta_{k_{s+1}} \eta_{k_{s}}\left[Y_{i_{k_{s}+1}}, Y_{i_{k_{s}}}\right]\right)\left(1+\eta_{k_{s+1}} Y_{i_{k_{s}+1}}\right)\left(1+\eta_{k_{s}} Y_{i_{k_{s}}}\right) \\
\left(1+\eta_{k_{s}} Y_{i_{k_{s}}}\right)\left(1+\eta_{k_{s+1}} Y_{i_{k_{s}}}\right) \\
=\left(1+\eta_{k_{s+1}} \eta_{k_{s}} Y_{i_{k_{s}}}^{\langle 2\rangle}\right)\left(1+\left(\eta_{k_{s}}+\eta_{k_{s+1}}\right) Y_{i_{k_{s}}}\right) .
\end{gathered}
$$

Thus, re-writing in this way the product of the $k_{s}$-th and the $k_{s+1}$-th factor in the original product $g:=\prod_{k=1}^{N}\left(1+\eta_{k} Y_{i_{k}}\right)$, we find another product expression in which we did eliminate one inversion, but we paid the price of inserting a new factor. However, in both cases this new factor is of the form $(1+a X)$ for some $X \in \mathfrak{g}_{0} \in G_{+}(A)$ and $a \in \mathfrak{a}^{2}$.

By repeated use of Lemma 4.2.6 $(f)$ we can shift this new factor $(1+a X)$ to the leftmost position in $g$ (now re-written once more in yet a different product form) up to paying the price of inserting several new factors of the form $\left(1+b_{t} Z_{t}\right)$ for some $Z_{t} \in \mathfrak{g}_{1}$ and $b_{t} \in \mathfrak{a}^{3}$. Moreover, by Lemma 4.2.6 (d) each one of these new factors can be written as a product of factors of the form $\left(1+\eta_{h}^{\prime} Y_{i_{h}^{\prime}}\right)$ where $\eta_{h}^{\prime} \in A_{\mathbf{1}}$ is a multiple of some $b_{t}$, hence $\eta_{h}^{\prime} \in \mathfrak{a}^{3}$ too.

Eventually, we find a new factorization of the original element

$$
g:=\prod_{k=1}^{N}\left(1+\eta_{k} Y_{i_{k}}\right)
$$

in the new form

$$
g:=g_{0}^{\prime} \cdot \prod_{h=1}^{N^{\prime}}\left(1+\eta_{h}^{\prime} Y_{i_{h}^{\prime}}\right)
$$

where $g_{0}^{\prime} \in G_{+}(A)$ and the factors $\left(1+\eta_{h}^{\prime} Y_{i_{h}^{\prime}}\right)$ satisfy the following conditions:

- (a) each new factor $\left(1+\eta_{h}^{\prime} Y_{i_{h}^{\prime}}\right)$ is either one of the old factors $\left(1+\eta_{k} Y_{i_{k}}\right)$ or a truly new one;

- (b) for every (truly) new factor $\left(1+\eta_{h}^{\prime} Y_{i_{h}^{\prime}}\right)$ one has $\eta_{h}^{\prime} \in \mathfrak{a}^{3}$;

- (c) the number of inversions among factors $\left(1+\eta_{h}^{\prime} Y_{i_{h}^{\prime}}\right)=\left(1+\eta_{k} Y_{i_{k}}\right)$ of the old type is one less than before. 
Iterating this procedure, after finitely many steps we can achieve a new factorization of the original element $g:=\prod_{k=1}^{N}\left(1+\eta_{k} Y_{i_{k}}\right)$ as a new product $g=$ $g_{0}^{\prime \prime} \cdot \prod_{h=1}^{N^{\prime \prime}}\left(1+\eta_{h}^{\prime \prime} Y_{i_{h}^{\prime \prime}}\right)$ where $g_{0}^{\prime \prime} \in G_{+}(A)$ and the factors $\left(1+\eta_{h}^{\prime \prime} Y_{i_{h}^{\prime \prime}}\right)$ enjoy properties (a) and (b) above plus the "optimal version" of (c), namely

- $(c+)$ the number of inversions among factors of the old type is zero.

Now we apply the same "reordering operation" to the product $\prod_{h=1}^{N^{\prime \prime}}\left(1+\eta_{h}^{\prime \prime} Y_{i_{h}^{\prime \prime}}\right)$. By assumption, now an inversion can occur only among two factors of new type or among an old and a new factor. But then, the two coefficients $\eta_{h}^{\prime \prime}$ involved by the inversion belong to $\mathfrak{a}$ and at least one of them belong to $\mathfrak{a}^{3}$. It follows that when one performs the "reordering operation" onto the pair of factors involved in the inversion the new factor which pops up necessarily involves a coefficient in $\mathfrak{a}^{4}$. As this applies for any possible inversion, at the end of the day we shall find a new factorization of $g$ of the form

$$
g=g_{0}^{\prime \prime} \cdot \widehat{g}_{0} \cdot \prod_{t=1}^{\widehat{N}}\left(1+\widehat{\eta}_{t} Y_{\widehat{i}_{t}}\right)
$$

in which $\widehat{g}_{0} \in G_{+}(A)$ and the factors $\left(1+\widehat{\eta}_{t} Y_{\widehat{i}_{t}}\right)$ are either old factors $\left(1+\eta_{k} Y_{i_{k}}\right)$, with no inversions among them, or new factors for which $\widehat{\eta}_{t} \in \mathfrak{a}^{5}$.

The end of the story is clear. We can iterate at will this procedure, and then since $\mathfrak{a}^{n}=\{0\}$ for $n>N$ - after finitely many steps we have no longer any new factor popping out; thus, we eventually find a last factorization of $g$ of the form

$$
g=\widetilde{g}_{0} \cdot \prod_{\ell=1}^{\widetilde{N}}\left(1+\widetilde{\eta}_{\ell} Y_{\widetilde{i}_{\ell}}\right)=\widetilde{g}_{0} \cdot \prod_{\prod_{\ell=1}^{N}}^{\widetilde{N}}\left(1+\widetilde{\eta}_{\ell} Y_{\widetilde{i}_{\ell}}\right)
$$

in which $\widetilde{g}_{0} \in G_{+}(A)$ and $\prod_{\ell=1}^{\widetilde{N}}\left(1+\widetilde{\eta}_{\ell} Y_{\widetilde{i}_{\ell}}\right)=\prod_{\prod_{\ell=1}^{\widetilde{N}}}^{\widetilde{N}}\left(1+\widetilde{\eta}_{\ell} Y_{\widetilde{i}_{\ell}}\right) \in G_{-}^{<}(A)$ is an ordered product, as required, so that $g \in G_{+}(A) \cdot G_{-}^{<}(A)$.

For the last part of the main statement, let $\left\{Y_{i}\right\}_{i \in I}$ and $\left\{Z_{i}\right\}_{i \in I}$ be two (finite) $\mathbb{k}$-bases of $\mathfrak{g}_{1}$; then $Z_{j}=\sum_{i \in I} c_{i} Y_{i}$ (with $c_{i} \in I$ ) for each $j \in I$. The same argument proving (4.2) also yields

$$
1+\psi_{j} Z_{j}=1+\psi_{j} \sum_{i \in I} c_{i} Y_{i}=\overrightarrow{\prod_{i \in I}}\left(1+\psi_{j} c_{i} Y_{i}\right) \in G_{-}^{<, Y}(A)
$$

where $G_{-}^{<, Y}$ is relative to the group $G_{p}^{Y}$ defined as in Definition 4.2.5 making use of the basis of the $\left\{Y_{i}\right\}_{i \in I}$. Letting $G_{-}^{<, Z}$ be the similar group defined via the basis of the $Z_{j}$ 's, formula (4.3) proves that $G_{\mathcal{P}}^{<, Z}(A) \subseteq G_{+}\left(A_{1}^{(2)}\right) G_{\mathcal{P}}^{<, Y}(A)$, so by symmetry we eventually get $G_{+}\left(A_{1}^{(2)}\right) G_{\mathcal{P}}^{<, Z}(A)=G_{+}\left(A_{1}^{(2)}\right) G_{\mathcal{P}}^{<, Y}(A)$.

To improve the previous result, we need a couple of additional lemmas.

Lemma 4.2.8. Let $A \in(\mathrm{salg})_{\mathbb{k}}$, let $\hat{\eta}_{i}, \check{\eta}_{i} \in A_{\mathbf{1}}$ and let $\mathfrak{q}$ be an ideal of $A$ such that $\hat{\eta}_{i}, \check{\eta}_{i} \in \mathfrak{q}$ and $\alpha_{i}:=\hat{\eta}_{i}-\check{\eta}_{i} \in \mathfrak{q}^{n}(i \in I)$ for some $n \in \mathbb{N}$. Then

$$
\overrightarrow{\prod_{i \in I}}\left(1+\left[\hat{\eta}_{i}\right]_{n+1} Y_{i}\right) \cdot \overleftarrow{\prod_{i \in I}}\left(1-\left[\check{\eta}_{i}\right]_{n+1} Y_{i}\right)=\overrightarrow{\prod_{i \in I}}\left(1+\left[\alpha_{i}\right]_{n+1} Y_{i}\right) \in G_{\mathcal{P}}\left(A / \mathfrak{q}^{n+1}\right)
$$

where $\vec{\prod}_{i \in I}$ and $\overleftarrow{\prod}_{i \in I}$ respectively denote an ordered and a reversely-ordered product (w.r.t. the given order in $I$ ) and $[a]_{n+1} \in A / \mathfrak{q}^{n+1}$ stands for the coset modulo $\mathfrak{q}^{n+1}$ of any $a \in A$. 
Proof. This is an easy, straightforward consequence of claims (e) and $(f)$ in Lemma 4.2 .6 .

Lemma 4.2.9. For any given $A \in(\mathrm{salg})_{\mathbb{k}}$, let $\zeta_{i} \in A_{\mathbf{1}}(i \in I)$ be such that

$$
g:=\prod_{i \in I}\left(1+\zeta_{i} Y_{i}\right) \in G_{+}(A) \cap G_{-}^{<}(A) .
$$

Then $\zeta_{i}=0$ for all $i \in I$.

Proof. By our global assumptions we have $G_{\mathcal{P}}(A) \subseteq \mathrm{GL}(V)(A) \subseteq \operatorname{End}_{\mathbb{k}}(V)(A)$, with $\operatorname{End}_{\mathbb{k}}(V)(A)$ being a unital, associative $A_{\mathbf{0}^{-}}$-algebra. Indeed, fixing a homogeneous $\mathbb{k}$-bases for $V$ we can read $\operatorname{End}_{\mathbb{k}}(V)(A)$ as an algebra of block matrices, in which the diagonal blocks have entries in $A_{\mathbf{0}}$ and the other ones have entries in $A_{\mathbf{1}}$ (like in Example 2.1.5(b) and the references therein). Thus, inside $\operatorname{End}_{\mathbb{k}}(V)(A)$ we can expand the product $g:=\vec{\prod}_{i \in I}\left(1+\zeta_{i} Y_{i}\right) \in G_{\mathcal{P}}(A) \subseteq \operatorname{End}_{\mathbb{k}}(V)(A)$ so to get

$$
g:=\overrightarrow{\prod_{i \in I}}\left(1+\zeta_{i} Y_{i}\right)=1+\sum_{n \in \mathbb{N}_{+}} c_{n}(\underline{\zeta})
$$

where each $c_{n}(\underline{\zeta})$ denotes a (block) matrix in $\operatorname{End}_{\mathbb{k}}(V)(A)$ whose entries are homogeneous polynomials in the $\zeta_{i}$ 's of degree $n$.

In particular we have $c_{n}(\underline{\zeta})=0$ for all $n>|I|$, and moreover $c_{1}(\underline{\zeta})=$ $\sum_{i \in I} \zeta_{i} Y_{i}$

Now let $\mathfrak{a}:=\left(\left\{\zeta_{i}\right\}_{i \in I}\right)$ be the ideal of $A$ generated by all the $\zeta_{i}$ 's. For all $n \in \mathbb{N}$, let $p_{n}: A \longrightarrow A / \mathfrak{a}^{n}=:[A]_{n}$ be the canonical quotient map, for which we write $[a]_{n}:=p_{n}(a)$ for every $a \in A$. Correspondingly, we let $G_{\mathcal{P}}\left(p_{n}\right)$ : $G_{\mathcal{P}}(A) \longrightarrow G_{\mathcal{P}}\left(A / \mathfrak{a}^{n}\right)=: G_{\mathcal{P}}\left([A]_{n}\right)$ be the associated group morphism and we write $[y]_{n}:=G_{\mathcal{P}}\left(p_{n}\right)(y)$ for every $y \in G_{\mathcal{P}}(A)$.

Applying this to (4.4) above we find

$$
[g]_{2}:=\left[1+c_{1}(\underline{\zeta})\right]_{2}=1+\sum_{i \in I}\left[\zeta_{i}\right]_{2} Y_{i} \in G_{\mathcal{P}}\left([A]_{2}\right) .
$$

On the other hand, the assumption $g \in G_{+}(A) \cap G_{-}^{<}(A)$ implies also $[g]_{2} \in$ $G_{+}\left([A]_{2}\right)$, which means that all entries — belonging to $[A]_{2}$ - of the matrix $[g]_{2}$ actually belong to the even part of $[A]_{2}$. This together with (4.5) forces $\sum_{i \in I}\left[\zeta_{i}\right]_{2} Y_{i}=0$. In turn, by the linear independence of the $Y_{i}$ 's - inside $\mathfrak{g}_{1}$, hence inside $A_{\mathbf{1}} \cdot \mathfrak{g}_{\mathbf{1}} \subseteq\left(A \otimes_{\mathbb{k}} \mathfrak{g}\right)_{\mathbf{0}} \subseteq \operatorname{End}(V)(A)-$ this implies $\left[\zeta_{i}\right]_{2}=[0]_{2} \in$ $[A]_{2}:=A / \mathfrak{a}^{2}$, hence $\zeta_{i} \in \mathfrak{a}^{2}$ for all $i \in I$.

Now, $\left\{\zeta_{i}\right\}_{i \in I} \subseteq \mathfrak{a}^{2}=\left(\left\{\zeta_{i}\right\}_{i \in I}\right)^{2}$ automatically entails $\left\{\zeta_{i}\right\}_{i \in I} \subseteq \mathfrak{a}^{n}$ for all $n \in \mathbb{N}_{+}$. As $\mathfrak{a}^{n}=\{0\}$ for $n \gg 0$, we end up with $\zeta_{i}=0$ for all $i \in I$.

Remark 4.2.10. An alternative argument to finish the previous proof is the following. Once we have found that $\zeta_{i} \in \mathfrak{a}^{2}$ for all $i \in I$, we remark that this implies $c_{n}(\underline{\zeta}) \in \mathfrak{a}^{2 n}$ for all $n \in \mathbb{N}_{+}$. Then (4.4) yields the analogue of (4.5), namely

$$
[g]_{4}:=\left[1+c_{1}(\underline{\zeta})\right]_{4}=1+\sum_{i \in I}\left[\zeta_{i}\right]_{4} Y_{i} \in G_{\mathcal{P}}\left([A]_{4}\right)
$$

and again, acting like above, by a parity argument in $G_{\mathcal{P}}\left([A]_{4}\right)$ along with the linear independence of the $Y_{i}$ 's we get $\zeta_{i} \in \mathfrak{a}^{4}$ for all $i \in I$. 
We can now iterate this procedure, thus finding $\zeta_{i} \in \mathfrak{a}^{2 n}$ (for $i \in I$ ) for all $n \in \mathbb{N}_{+}$. As $\mathfrak{a}^{2 n}=\{0\}$ for $n \gg 0$, we end up with $\zeta_{i}=0$ for all $i \in I$.

Thanks to the previous lemmas, we can improve Proposition 4.2.7 as follows:

\section{Proposition 4.2.11.}

(a) The restriction of group multiplication in $G_{\mathcal{P}}$ provides $\mathbb{k}$-superscheme isomorphisms

$$
G_{+} \times G_{-}^{<} \cong G_{\mathcal{P}}, \quad G_{-}^{<} \times G_{+} \cong G_{\mathcal{P}} .
$$

(b) There exist $\mathbb{k}$-superscheme isomorphisms $G_{-}^{<} \cong \chi_{i \in I} \mathbb{A}_{\mathbb{k}}^{0 \mid 1} \cong \mathbb{A}_{\mathbb{k}}^{0 \mid d-}$ with $d_{-}:=|I|$.

Proof. (a) It is enough to prove the first identity, which amounts to showing the following: for any $A \in(\mathrm{salg})_{\mathbb{k}}$, if $\hat{g}_{+} \hat{g}_{-}=\check{g}_{+} \check{g}_{-}$for $\hat{g}_{ \pm}, \check{g}_{ \pm} \in G_{ \pm}(A)$, then $\hat{g}_{+}=\check{g}_{+}$and $\hat{g}_{-}=\check{g}_{-}$.

From the assumption $\hat{g}_{+} \hat{g}_{-}=\check{g}_{+} \check{g}_{-}$we get $g:=\hat{g}_{-} \check{g}_{-}^{-1}=\hat{g}_{+}^{-1} \check{g}_{+} \in$ $G_{+}(A)$, as $G_{+}(A)$ is a subgroup in $G(A)$. Now $\hat{g}_{-} \in G_{-}^{<}(A)$ has the form $\hat{g}_{-}=$ $\vec{\prod}_{i \in I}\left(1+\hat{\eta}_{i} Y_{i}\right)$ and similarly $\check{g}_{-}=\vec{\prod}_{i \in I}\left(1+\check{\eta}_{i} Y_{i}\right)$ so that $\check{g}_{-}^{-1}=\overleftarrow{\prod}_{i \in I}\left(1-\check{\eta}_{i} Y_{i}\right)$ where once more $\vec{\prod}$ and $\overleftarrow{\prod}$ respectively denote an ordered and a reversely-ordered product. Therefore we have

$$
g:=\hat{g}_{-} \check{g}_{-}^{-1}=\prod_{i \in I}^{\rightarrow}\left(1+\hat{\eta}_{i} Y_{i}\right) \overleftarrow{\prod_{i \in I}}\left(1-\check{\eta}_{i} Y_{i}\right) \in G_{+}(A) \subseteq G_{\mathcal{P}}(A)
$$

We define $\mathfrak{a}:=\left(\left\{\hat{\eta}_{i}, \check{\eta}_{i}\right\}_{i \in I}\right)$ the ideal of $A$ generated by all the $\hat{\eta}_{i}$ 's and the $\check{\eta}_{i}$ 's. Like in the proof of Lemma 4.2.9, for $n \in \mathbb{N}$ we write $p_{n}: A \longrightarrow A / \mathfrak{a}^{n}=$ : $[A]_{n}$ for the canonical quotient map and $[a]_{n}:=p_{n}(a)$ for every $a \in A$, and then also, correspondingly, $G_{\mathcal{P}}\left(p_{n}\right): G_{\mathcal{P}}(A) \longrightarrow G_{\mathcal{P}}\left(A / \mathfrak{a}^{n}\right)=: G_{\mathcal{P}}\left([A]_{n}\right)$ for the associated group morphism and $[y]_{n}:=G_{\mathcal{P}}\left(p_{n}\right)(y)$ for every $y \in G_{\mathcal{P}}(A)$. Now (4.6) along with Lemma 4.2 .8 gives

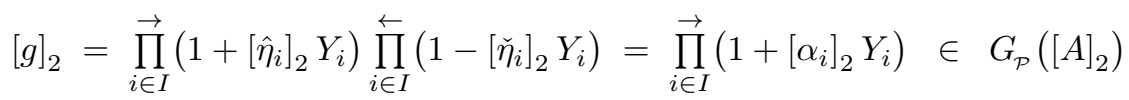

with $\alpha_{i}:=\hat{\eta}_{i}-\check{\eta}_{i} \in \mathfrak{a}$ for all $i$. Since it is also $[g]_{2} \in G_{+}\left([A]_{2}\right) \cap G_{-}^{<}\left([A]_{2}\right)$, we can apply Lemma 4.2.9, with $[A]_{2}$ playing the rôle of $A$, giving $\left[\alpha_{i}\right]_{2}=[0]_{2} \in[A]_{2}$, that is, $\alpha_{i} \in \mathfrak{a}^{2}$, for all $i \in I$. But now Lemma 4.2.9 applies again, with $[A]_{3}$ playing the rôle of $A$, yielding $\left[\alpha_{i}\right]_{3}=[0]_{3} \in[A]_{3}$ hence $\alpha_{i} \in \mathfrak{a}^{3}$, for all $i \in I$. Then we iterate, finding by induction that $\alpha_{i} \in \mathfrak{a}^{n}$ (for $i \in I$ ) for all $n \in \mathbb{N}_{+}$; as $\mathfrak{a}^{n}=\{0\}$ for $n \gg 0$ we end up with $\hat{\eta}_{i}-\check{\eta}_{i}=: \alpha_{i}=0$, i.e., $\hat{\eta}_{i}=\check{\eta}_{i}$, for all $i \in I$. This yields $\hat{g}_{-}=\check{g}_{-}$, and from this we also get $\hat{g}_{+}=\check{g}_{+}$.

(b) By definition there exists a $\mathbb{k}$-superscheme epimorphism $\Theta: \mathbb{A}_{\mathbb{k}}^{0 \mid d_{-}} \longrightarrow G_{-}^{<}$ which is given on every single $A \in(\mathrm{salg})_{\mathbb{k}}$ by

$$
\Theta_{A}: \mathbb{A}_{\mathbb{k}}^{0 \mid d_{-}}(A):=A_{1}^{\times d_{-}} \longrightarrow G_{-}^{<}(A), \quad\left(\eta_{i}\right)_{i \in I} \mapsto \Theta_{A}\left(\left(\eta_{i}\right)_{i \in I}\right):=\prod_{i \in I}\left(1+\eta_{i} Y_{i}\right) .
$$

We prove now that all these $\Theta_{A}$ 's are injective, so that $\Theta$ is indeed an isomorphism.

Let $\left(\hat{\eta}_{i}\right)_{i \in I},\left(\check{\eta}_{i}\right)_{i \in I} \in A_{1}^{\times d_{-}}$be such that $\Theta_{A}\left(\left(\hat{\eta}_{i}\right)_{i \in I}\right)=\Theta_{A}\left(\left(\check{\eta}_{i}\right)_{i \in I}\right)$, that is, $\vec{\prod}_{i \in I}\left(1+\hat{\eta}_{i} Y_{i}\right)=\vec{\prod}_{i \in I}\left(1+\check{\eta}_{i} Y_{i}\right)$. Then we can replay the proof of claim $(a)$, now taking $\hat{g}_{+}:=1=: \check{g}_{+}$. The outcome will again be $\hat{\eta}_{i}=\check{\eta}_{i}$ for all $i \in I$, i.e., $\left(\hat{\eta}_{i}\right)_{i \in I}=\left(\check{\eta}_{i}\right)_{i \in I}$. 
The first key consequence of the previous results is the following

Corollary 4.2.12. The supergroup $\mathbb{k}$-functor $G_{\mathcal{P}}$ considered above is representable, hence it is an affine $\mathbb{k}$-supergroup.

Proof. By Proposition 4.2.11 one has an isomorphism $G_{\mathcal{P}} \cong G_{+} \times G_{-}^{<}$as functors, hence as $\mathbb{k}$-superschemes. As $G_{+}$is representable by assumption, and $G_{-}^{<} \cong \mathbb{A}_{\mathbb{k}}^{0 \mid d_{-}}$ is representable too (by Proposition 4.2.11(b) above), we get that $G_{\mathcal{P}} \cong G_{+} \times G_{-}^{<}$ is representable as well.

With the next step we fix some further details, so to see that the assignment $\mathcal{P} \mapsto G_{\mathcal{P}}$ eventually yields a functor of the type we are looking for.

Proposition 4.2.13. For every $\mathcal{P} \in(\mathrm{lsHCp})_{\mathbb{k}}$, let $G_{\mathcal{P}}$ be defined as above. Then:

(a) $G_{\mathcal{P}}$ is globally strongly split;

(b) the defining embedding of $G_{\mathcal{P}}$ inside $\mathrm{GL}(V)$ is closed, so that $G_{\mathcal{P}}$ identifies with a closed subgroup of $\mathrm{GL}(V)$;

(c) the above construction of $G_{\mathcal{P}}$ naturally extends to morphisms, so to provide a functor $\Psi_{\ell}:(\text { lsHCp })_{\mathbb{k}} \longrightarrow$ (lgss-fsgroups $)_{\mathbb{k}}$.

Proof. (a) We already noticed that, by the very construction, one has $\left(G_{\mathcal{P}}\right)_{\mathbf{0}}=$ $G_{+}$; this together with $G_{-}^{<} \cong \mathbb{A}_{\mathbb{k}}^{0 \mid d_{-}}$yields $G_{\mathcal{P}} \cong G_{+} \times G_{-}^{<} \cong G_{+} \times \mathbb{A}_{\mathbb{k}^{0 \mid}}^{0 \mid d_{-}}$(see above). Furthermore, by construction and Lemma 4.2.6 (b) we have that $G_{-}^{<} \cong$ $\mathbb{A}_{\mathrm{k}}^{0 \mid d_{-}}$is stable by the adjoint action of $\left(G_{\mathcal{P}}\right)_{\mathbf{0}}=G_{+}$. Eventually, all this means that $G_{\mathcal{P}}$ is globally strongly split.

(b) Due to Proposition 4.2.7 and Proposition 4.2.11, it is enough to prove that both $G_{+}$and $G_{-}^{<}$are closed subsuperschemes in GL $(V)$. The first property holds by the definition of an lsHCp, so we are left to cope with the second.

In the proof of Proposition 4.2.11 ( $b$ ) we saw that there exists a $\mathbb{k}$-superscheme isomorphism $\Theta: \mathbb{A}_{\mathbb{k}^{0}}^{0 \mid d_{-}} \longrightarrow G_{-}^{<}$given by

$$
\left(\eta_{i}\right)_{i \in I} \mapsto \Theta_{A}\left(\left(\eta_{i}\right)_{i \in I}\right):=\prod_{i \in I}\left(1+\eta_{i} Y_{i}\right)=: \mathcal{Y} \quad \text { for any } A \in(\mathrm{salg})_{\mathbb{K}} .
$$

Expanding the last product — inside $\operatorname{End}_{\mathbb{k}}(V)(A)$, say — yields

$$
\mathcal{Y}:=\Theta_{A}\left(\left(\eta_{i}\right)_{i \in I}\right)=1+\sum_{k=1}^{d_{-}} \prod_{i_{1}<\cdots<i_{k}} \eta_{i_{1}} \cdots \eta_{i_{k}} Y_{i_{1}} \cdots Y_{i_{k}}=\mathcal{Y}_{0}^{\prime}+\mathcal{Y}_{1}^{\prime}
$$

where we set $\mathcal{Y}_{0}^{\prime}:=\sum_{k \text { even }}^{d_{-}}(-1)^{\left(\begin{array}{c}k \\ 2\end{array}\right)} \prod_{i_{1}<\cdots<i_{k}} \eta_{i_{1}} \cdots \eta_{i_{k}} Y_{i_{1}} \cdots Y_{i_{k}} \in \operatorname{End}_{\mathbb{k}}(V)_{\mathbf{0}}(A)$ and similarly $\mathcal{Y}_{1}^{\prime}:=\sum_{k \text { odd }}^{d_{-}}(-1)^{\left(\begin{array}{c}k-1 \\ 2\end{array}\right)} \prod_{i_{1}<\cdots<i_{k}} \eta_{i_{1}} \cdots \eta_{i_{k}} Y_{i_{1}} \cdots Y_{i_{k}} \in \operatorname{End}_{\mathbb{k}}(V)_{1}(A)$.

Now recall that $\mathfrak{g l}(V)$ is $\mathbb{k}$-free and $\mathfrak{g l}(V)_{\mathbf{1}}=\mathfrak{g}_{\mathbf{1}} \oplus \mathfrak{q}$ with both $\mathfrak{g}_{\mathbf{1}}$ and $\mathfrak{q}$ being $\mathbb{k}$-free. Then the given expansions of $\mathcal{Y}_{0}^{\prime}$ and $\mathcal{Y}_{1}^{\prime}$ prove that, with respect to some $\mathbb{k}^{-}$ basis $B$ of $\operatorname{End}_{\mathbb{k}}(V)=\mathfrak{g l}(V)$ extending that of $\mathfrak{g}_{1}$ given by the $Y_{i}$ 's, the coefficients $c_{b}(b \in B)$ of both $\mathcal{Y}_{0}^{\prime}$ and $\mathcal{Y}_{1}^{\prime}$ are polynomials in the $\eta_{j}$ 's. In particular, the coefficients in $\mathcal{Y}_{1}^{\prime}$ of each basis element $Y_{i}$ is of the form $\eta_{i}+\mathcal{O}_{i}(3)$, where $\mathcal{O}_{i}(3)$ is some polynomial in the $\eta_{j}$ 's in which only monomials of degree odd and at least 3 can

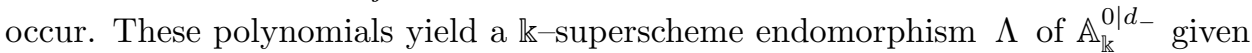
on $A$-points by $\Lambda_{A}:\left(\eta_{i}\right)_{i=1, \ldots, d_{-}} \mapsto\left(\eta_{i}+\mathcal{O}_{i}(3)\right)_{i=1, \ldots, d_{-}}$which is automatically an isomorphism (exploiting the fact that the variables $\eta_{i}$ are nilpotent). Setting 
$\widetilde{\eta}_{i}:=\Lambda_{A}^{-1}\left(\eta_{i}\right)$, all this implies that the previously mentioned coefficients $c_{b}(b \in B)$ are also polynomials in the $\widetilde{\eta}_{j}$ 's, say $c_{b}=P_{b}\left(\left\{\widetilde{\eta}_{j}\right\}_{j=1, \ldots, d_{-}}\right)$; in particular when $b=Y_{i}$ (for some $i$ ) we have $c_{i}:=c_{Y_{i}}=\widetilde{\eta}_{i}$. Therefore, our $G_{-}^{<}$is the set of zeroes (in the superscheme-theoretical sense) of the ideal $\left(\left\{c_{b}-P_{b}\left(\left\{c_{i}\right\}_{i=1, \ldots, d_{-}}\right)\right\}_{b \in B}\right)$ of the $\mathbb{k}$-algebra $\mathcal{O}\left(\operatorname{End}_{\mathbb{k}}(V)\right)$. Here we think of the $c_{b}$ 's as being elements of $\mathcal{O}\left(\operatorname{End}_{\mathbb{k}}(V)\right)$, which clearly makes sense in that they are defined as "coordinate functions". Therefore $G_{-}^{<}$is closed in $\operatorname{End}_{\mathbb{k}}(V)$, hence also in $\operatorname{GL}(V)$, so that it is a closed $\mathbb{k}$-subsuperscheme of $\operatorname{GL}(V)$.

(c) The previous claims ensure that $G_{\mathcal{P}}$ is a $\mathbb{k}$-supergroup, actually a linear one; moreover, we also remarked that $\left(G_{\mathcal{P}}\right)_{0}=G_{+}$. In addition, again by the very construction and by Proposition 4.2 .11 we find that $\operatorname{Lie}\left(G_{\mathcal{P}}\right)=\mathfrak{g}$. In particular, by the assumptions on $\mathfrak{g}$ this implies that the supergroup $G_{\mathcal{P}}$ is fine. Overall this means that $G_{\mathcal{P}} \in(\text { lgss-fsgroups })_{\mathbb{K}}$.

In order to have a functor $\Psi_{\ell}:(\operatorname{lsHCp})_{\mathbb{k}} \longrightarrow(\text { lgss-fsgroups })_{\mathbb{k}}$ we still need to define $\Psi_{\ell}$ on morphisms of $(\mathrm{lsHCp})_{\mathbb{k}}$. Letting $\left(\Omega_{+}, \omega\right): \mathcal{P}^{\prime}:=\left(G_{+}^{\prime}, \mathfrak{g}^{\prime}\right) \longrightarrow$ $\left(G_{+}^{\prime \prime}, \mathfrak{g}^{\prime \prime}\right)=: \mathcal{P}^{\prime \prime}$ be a morphism in $\left(\mathrm{lsHCp}_{\mathbb{k}}\right.$, we define $\Psi_{\ell}\left(\left(\Omega_{+}, \omega\right)\right)$ on $A$-points - for any $A \in(\mathrm{salg})_{\mathbb{k}}$ - as follows. Given $g^{\prime} \in \Psi_{\ell}\left(\mathcal{P}^{\prime}\right)$, let

$$
g^{\prime}=g_{+}^{\prime} \cdot \prod_{i \in I^{\prime}}\left(1+\eta_{i}^{\prime} Y_{i}\right)
$$

be its unique factorization after the factorization $G_{\mathcal{P}^{\prime}}=G_{+}^{\prime} \times G_{-}^{\prime}$ of $G_{\mathcal{P}^{\prime}}:=\Psi_{\ell}\left(\mathcal{P}^{\prime}\right)$ as in Proposition 4.2.11(a). Then set

$$
\Psi_{\ell}\left(\left(\Omega_{+}, \omega\right)\right)_{A}\left(g^{\prime}\right):=\Omega_{+}\left(g_{+}^{\prime}\right) \cdot \prod_{i \in I^{\prime}}\left(1+\eta_{i}^{\prime} \omega\left(Y_{i}\right)\right) .
$$

It is then a bookkeeping matter to check that this map is actually a group morphism, and that all properties required for that to yield a functor, as desired, are indeed satisfied.

In the end, our main result is that the $\Psi_{\ell}$ above is a quasi-inverse such as we were looking for.

Theorem 4.2.14. The functor $\Psi_{\ell}:(\operatorname{lsHCp})_{\mathbb{k}} \longrightarrow(\text { lgss-fsgroups })_{\mathbb{k}}$ is inverse, up to a natural isomorphism, to the functor $\Phi_{\ell}$ : (lgss-fsgroups $)_{\mathbb{k}} \longrightarrow(\operatorname{lsHCp})_{\mathbb{k}}$. In other words, these two are category equivalences, quasi-inverse to each other.

Proof. The previous results altogether show that, for any $\mathcal{P} \in(\mathrm{lsHCp})_{\mathbb{k}}$, the sHCp associated with $\Psi_{\ell}(\mathcal{P}):=G_{\mathcal{P}}$ is nothing but $\mathcal{P}$ itself, up to isomorphism. In other words, we have $\Phi_{\ell}\left(\Psi_{\ell}(\mathcal{P})\right)=\Phi_{\ell}\left(G_{\mathcal{P}}\right) \cong \mathcal{P}$. Moreover, tracking the whole construction one realizes at once that it is natural, i.e., all these isomorphisms match together to give $\Phi_{\ell} \circ \Psi_{\ell} \cong I d_{(\mathrm{lsHCp})_{\mathrm{k}}}$.

As to the composition $\Psi_{\ell} \circ \Phi_{\ell}$, let $G \in$ (lgss-fsgroups) $)_{\mathbb{k}}$ and $\mathcal{P}:=\Phi_{\ell}(G)=$ $\left(G_{\mathbf{0}}, \mathfrak{g}\right) \in(\mathrm{lsHCp})_{\mathbb{k}}$ - with $\mathfrak{g}=\operatorname{Lie}(G)-$ and $G_{\mathcal{P}}:=\Psi_{\ell}(\mathcal{P})=\Psi_{\ell}\left(\Phi_{\ell}(G)\right)$. Clearly, everything amounts to proving that $G_{\mathcal{P}}=G$ - as closed subgroups inside $\mathrm{GL}(V)$, with $V$ as in Definition 4.2.1 (a) above. Note that the inclusion $G_{\mathcal{P}} \subseteq G$ holds true by construction, since all generators of $G_{\mathcal{P}}(A)$ belong to $G(A)$.

First, by Proposition 4.2.13(b) we have that $G_{\mathcal{P}}$ is a closed subgroup of $\mathrm{GL}(V)$; the same holds for $G$, by assumption. As $G_{\mathcal{P}} \subseteq G$, we can argue that $G_{\mathcal{P}}$ is closed also inside $G$. 
Then we apply Theorem 3.2 .13 to $H:=G_{\mathcal{P}}$ and $K:=G$, finding global splittings $\left(G_{\mathcal{P}}\right)_{\mathbf{0}} \times\left(G_{\mathcal{P}}\right)_{\mathbf{1}} \cong G_{\mathcal{P}}$ and $G_{\mathbf{0}} \times G_{\mathbf{1}} \cong G$ which are consistent with each other, as in the cited theorem, in particular $\left(G_{\mathcal{P}}\right)_{0} \subseteq G_{\mathbf{0}}$ and $\left(G_{\mathcal{P}}\right)_{1} \subseteq G_{1}$.

Third, the inclusion $\left(G_{\mathcal{P}}\right)_{0} \subseteq G_{0}$ is an identity by the very construction of $G_{\mathcal{P}}$. So we are only left to prove that the inclusion $\left(G_{\mathcal{P}}\right)_{1} \subseteq G_{1}$, provided in the second step above, is an equality too.

Now, the fact that the splittings of $G_{\mathcal{p}}$ and $G$ are compatible is equivalent to the fact that the projection

$$
\pi: \mathcal{O}(G)=\mathcal{O}\left(G_{\mathbf{0}}\right) \otimes \mathcal{O}\left(G_{\mathbf{1}}\right) \longrightarrow \mathcal{O}\left(\left(G_{\mathcal{P}}\right)_{\mathbf{0}}\right) \otimes \mathcal{O}\left(\left(G_{\mathcal{P}}\right)_{\mathbf{1}}\right)=\mathcal{O}\left(G_{\mathcal{P}}\right)
$$

is of the form $\pi=\pi_{\mathbf{0}} \otimes \pi_{\mathbf{1}}$, where $\pi_{\mathbf{0}}: \mathcal{O}\left(G_{\mathbf{0}}\right):=\mathcal{O}\left(G_{\text {ev }}\right) \longrightarrow \mathcal{O}\left(\left(G_{\mathcal{p}}\right)_{\text {ev }}\right)=$ : $\mathcal{O}\left(\left(G_{\mathcal{P}}\right)_{0}\right)$ is canonically defined and $\pi_{\mathbf{1}}: \mathcal{O}\left(G_{\mathbf{1}}\right) \longrightarrow \mathcal{O}\left(\left(G_{\mathcal{P}}\right)_{1}\right)$ is a suitable morphism. Now, by construction $\mathcal{O}\left(G_{1}\right)$ is a Grassmann algebra, namely $\mathcal{O}\left(G_{\mathbf{1}}\right)=\bigwedge \operatorname{Lie}\left(G_{\mathbf{1}}\right)^{*}$, and $\mathcal{O}\left(G_{\mathbf{1}}\right)=\bigwedge \operatorname{Lie}\left(\left(G_{\mathcal{P}}\right)_{\mathbf{1}}\right)^{*}$ by similar reasons. But still by construction we have $\operatorname{Lie}\left(G_{1}\right)=\mathfrak{g}_{1}=\operatorname{Lie}\left(\left(G_{\mathcal{P}}\right)_{1}\right)$ inside $\operatorname{Lie}(\operatorname{GL}(V))=\mathfrak{g l}(V)$ so the inclusion map $\operatorname{Lie}\left(\left(G_{\mathcal{P}}\right)_{1}\right)=\bigwedge \mathfrak{g}_{1} \longleftrightarrow \bigwedge \mathfrak{g}_{1}=\operatorname{Lie}\left(G_{1}\right)$ is just the identity; hence its dual, namely the projection map $\pi_{\mathbf{1}}: \mathcal{O}\left(G_{1}\right) \longrightarrow \mathcal{O}\left(\left(G_{\mathcal{P}}\right)_{1}\right)$, is the identity from $\mathcal{O}\left(G_{1}\right)=\bigwedge \mathfrak{g}_{1}^{*}$ to $\mathcal{O}\left(\left(G_{\mathcal{P}}\right)_{1}\right)=\bigwedge \mathfrak{g}_{1}^{*}$. In turn, the inclusion $\left(G_{\mathcal{P}}\right)_{\mathbf{1}} \subseteq G_{\mathbf{1}}$ is necessarily the identity too.

4.3. The converse functor: General case. We shall now face the task of providing a quasi-inverse to the functor $\Phi$ : (fsgroups $)_{\mathbb{k}} \longrightarrow(\mathrm{sHCp})_{\mathbb{k}}$ in greater generality. In the end, it will turn out that this will be successful only if we bound ourselves to deal with fine supergroups which are globally strongly split. In other words, a fine supergroup $G$ can be "reconstructed" starting from its associated sHCp if

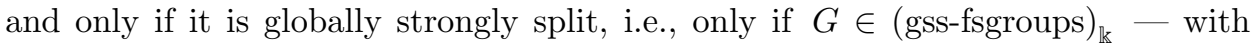
notation of Definition 3.2.6. Therefore, for sheer notational purposes we introduce the following

Definition 4.3.1. We denote by $\Phi_{g}:$ (gss-fsgroups $)_{\mathbb{k}} \longrightarrow(\mathrm{sHCp})_{\mathbb{k}}$ the restriction to the subcategory (gss-fsgroups $)_{\mathbb{k}}$ of the functor $\Phi:(\text { fsgroups })_{\mathbb{k}} \longrightarrow(\mathrm{sHCp})_{\mathbb{k}_{\mathbb{k}}}$ considered in Proposition 4.1.3.

By the way, note that by $\operatorname{Remark} 3.2 .7$ if $\operatorname{Lie}(G)$ is representable for a supergroup $G$, then asking $G$ to be fine and gs-split actually amounts to asking that $G$ be gssplit only.

We are ready to go and construct a quasi-inverse functor to $\Phi_{g}$. As we shall presently see, the very construction is modeled on that of $\Phi_{\ell}$, and also many arguments used in the proofs are essentially the same, up to technical modifications. The key difference with the linear case is the following. Roughly speaking, in that setup having an embedding of $\mathcal{P}$ inside $\mathfrak{g l}(V)$ allowed us to construct $G_{\mathcal{P}}$ as a subsupergroup of $\mathrm{GL}(V)$. Also, we could investigate the properties of such a group, hence proving all our results, just exploiting this "native" embedding of $G_{\mathcal{P}}$ into $\mathrm{GL}(V)$ and then into $\operatorname{End}_{\mathbb{k}}(V)$ too. In the general case such a linearization is not available; nevertheless, we can achieve a "partial linearization", which will still be enough for our purposes.

Indeed, first we construct our candidate for $G_{\mathcal{P}}$ by bare hands, in the form of a $\mathbb{k}$-supergroup functor. Then we find a suitable representation of $\mathcal{P}$, and we show that this will naturally "integrate" to a representation of $G_{\mathcal{P}}$. This representation, 
though not faithful, is still "faithful enough" to make it possible to apply again the arguments we used in the linear case. Thus we can replicate, mutatis mutandis, the process we followed in that case, and eventually find that our candidate for $G_{\mathcal{P}}$ actually does the job, namely $\mathcal{P} \mapsto G_{\mathcal{P}}$ yields the converse functor we were looking for.

As a first step, we start with the definition of $G_{\mathcal{P}}$.

Definition 4.3.2. Let $\mathcal{P}:=\left(G_{+}, \mathfrak{g}\right) \in(\mathrm{sHCp})_{\mathbb{k}}$ be an sHCp over $\mathbb{k}$. We fix in $\mathfrak{g}_{\mathbf{1}}$, which is $\mathbb{k}$-free, a $\mathbb{k}$-basis $\left\{Y_{i}\right\}_{i \in I}$ (for some index set $I$ ) and a total order in $I$.

(a) We introduce a $\mathbb{k}$-supergroup functor $G_{\mathcal{P}}:(\mathrm{salg})_{\mathbb{k}} \longrightarrow$ (groups) as follows. For any given $A \in(\mathrm{salg})_{\mathbb{k}}$, consider a formal element $\left(1+\eta_{i} Y_{i}\right)$ for each pair $\left(i, \eta_{i}\right) \in I \times A_{1}$.

We define $G_{\mathcal{P}}(A)$ by generators and relations: the set of generators is

$$
\begin{aligned}
\Gamma_{A}:=\left\{g_{+},\left(1+\eta_{i} Y_{i}\right) \mid g_{+} \in G_{+}(A),\right. & \left.\left(i, \eta_{i}\right) \in I \times A_{\mathbf{1}}\right\} \\
& =G_{+}(A) \cup\left\{\left(1+\eta_{i} Y_{i}\right)\right\}_{\left(i, \eta_{i}\right) \in I \times A_{\mathbf{1}}}
\end{aligned}
$$

(where $G_{+}(A):=G_{+}\left(A_{0}\right)$, by abuse of notation) and the set of relations is

$$
\begin{gathered}
g_{+}^{\prime} \cdot g_{+}^{\prime \prime}=g_{+\dot{G}_{+}}^{\prime} g_{+}^{\prime \prime} \quad \forall g_{+}^{\prime}, g_{+}^{\prime \prime} \in G_{+}(A), \\
\left(1+\eta_{i} Y_{i}\right) \cdot g_{+}=g_{+} \cdot\left(1+c_{j_{1}} \eta_{i} Y_{j_{1}}\right) \cdots\left(1+c_{j_{k}} \eta_{i} Y_{j_{k}}\right) \\
\forall\left(i, \eta_{i}\right) \in I \times A_{\mathbf{1}}, g_{+} \in G_{+}(A), \quad \text { with } \quad A d\left(g_{+}^{-1}\right)\left(Y_{i}\right)=c_{j_{1}} Y_{j_{1}}+\cdots+c_{j_{k}} Y_{j_{k}}, \\
\left(1+\eta_{i}^{\prime} Y_{i}\right) \cdot\left(1+\eta_{i}^{\prime \prime} Y_{i}\right)=\left(1_{G_{+}}+\eta_{i}^{\prime \prime} \eta_{i}^{\prime} Y_{i}^{\langle 2\rangle}\right)_{G_{+}} \cdot\left(1+\left(\eta_{i}^{\prime}+\eta_{i}^{\prime \prime}\right) Y_{i}\right) \quad \forall i \in I, \\
\left(1+\eta_{j} Y_{j}\right) \cdot\left(1+\eta_{i} Y_{i}\right)=\left(1_{G_{+}}+\eta_{i} \eta_{j}\left[Y_{i}, Y_{j}\right]\right)_{G_{+}} \cdot\left(1+\eta_{i} Y_{i}\right) \cdot\left(1+\eta_{j} Y_{j}\right) \quad \forall j>i(\in I), \\
\left(1+0_{A} Y_{i}\right)=1 \\
\forall i \in I
\end{gathered}
$$

where the first line just means that for generators chosen in $G_{+}(A)$ their product, denoted with ".", inside $G_{\mathcal{P}}(A)$ is the same as in $G_{+}(A)$, where it is denoted with " $\dot{G}_{+}$"; moreover, notation like $\left(1_{G_{+}}+\eta_{i}^{\prime \prime} \eta_{i}^{\prime} Y_{i}^{\langle 2\rangle}\right)_{G_{+}}$and $\left(1_{G_{+}}+\eta_{i} \eta_{j}\left[Y_{i}, Y_{j}\right]\right)_{G_{+}}$ denotes two well-defined elements in $G_{+}(A)$ - see the proof of Lemma 4.2.6 for a reminder - that in the sequel we shall denote more simply as $\left(1+\eta_{i}^{\prime \prime} \eta_{i}^{\prime} Y_{i}^{\langle 2\rangle}\right)$ and $\left(1+\eta_{i} \eta_{j}\left[Y_{i}, Y_{j}\right]\right)$.

This yields the functor $G_{\mathcal{P}}$ on objects, and one then defines it on morphisms in the obvious way.

(b) We define a $\mathbb{k}$-functor $G_{-}^{<}:(\mathrm{salg})_{\mathbb{k}} \longrightarrow($ sets $)$ as follows. For $A \in(\mathrm{salg})_{\mathbb{k}}$ we set

$$
G_{-}^{<}(A):=\left\{\overrightarrow{\prod_{i \in I}}\left(1+\eta_{i} Y_{i}\right) \mid \eta_{i} \in A_{\mathbf{1}} \forall i \in I\right\} \quad\left(\subseteq G_{\mathcal{P}}(A)\right)
$$

where $\vec{\prod}_{i \in I}$ denotes an ordered product (with respect to the fixed total order in $I)$. This defines the functor $G_{-}^{<}$on objects, and its definition on morphism is then the obvious one. 
Remarks 4.3.3.

(a) By its very definition $G_{-}^{<}$can be thought of as a subfunctor of $G_{\mathcal{P}}$.

(b) By definition both $G_{-}^{<}$and $G_{\mathcal{P}}$ depend on the choice of the ordered $\mathbb{k}^{-}$ basis $\left\{Y_{i}\right\}_{i \in I}$ of $\mathfrak{g}_{\mathbf{1}}$; nevertheless, basing on remark (c) here below one can easily show - by the same arguments used for Proposition 4.2.7 — that $G_{\mathcal{P}}$ is actually independent of this choice.

(c) Alternatively, one can modify the very definition of $G_{\mathcal{P}}$, giving a different presentation of it which is intrinsically independent of any choice of basis of $\mathfrak{g}_{\mathbf{1}}$, as it does not make use of any $\mathbb{k}$-basis $\left\{Y_{i}\right\}_{i \in I}$ of $\mathfrak{g}_{\mathbf{1}}$. Indeed, for each $A \in(\mathrm{salg})_{\mathbb{k}}$ one takes the group $G_{\mathcal{P}}^{\bullet}(A)$ with the (larger) set of generators

$$
\begin{aligned}
\Gamma_{A}^{\bullet}:=\left\{g_{+},(1+\eta Y) \mid g_{+} \in G_{+}(A),(Y, \eta)\right. & \left.\in \mathfrak{g}_{\mathbf{1}} \times A_{\mathbf{1}}\right\} \\
& =G_{+}(A) \cup\{(1+\eta Y)\}_{(Y, \eta) \in \mathfrak{g}_{1} \times A_{\mathbf{1}}}
\end{aligned}
$$

and (larger) set of relations (for $g_{+}^{\prime}, g_{+}^{\prime \prime} \in G_{+}(A), \eta, \eta^{\prime}, \eta^{\prime \prime} \in A_{\mathbf{1}}, Y, Y^{\prime}, Y^{\prime \prime} \in \mathfrak{g}_{\mathbf{1}}$ )

$$
\begin{gathered}
g_{+}^{\prime} \cdot g_{+}^{\prime \prime}=g_{+\dot{G}_{+}}^{\prime} g_{+}^{\prime \prime}, \quad(1+\eta Y) \cdot g_{+}=g_{+} \cdot\left(1+\eta \operatorname{Ad}\left(g_{+}^{-1}\right)(Y)\right), \\
\left(1+\eta^{\prime} Y\right) \cdot\left(1+\eta^{\prime \prime} Y\right)=\left(1_{G_{+}}+\eta^{\prime \prime} \eta^{\prime} Y^{\langle 2\rangle}\right)_{G_{+}} \cdot\left(1+\left(\eta^{\prime}+\eta^{\prime \prime}\right) Y\right), \\
\left(1+\eta^{\prime \prime} Y^{\prime \prime}\right) \cdot\left(1+\eta^{\prime} Y^{\prime}\right)=\left(1_{G_{+}}+\eta^{\prime} \eta^{\prime \prime}\left[Y^{\prime}, Y^{\prime \prime}\right]\right)_{G_{+}} \cdot\left(1+\eta^{\prime} Y^{\prime}\right) \cdot\left(1+\eta^{\prime \prime} Y^{\prime \prime}\right), \\
\left(1+\eta Y^{\prime}\right) \cdot\left(1+\eta Y^{\prime \prime}\right)=\left(1+\eta\left(Y^{\prime}+Y^{\prime \prime}\right)\right), \\
\left(1+\eta 0_{\mathfrak{g}_{1}}\right)=1, \quad\left(1+0_{A} Y\right)=1 .
\end{gathered}
$$

Here almost all relations are sheer generalizations of those in Definition 4.3.2 $(a)$, the exceptions being $\left(1+\eta 0_{\mathfrak{g}_{1}}\right)=1$ and $\left(1+\eta Y^{\prime}\right) \cdot\left(1+\eta Y^{\prime \prime}\right)=\left(1+\eta\left(Y^{\prime}+Y^{\prime \prime}\right)\right)$. In particular, the latter together with $(1+\eta Y) \cdot g_{+}=g_{+} \cdot\left(1+\eta \operatorname{Ad}\left(g_{+}^{-1}\right)(Y)\right)$ yields

$$
\left(1+\eta_{i} Y_{i}\right) \cdot g_{+}=g_{+} \cdot\left(1+c_{j_{1}} \eta_{i} Y_{j_{1}}\right) \cdots\left(1+c_{j_{k}} \eta_{i} Y_{j_{k}}\right)
$$

when $A d\left(g_{+}^{-1}\right)\left(Y_{i}\right)=c_{j_{1}} Y_{j_{1}}+\cdots+c_{j_{k}} Y_{j_{k}}$. Furthermore, again the relations of type $\left(1+\eta Y^{\prime}\right) \cdot\left(1+\eta Y^{\prime \prime}\right)=\left(1+\eta\left(Y^{\prime}+Y^{\prime \prime}\right)\right)$ imply that for $Y=\sum_{s=1}^{k} c_{j_{s}} Y_{j_{s}}$ we have

$$
(1+\eta Y)=\left(1+\eta \sum_{s=1}^{k} c_{j_{s}} Y_{j_{s}}\right)=\prod_{s=1}^{k}\left(1+c_{j_{s}} \eta Y_{j_{s}}\right)
$$

where the product actually can be done in any order, as the factors in it mutually commute; thus each generator $(1+\eta Y)$ can be obtained via the $\left(1+\eta_{i} Y_{i}\right)$ 's. This easily implies that mapping $g_{+}$and $\left(1+\eta_{i} Y_{i}\right)$ in $G_{\mathcal{P}}(A)$ respectively to $g_{+}$ and $\left(1+\eta_{i} Y_{i}\right)$ in $G_{\mathcal{P}}^{\bullet}(A)$ yields a well-defined epimorphism $\phi_{A}: G_{\mathcal{P}}(A) \longrightarrow G_{\mathcal{P}}^{\bullet}(A)$. Conversely, considering inside $G_{\mathcal{P}}(A)$ the elements $(1+\eta Y):=\prod_{s=1}^{k}\left(1+c_{j_{s}} \eta Y_{j_{s}}\right)$ - for each $Y=\sum_{s=1}^{k} c_{j_{s}} Y_{j_{s}} \in \mathfrak{g}_{1}$ - one easily sees that all relations considered above to define $G_{\mathcal{p}}^{\bullet}(A)$ also hold true inside $G_{\mathcal{P}}(A)$, as they follow from the defining relations of the latter group. This implies that there exists also an epimorphism $\psi_{A}: G_{\mathcal{P}}^{\bullet}(A) \longrightarrow G_{\mathcal{P}}(A)$ which is the inverse of $\phi_{A}$ above. The construction of $\phi_{A}$ and $\psi_{A}$ is natural in $A$, so in the end $G_{\mathcal{P}}$ and $G_{\mathcal{P}}^{\bullet}$ are isomorphic as group functors.

Our goal is to show that assigning to each $\mathcal{P}$ its corresponding $G_{\mathcal{P}}$ one eventually gets a functor $\Psi_{g}:(\mathrm{sHCp})_{\mathbb{k}} \longrightarrow(\text { gss-fsgroups })_{\mathbb{k}}$ and also that such a functor is an 
equivalence, quasi-inverse to $\Phi_{g}:(\text { gss-fsgroups })_{\mathbb{k}} \longrightarrow(\mathrm{sHCp})_{\mathbb{k}}$. We shall achieve this result in several steps.

4.3.4. The representation $G_{\mathcal{P}} \longrightarrow \mathrm{GL}(V)$. Let $\mathfrak{g}=\mathfrak{g}_{0} \oplus \mathfrak{g}_{1}$ be our given Lie superalgebra, for which $\mathfrak{g}_{1}$ is $\mathbb{k}$-free of finite rank (see Definition 4.1.2). Hence we can fix a $\mathbb{k}$-basis $\left\{Y_{i}\right\}_{i \in I}$ of it, where $I$ is some finite index set in which we fix some total order. Recall that the universal enveloping algebra $U(\mathfrak{g})$ is given by $U(\mathfrak{g}):=T(\mathfrak{g}) / J$ where $T(\mathfrak{g})$ is the tensor algebra of $\mathfrak{g}$ and $J$ is the two-sided ideal in $T(\mathfrak{g})$ generated by the set

$$
\left\{x y-(-1)^{|x||y|} y x-[x, y], z^{2}-z^{\langle 2\rangle} \mid x, y \in \mathfrak{g}_{\mathbf{0}} \cup \mathfrak{g}_{\mathbf{1}}, z \in \mathfrak{g}_{\mathbf{1}}\right\} .
$$

It is known then - see for instance [22, $\S 7.2$, with the few, obvious changes needed to take into account the relations of type $z^{2}-z^{22\rangle}=0$ (that are superfluous in the setting therein) — that one has splitting(s) of $\mathbb{k}$-supermodules (actually, even of $\mathbb{k}$-supercoalgebras)

$$
U(\mathfrak{g})=U\left(\mathfrak{g}_{0}\right) \otimes_{\mathbb{k}} \wedge \mathfrak{g}_{1} \cong \bigwedge \mathfrak{g}_{1} \otimes_{\mathbb{k}} U\left(\mathfrak{g}_{0}\right) .
$$

In addition, by the freeness assumption on $\mathfrak{g}_{1}$ and our choice of a basis for it we have that $\bigwedge \mathfrak{g}_{1}$ is $\mathbb{k}$-free too, with $\mathbb{k}$-basis $\left\{Y_{i_{1}} Y_{i_{2}} \cdots Y_{i_{s}}|s \leq| I \mid, i_{1}<i_{2}<\cdots<i_{s}\right\}$ - hereafter, we drop the sign " $\wedge$ " to denote the product in $\Lambda \mathfrak{g}_{\mathbf{1}}$.

Now let $\mathbb{1}$ be the (one-dimensional) trivial representation of $\mathfrak{g}_{\mathbf{0}}$. Then by the standard process of induction from $\mathfrak{g}_{0}$ to $\mathfrak{g}$ - the former being thought of as a Lie subsuperalgebra of the latter - we can consider the induced representation $V:=\operatorname{Ind}_{\mathfrak{g}_{0}}^{\mathfrak{g}}(\mathbb{1})$. Looking at $\mathbb{1}$ and $V$ respectively as a module over $U\left(\mathfrak{g}_{0}\right)$ and over $U(\mathfrak{g})$, taking (4.7) into account we get

$$
V:=\operatorname{Ind}_{\mathfrak{g}_{0}}^{\mathfrak{g}}(\mathbb{1})=U(\mathfrak{g}) \underset{U\left(\mathfrak{g}_{0}\right)}{\otimes} \mathbb{1}=\bigwedge \mathfrak{g}_{\mathbf{1}} \underset{\mathbb{k}}{\otimes} \mathbb{1} \cong \bigwedge \mathfrak{g}_{1}
$$

The last one above is a natural $\mathbb{k}$-module isomorphism, uniquely determined once a specific element $\underline{b} \in \mathbb{1}$ is fixed that forms a $\mathbb{k}$-basis of $\mathbb{1}$ itself; the isomorphism is $\omega \otimes \underline{b} \mapsto \omega$ for all $\omega \in \bigwedge \mathfrak{g}_{1}$.

This representation-theoretical construction and its outcome clearly give rise to similar functorial counterparts, for the Lie algebra valued $\mathbb{k}$-superfunctors $\mathcal{L}_{\mathfrak{g}_{\mathbf{0}}}$ and $\mathcal{L}_{\mathfrak{g}}$, as well as for the $\mathbb{k}$-superfunctors associated with $U\left(\mathfrak{g}_{\mathbf{0}}\right)$ and $U(\mathfrak{g})$, in the standard way.

On the other hand, recall that $\mathfrak{g}_{0}=\operatorname{Lie}\left(G_{+}\right)$, and clearly $\mathbb{1}$ is also the trivial representation for $G_{+}$, as a classical, affine $\mathbb{k}$-group scheme. Then, by construction and by (4.8), it is clear that the representation of $\mathfrak{g}$ on the space $V$ also induces a representation of the sHCp $\mathcal{P}=\left(G_{+}, \mathfrak{g}\right)$ on the same $V$. In other words $V$ itself bears also a structure of $\left(G_{+}, \mathfrak{g}\right)$-module, in the sense of Definition 4.2.1 $(b)$ - just drop the faithfulness requirement. For later use, we denote by $\left(\boldsymbol{r}_{+}, \rho\right):\left(G_{+}, \mathfrak{g}\right) \longrightarrow$ $\operatorname{End}_{\mathbb{k}}(V)$ the pair of representation maps $\boldsymbol{r}_{+}: G_{+} \longrightarrow \mathrm{GL}(V)$ and $\rho: \mathfrak{g} \longrightarrow \mathfrak{g l}(V)$ which encode this $\left(G_{+}, \mathfrak{g}\right)$-module structure on $V$. Moreover, we shall also use $\rho$ again to denote the representation map $\rho: U(\mathfrak{g}) \longrightarrow \operatorname{End}_{\mathbb{k}}(V)$ describing the $U(\mathfrak{g})$-module structure on $V$.

Our key step now is to remark that the above $\left(G_{+}, \mathfrak{g}\right)$-module structure on $V$ will actually "integrate" to a $G_{\mathcal{P}}$-module structure, in a natural way. 
Proposition 4.3.5. Retain notation as above for the $\left(G_{+}, \mathfrak{g}\right)$-module $V$. There exists a unique structure of a (left) $G_{\mathcal{P}}$-module onto $V$ which satisfies the following conditions: for every $A \in(\mathrm{salg})_{\mathbb{K}}$, the representation map $\boldsymbol{r}_{\mathcal{P}, A}: G_{\mathcal{P}}(A) \longrightarrow$ $\mathrm{GL}(V)(A)$ is given on generators of $G_{\mathcal{p}}(A)$ - namely, all $g_{+} \in G_{+}(A)$ and $\left(1+\eta_{i} Y_{i}\right)$ for $i \in I, \eta_{i} \in A_{\mathbf{1}}-b y$

$$
\boldsymbol{r}_{\mathcal{P}, A}\left(g_{+}\right):=\boldsymbol{r}_{+}\left(g_{+}\right), \quad \boldsymbol{r}_{\mathcal{P}, A}\left(1+\eta_{i} Y_{i}\right):=\rho\left(1+\eta_{i} Y_{i}\right)=i d_{V}+\eta_{i} \rho\left(Y_{i}\right)
$$

or, in other words, $g_{+} \cdot v:=\boldsymbol{r}_{+}\left(g_{+}\right)(v)$ and $\left(1+\eta_{i} Y_{i}\right) \cdot v:=\rho\left(1+\eta_{i} Y_{i}\right)(v)=$ $v+\eta_{i} \rho\left(Y_{i}\right)(v)$ for all $v \in V(A)$. In particular, this yields a morphism of $\mathbb{k}-$ supergroup functors $\boldsymbol{r}_{\mathcal{P}}: G_{\mathcal{P}} \longrightarrow \mathrm{GL}(V)$.

Proof. This is, essentially, a straightforward consequence of the whole construction, and of the very definition of $G_{\mathcal{P}}$. Indeed, by definition of representation for the sHCp $\mathcal{P}$ we see that the operators $\boldsymbol{r}_{\mathcal{P}, A}\left(g_{+}\right)$and $\boldsymbol{r}_{\mathcal{P}, A}\left(1+\eta_{i} Y_{i}\right)$ on $V$ - associated with the generators of $G_{\mathcal{P}}(A)$ - do satisfy all relations which, by Definition 4.3.2. are satisfied by the generators themselves. Thus they uniquely provide a welldefined group morphism $\boldsymbol{r}_{\mathcal{P}, A}: G_{\mathcal{P}}(A) \longrightarrow \mathrm{GL}(V)(A)$ as required. The construction is clearly functorial in $A$, whence the claim.

The representation $\boldsymbol{r}_{\mathcal{p}}$ of $G_{\mathcal{P}}$ on $V$ will play the role which in the linear case was played by the "intrinsic" representation $V$ yielding the embedding of $G_{\mathcal{P}}$ into $\mathrm{GL}(V)$. In that case the representation was faithful, by assumption; in the general setup it is not the case any more. Nevertheless, the next result ensures that this representation is still "faithful enough" to allow us, in a sense, to adapt to the general setup the arguments used for the linear one.

Lemma 4.3.6. Let $V$ be as above, and $A \in(\mathrm{salg})_{\mathbb{k}}$. For any

$$
\hat{g}_{-}:=\prod_{i \in I}\left(1+\hat{\eta}_{i} Y_{i}\right) \in \operatorname{GL}(V)(A) \quad \text { and } \quad \check{g}_{-}:=\prod_{i \in I}\left(1+\check{\eta}_{i} Y_{i}\right) \in \operatorname{GL}(V)(A),
$$

the following are equivalent:

(a) $\hat{\eta}_{i}=\check{\eta}_{i}$ for all $i \in I$;

(b) $\hat{g}_{-}=\check{g}_{-}$;

(c) $\hat{g}_{-} . v=\check{g}_{-} . v$ for all $v \in V$;

(d) $\hat{g}_{-} \cdot \underline{b}=\check{g}_{-} \cdot \underline{b}$, where $\underline{b} \in \mathbb{1}$ form a $\mathbb{k}$-basis of $\mathbb{1}$ - see the remark after

Proof. Clearly $(a) \Longrightarrow(b) \Longrightarrow(c) \Longrightarrow(d)$, thus we only need to prove that $(d) \Longrightarrow(a)$.

To avoid confusion, let us fix some additional notation. When we are describing $V$ as $V=\bigwedge \mathfrak{g}_{1} \cdot \underline{b} \cong \bigwedge \mathfrak{g}_{1}$, we write the elements of the $\mathbb{k}$-basis $\left\{Y_{i}\right\}_{i \in I}$ of $\mathfrak{g}_{\mathbf{1}}$ as $\bar{Y}_{i}$ instead of $Y_{i}$. Thus the $\mathbb{k}$-linear isomorphism $\Lambda \mathfrak{g}_{\mathbf{1}} \cdot \underline{b} \cong \bigwedge \mathfrak{g}_{\mathbf{1}}$ is given by $\left(Y_{i_{1}} Y_{i_{2}} \cdots Y_{i_{s}}\right) \cdot \underline{b} \mapsto \bar{Y}_{i_{1}} \bar{Y}_{i_{2}} \cdots \bar{Y}_{i_{s}}-$ for all $i_{1}<i_{2}<\cdots<i_{s}$. Now, in terms of these $\mathbb{k}$-bases the element $\hat{g}_{-} \cdot \underline{b} \in V=\bigwedge \mathfrak{g}_{1} \cdot \underline{b}$ can be rewritten (by construction) as

$$
\hat{g}_{-\cdot} \underline{b}=\overrightarrow{\prod_{i \in I}}\left(1+\hat{\eta}_{i} Y_{i}\right) \cdot \underline{b}=\left(1+\sum_{i \in I}^{\vec{b}} \hat{\eta}_{i} Y_{i}+\mathcal{O}(2)\right) \cdot \underline{b}=1+\sum_{i \in I} \hat{\eta}_{i} \bar{Y}_{i}+\overline{\mathcal{O}}(2) .
$$

Here above by $\left(1+\vec{\sum}_{i \in I} \hat{\eta}_{i} Y_{i}+\mathcal{O}(2)\right)$ we denote the expansion of the product $\prod_{i \in I}\left(1+\hat{\eta}_{i} Y_{i}\right)$ as an element of $U(\mathfrak{g})$, with $\mathcal{O}(2)$ which represents further summands 
of order at least 2 in the $\eta_{i}$ 's, using the $Y_{i}$ 's as basis elements of $\mathfrak{g}_{1}$. Then of course $\left(1+\vec{\sum}_{i \in I} \hat{\eta}_{i} \bar{Y}_{i}+\overline{\mathcal{O}}(2)\right)$ is the analogous object written in terms of the $\bar{Y}_{i}$ 's. Similarly, taking $\check{g}_{-}$instead of $\hat{g}_{-}$we find

$$
\check{g}_{-} \cdot \underline{b}=\vec{\prod}\left(1+\check{\eta}_{i} Y_{i}\right) \cdot \underline{b}=\left(1+\sum_{i \in I} \check{\eta}_{i} Y_{i}+\mathcal{O}(2)\right) \cdot \underline{b}=1+\sum_{i \in I} \check{\eta}_{i} \bar{Y}_{i}+\overline{\mathcal{O}}(2) .
$$

Then the identity $\hat{g}_{-} \cdot \underline{b}=\check{g}_{-} \cdot \underline{b}$ yields

$$
1+\sum_{i \in I} \hat{\eta}_{i} \bar{Y}_{i}+\overline{\mathcal{O}}(2)=1+\sum_{i \in I} \check{\eta}_{i} \bar{Y}_{i}+\overline{\mathcal{O}}(2)
$$

an identity in $A \otimes\left(\bigwedge \mathfrak{g}_{1}\right)$, which in turn implies $\hat{\eta}_{i}=\check{\eta}_{i}$ for all $i \in I$, like in the proof of Proposition 4.2.11.

Roughly speaking, the equivalence between claims (b) and (c) in the above lemma is sort of a "partial faithfulness" of the $G_{\mathcal{P}}$-module $V$. This is what we need for our next result.

\section{Proposition 4.3.7.}

(a) The restriction of group multiplication in $G_{\mathcal{P}}$ provides superscheme isomorphisms

$$
G_{+} \times G_{-}^{<} \cong G_{\mathcal{P}}, \quad G_{-}^{<} \times G_{+} \cong G_{\mathcal{P}} .
$$

Moreover, the group $G_{\mathcal{P}}(A)$ is independent of the choice of an ordered $\mathbb{k}$-basis $\left\{Y_{i}\right\}_{i \in I}$ of $\mathfrak{g}_{1}$ used for its definition; the same holds true for the whole functor $G_{\mathcal{P}}$. Similarly, the sets $G_{+}\left(A_{1}^{(2)}\right) G_{-}^{<}(A)$ and $G_{-}^{<}(A) G_{+}\left(A_{1}^{(2)}\right)-c f$. Section $2.1 .1-$ both coincide with the subgroup of $G_{\mathcal{P}}(A)$ generated by $G_{+}\left(A_{1}^{(2)}\right)$ and $G_{-}^{<}(A)$, and they are independent of the choice of an ordered $\mathbb{k}$-basis of $\mathfrak{g}_{\mathbf{1}}$.

(b) There exists a $\mathbb{k}$-superscheme isomorphism $\mathbb{A}_{\mathbb{k}^{0}}^{0 \mid d_{-}} \cong G_{-}^{<}$, with $d_{-}:=|I|=$ $\operatorname{dim}_{\mathbb{k}}\left(\mathfrak{g}_{1}\right)$, given on $A$-points by

$$
\mathbb{A}_{\mathbb{k}}^{0 \mid d_{-}}(A)=A_{1}^{d_{-}} \longrightarrow G_{-}^{<}(A), \quad\left(\eta_{i}\right)_{i \in I} \mapsto \prod_{i \in I}^{\rightarrow}\left(1+\eta_{i} Y_{i}\right)
$$

Proof. (a) The proof follows by the same arguments we used for Proposition 4.2.7 and Proposition 4.2.11 (a). Indeed, acting exactly like in the proof of Proposition 4.2.7 we see - working on $A$-points, for each $A \in(\mathrm{salg})_{\mathbb{k}}$ — that $G_{+} \cdot G_{-}^{<}=G_{\mathcal{P}}$, i.e., the multiplication in $G_{\mathcal{P}}$ maps $G_{+} \times G_{-}^{<}$onto $G_{\mathcal{P}}$ itself. Indeed, the point is that the arguments in the proof of Proposition 4.2.7 actually only make use of some commutation formulas among elements of $G_{+}(A)$ and elements of the form $\left(1+\eta_{i} Y_{i}\right)$. But exactly the same formulas do hold again in the present $G_{\mathcal{P}}$ we are dealing with now, by its very construction (see Definition 4.3.2); hence we can succesfully replicate the same procedure. The same strategy also proves that $G_{-}^{<} \cdot G_{+}=G_{\mathcal{P}}$, i.e., the multiplication map from $G_{-}^{<} \times G_{+}$to $G_{\mathcal{P}}$ is onto again.

After this, we can adapt the arguments used for Proposition 4.2.11) (a) to show that the multiplication map from $G_{-}^{<}(A) \times G_{+}(A)$ onto $G_{\mathcal{P}}(A)$ is also injective, for each $A \in(\mathrm{salg})_{\mathbb{k}}$, so we prove the claim about $G_{-}^{<} \times G_{+} \cong G_{\mathcal{P}}$; similarly for $G_{+} \times G_{-}^{<} \cong G_{\mathcal{P}}$. In this case the "adaptation" consists in applying Lemma 4.3.6.

Our goal amounts to showing the following: for any $A \in(\mathrm{salg})_{\mathbb{k}}$, if $\hat{g}_{-} \hat{g}_{+}=$ $\check{g}_{-} \check{g}_{+}$for $\hat{g}_{-}, \check{g}_{-} \in G_{-}^{<}(A), \hat{g}_{+}, \check{g}_{+} \in G_{+}(A)$, then $\hat{g}_{-}=\check{g}_{-}$and $\hat{g}_{+}=\check{g}_{+}$. Actually, the first identity implies the second one, thus we cope only with the former. From $\hat{g}_{-} \hat{g}_{+}=\check{g}_{-} \check{g}_{+}$we get $\left(\hat{g}_{-} \hat{g}_{+}\right) \cdot v=\left(\check{g}_{-} \check{g}_{+}\right) \cdot v$ for every $v \in V(A)$. 
But definitions yield $\left(\hat{g}_{-} \hat{g}_{+}\right) \cdot v=\hat{g}_{-} \cdot\left(\hat{g}_{+} \cdot v\right)=\hat{g}_{-} \cdot v$ and $\left(\check{g}_{-} \check{g}_{+}\right) \cdot v=\check{g}_{-} \cdot\left(\check{g}_{+} \cdot v\right)=$ $\check{g}_{-} . v$, hence $\left(\hat{g}_{-} \hat{g}_{+}\right) \cdot v=\left(\check{g}_{-} \check{g}_{+}\right) \cdot v$ reads also $\hat{g}_{-} \cdot v=\check{g}_{-} \cdot v$. Writing $\hat{g}_{-}=$ $\vec{\prod}_{i \in I}\left(1+\hat{\eta}_{i} Y_{i}\right)$ and $\check{g}_{-}=\vec{\prod}_{i \in I}\left(1+\check{\eta}_{i} Y_{i}\right)$, by Lemma 4.3.6 we find $\hat{g}_{-}=\check{g}_{-}$.

Moreover, $G_{\mathcal{P}}$ is independent of the choice of basis of $\mathfrak{g}_{1}$ because of Remarks $4.3 .3(b)-(c)$.

As to the last part of claim (a), it is proved again like in Proposition 4.2.7.

(b) By construction there exists a morphism $\mathbb{A}_{\mathbb{k}^{0}}^{0 \mid d_{-}} \cong G_{-}^{<}$of $\mathbb{k}^{-}$-superschemes given on $A$-points by $\mathbb{A}_{\mathbb{k}}^{0 \mid d_{-}}(A)=A_{1}^{d_{-}} \longrightarrow G_{-}^{<}(A),\left(\eta_{i}\right)_{i \in I} \mapsto \vec{\prod}_{i \in I}\left(1+\eta_{i} Y_{i}\right)$. By the very definition of $G_{-}^{<}$this is even onto. On the other hand, it is an isomorphism because on $A$-points it is injective too. Indeed, this follows directly from Lemma 4.3.6. namely by the equivalence of claims (a) and (b) therein.

Like in the linear case, the previous result yields the following, direct consequence:

Corollary 4.3.8. For every super Harish-Chandra pair $\mathcal{P} \in(\mathrm{sHCp})_{\mathbb{k}}$, the supergroup functor $G_{\mathcal{P}}$ given by Definition 4.3 .2 is representable, hence it is a ( $n$ affine) $\mathbb{k}$-supergroup indeed. More precisely, $G_{\mathcal{P}}$ is represented by a $\mathbb{k}$-superalgebra $\mathcal{O}\left(G_{\mathcal{P}}\right)$, with $\mathbb{k}$-algebra isomorphisms

$$
\mathcal{O}\left(G_{\mathcal{P}}\right) \cong \mathcal{O}\left(G_{+}\right) \otimes_{\mathbb{k}} \mathcal{O}\left(G_{-}^{<}\right) \cong \mathcal{O}\left(G_{+}\right) \otimes_{\mathbb{k}} \mathbb{k}\left[\left\{\xi_{i}\right\}_{i \in I}\right] .
$$

Indeed, $\mathcal{O}\left(G_{\mathcal{P}}\right)$ is a Hopf $\mathbb{k}$-superalgebra, and the above are isomorphisms of super counital left $\mathcal{O}\left(G_{+}\right)$-comodule algebras.

Proof. By Proposition 4.3.7 the $\mathbb{k}$-functor $G_{\mathcal{P}}$ is the direct product of the two $\mathbb{k}-$ superschemes $G_{+}$and $G_{-}^{<} \cong \mathbb{A}_{\mathrm{k}}^{0 \mid d_{-}}$, which both are representable as functors. Then $G_{\mathcal{P}}$ is representable as well, namely it is represented by

$$
\mathcal{O}\left(G_{\mathcal{P}}\right) \cong \mathcal{O}\left(G_{+}\right) \otimes_{\mathbb{k}} \mathcal{O}\left(G_{-}^{<}\right) \cong \mathcal{O}\left(G_{+}\right) \otimes_{\mathbb{k}} \mathbb{k}\left[\left\{\xi_{i}\right\}_{i \in I}\right] .
$$

Moreover, the unit element of $G_{\mathcal{P}}$ is the product of the unit in $G_{+}$and the unit in $G_{-}^{<}$- in both factorizations $G_{\mathcal{P}}=G_{+} \cdot G_{-}^{<}$and $G_{\mathcal{P}}=G_{-}^{<} \cdot G_{+}$, so the above isomorphisms are counit-preserving. Finally, using the factorization $G_{\mathcal{P}}=G_{+} \cdot G_{-}^{<}$ the left multiplication restricted to $G_{+}$corresponds to the left multiplication in the left hand factor $G_{+}$, whence the above isomorphisms also preserve the left $\mathcal{O}\left(G_{+}\right)$-coaction. The claim follows.

We still need to fix some details to see that the recipe $\mathcal{P} \mapsto G_{\mathcal{P}}$ in the end does provide a functor of the type we are looking for. This is the outcome of the next step.

Proposition 4.3.9. For every $\mathcal{P} \in(\mathrm{sHCp})_{\mathbb{k}}$, let $G_{\mathcal{P}}$ be defined as above. Then:

(a) $G_{\mathcal{P}}$ is fine and globally strongly split, in short $G_{\mathcal{P}} \in$ (gss-fsgroups)

(b) the above construction of $G_{\mathcal{P}}$ naturally extends to morphisms in $(\mathrm{sHCp})_{\mathbb{k}}$, so it yields a unique functor $\Psi_{g}:(\mathrm{sHCp})_{\mathbb{k}} \longrightarrow$ (gss-fsgroups) $)_{\mathbb{k}}$ given on objects by $\Psi_{g}(\mathcal{P}):=G_{\mathcal{P}}$.

Proof. (a) Directly from definitions one has that $\left(G_{\mathcal{P}}\right)_{0}:=\left(G_{\mathcal{P}}\right)_{e v}$ coincides with $G_{+}$. Together with Proposition 4.3.7 and Corollary 4.3.8 this implies that $G_{\mathcal{P}}$ is globally strongly split, a global splitting being the factorization $G_{+} \times G_{-}^{<} \cong G_{\mathcal{P}}$ given in Proposition 4.3.7 
In addition, from this factorization one sees - by bare hands computation, following the very definition of Lie $(G)$ given in Definition 2.3.1 — that

$$
\operatorname{Lie}\left(G_{\mathcal{P}}\right)=\operatorname{Lie}\left(G_{+} \times G_{-}^{<}\right)=\operatorname{Lie}\left(G_{+}\right) \oplus T_{e}\left(G_{-}^{<}\right)=\mathcal{L}_{\mathfrak{g}_{0}} \oplus \mathcal{L}_{\mathfrak{g}_{1}}=\mathcal{L}_{\mathfrak{g}},
$$

that is (identifying $\mathcal{L}_{\mathfrak{g}}$ with $\mathfrak{g}$ as usual), simply Lie $\left(G_{\mathcal{P}}\right)=\mathfrak{g}$, this being an identification as Lie $\mathbb{k}$-superalgebras. As $\mathfrak{g}_{1}$ is $\mathbb{k}$-free of finite rank, by assumption (see Definition 4.1.2), we conclude that $G_{\mathcal{P}}$ is fine, as required.

(b) This is trivial, directly from definitions.

We now have available a functor $\Psi_{g}:(\mathrm{sHCp})_{\mathbb{k}} \longrightarrow(\text { gss-fsgroups })_{\mathbb{k}}$ which is our candidate to be a quasi-inverse to $\Phi_{g}:$ (gss-fsgroups $)_{\mathbb{k}_{\mathrm{k}}} \longrightarrow(\mathrm{sHCp})_{\mathbb{k}}$.

We first need to establish some additional results. The first one is technical.

Lemma 4.3.10. Let $H=\bar{H} \otimes_{\mathbb{k}} \wedge W^{H}$ be a strongly split Hopf $\mathbb{k}$-superalgebra. Identify $\bar{H}$ with $\bar{H} \otimes_{\mathbb{k}} 1$ and $\wedge W^{H}$ with $1 \otimes_{\mathbb{k}} \wedge W^{H} ;$ also, for $K \in\left\{H, \bar{H}, \wedge W^{H}\right\}$ let $K^{+}:=\operatorname{Ker}\left(\left.\epsilon_{H}\right|_{K}\right)$. Then for each $\phi \in\left(\bigwedge W^{H}\right)_{\mathbf{1}}^{+}=\left(\bigwedge W^{H}\right)_{\mathbf{1}}$ we have (using Sweedler's like notation)

$$
\Delta(\phi)=\phi \otimes 1+1 \otimes \phi+\sum_{(\phi)^{+}} \phi_{(1)}^{+} \otimes \phi_{(2)}^{+}
$$

where $\left(\phi_{(1)}^{+}, \phi_{(2)}^{+}\right) \in\left(H_{\mathbf{1}}^{[2]} \times H_{\mathbf{1}}\right) \cup\left(H_{\mathbf{1}} \times \bar{H}^{+}\right) \cup\left(H_{\mathbf{1}} \times H_{\mathbf{1}}^{[2]}\right)$ - with notation as in Section 2.1.1.

Proof. Let us start with $n=1$. Since $\epsilon(\phi)=0$, we can always write $\Delta(\phi)$ in the form $\Delta(\phi)=\phi \otimes 1+1 \otimes \phi+\sum_{(\phi)} \phi_{(1)}^{+} \otimes \phi_{(2)}^{+}$with $\phi_{(1)}^{+}, \phi_{(2)}^{+} \in H^{+}$. After that, recall that the "strong splitting" $H=\bar{H} \otimes_{\mathbb{k}} \wedge W^{H}$ is an isomorphism as augmented algebras with a left $\bar{H}$-action. By the way these $\bar{H}$-actions are defined (see Section 3.1.7) we see that this means that $\Delta(\phi) \equiv 1 \otimes \phi \bmod \left(J_{H} \otimes_{\mathbb{k}} H\right)$; in turn, this implies that we can write

$$
\Delta(\phi)=\phi \otimes 1+1 \otimes \phi+\sum_{(\phi)^{+}} \phi_{(1)}^{+} \otimes \phi_{(2)}^{+} \quad \text { with } \quad \phi_{(1)}^{+} \in J_{H}, \phi_{(2)}^{+} \in H^{+} .
$$

By the way, note that $J_{H}=H_{1}^{[2]} \oplus H_{1} \subseteq H^{+}$and $H^{+}=\bar{H}^{+} \oplus J_{H}=\bar{H}^{+} \oplus H_{1}^{[2]} \oplus H_{\mathbf{1}}$.

Finally, as $\Delta$ is parity-preserving one has $|\Delta(\phi)|=|\phi|=\mathbf{1}$, thus $\left|\phi_{(1)}^{+}\right|+\left|\phi_{(2)}^{+}\right|=$ 1 too.

First assume $\left|\phi_{(1)}^{+}\right|=\mathbf{0}$; then we have $\left|\phi_{(2)}^{+}\right|=\mathbf{1}$, which means $\phi_{(2)}^{+} \in\left(H_{\mathbf{1}} \cap\right.$ $\left.H^{+}\right)=H_{\mathbf{1}}$. In addition, $\left|\phi_{(1)}^{+}\right|=\mathbf{0}$ means $\phi_{(1)}^{+} \in H_{\mathbf{0}}$, so $\phi_{(1)}^{+} \in\left(H_{\mathbf{0}} \cap J_{H}\right)=$ $\left(H_{\mathbf{0}} \cap\left(H_{\mathbf{1}}^{[2]} \oplus H_{1}\right)\right)=H_{\mathbf{1}}^{[2]}$.

Second, let $\left|\phi_{(1)}^{+}\right|=\mathbf{1}$; then $\left|\phi_{(2)}^{+}\right|=\mathbf{0}$, which means $\phi_{(2)}^{+} \in\left(H_{\mathbf{0}} \cap H^{+}\right)$, hence from the above remark $\phi_{(2)}^{+} \in\left(H_{\mathbf{0}} \cap H^{+}\right)=\left(H_{\mathbf{0}} \cap\left(\bar{H}^{+} \oplus H_{\mathbf{1}}^{[2]} \oplus H_{\mathbf{1}}\right)\right)=\bar{H}^{+} \oplus H_{\mathbf{1}}^{[2]}$. Eventually, we can split $\phi_{(2)}^{+} \in \bar{H}^{+} \oplus H_{1}^{[2]}$ into the sum of a term in $\bar{H}^{+}$plus another in $H_{1}^{[2]}$, getting a result as claimed.

The next three results concern a finer analysis of a gs-split fine supergroup.

Proposition 4.3.11. Given $G \in$ (gss-fsgroups) $)_{\mathbb{k}}$, a $\mathbb{k}$-basis $\left\{Y_{i}\right\}_{i \in I}$ of $\mathfrak{g}_{\mathbf{1}}$ and $A \in(\mathrm{salg})_{\mathbb{k}}$, consider in $G(A)$ the elements $\left(1+\eta_{i} Y_{i}\right)$ for all $\eta_{i} \in A_{\mathbf{1}}, i \in I$ as recalled in the proof of Lemma 4.2.6. Then $G(A)$ is generated by

$$
G_{\mathbf{0}}(A) \cup\left\{\left(1+\eta_{i} Y_{i}\right) \mid\left(i, \eta_{i}\right) \in I \times A_{\mathbf{1}}\right\} .
$$


Proof. As $G$ is globally strongly split the $\mathbb{k}$-superalgebra $\mathcal{O}(G)$ identifies, up to isomorphism, with $\overline{\mathcal{O}(G)} \otimes_{\mathbb{k}} \wedge W^{\mathcal{O}(G)}=\mathcal{O}\left(G_{\mathbf{0}}\right) \otimes_{\mathbb{k}} \mathcal{O}\left(G_{\mathbf{1}}\right)$, where $\bigwedge W^{\mathcal{O}(G)}=$ $\mathcal{O}\left(G_{\mathbf{1}}\right)$ in turn identifies with $\mathbb{k}\left[\left\{\xi_{i}\right\}_{i \in I}\right]$ - for some finite set $I$ such that $|I|=$ $r k_{\mathbb{k}}\left(\mathfrak{g}_{1}\right)-W^{H}$ with $\operatorname{Span}_{\mathbb{k}}\left(\left\{\xi_{i}\right\}_{i \in I}\right)$ and $\mathfrak{g}_{\mathbf{1}}$ with $\left(W^{H}\right)^{*}:=\operatorname{Hom}_{\mathbb{k}}\left(W^{H}, \mathbb{k}\right)$. Moreover, by definition the subgroup $G_{\mathbf{0}}$ of $G$ can be characterized as follows: for any $A \in(\mathrm{salg})_{\mathbb{k}}$ one has

$$
G_{\mathbf{0}}(A)=\left\{g \in \operatorname{Hom}_{\left(\operatorname{salg}_{\mathbf{k}_{\mathbf{k}}}\right.}\left(\mathcal{O}\left(G_{\mathbf{0}}\right) \otimes_{\mathbb{k}} \mathcal{O}\left(G_{1}\right), A\right)|g|_{1 \otimes_{\mathfrak{k}} \mathcal{O}\left(G_{1}\right)}=\left.\epsilon\right|_{1 \otimes_{\mathfrak{k}} \mathcal{O}\left(G_{1}\right)}\right\} .
$$

Recall - see the proof of Lemma 4.2.6 - that if $Z \in \mathfrak{g}$ and $e \in A$ are homogeneous of the same degree and $e^{2}=0$, then $(1+e Z) \in G(A)$; in particular this applies for any $Y:=Z \in \mathfrak{g}_{\mathbf{1}}$ and $\eta \in A_{\mathbf{1}}$, so that $(1+\eta Y) \in G(A)$. Hereafter $\eta Y \in A_{1} \mathfrak{g}_{1} \subseteq \mathfrak{g}(A)$ is thought of as the unique $A$-valued $\epsilon_{\mathcal{O}\left(G_{\mathbf{0}}\right)}$-derivation of $\mathbb{k}\left[\left\{\xi_{i}\right\}_{i \in I}\right]$ which maps every $\xi_{i}$ to $\eta Y\left(\xi_{i}\right)$ - through the $\mathbb{k}$-module identification $\mathfrak{g}_{\mathbf{1}}=\left(W^{H}\right)^{*}:=\operatorname{Hom}_{\mathbb{k}}\left(W^{H}, \mathbb{k}\right)=\operatorname{Hom}_{\mathbb{k}}\left(\operatorname{Span}_{\mathbb{k}}\left(\left\{\xi_{i}\right\}_{i \in I}\right), \mathbb{k}\right)$ mentioned above and acts onto $\mathcal{O}\left(G_{\mathbf{0}}\right)$ as the counit map $\epsilon_{\mathcal{O}\left(G_{\mathbf{0}}\right)}$.

Note that, since $\eta^{2}=0$, each morphism $(1+\eta Y)$ vanishes on $\left(\mathcal{O}(G)^{+}\right)^{2}$, hence in particular on $\left(\mathcal{O}(G)_{1}\right)^{2}$. Moreover, such a $(1+\eta Y)$ also vanishes on $\mathcal{O}\left(G_{\mathbf{0}}\right)^{+}$, by construction.

Let us now fix a (finite) $\mathbb{k}$-basis $\left\{Y_{i}\right\}_{i \in I}$ of $\mathfrak{g}_{\mathbf{1}}$; namely, we take the unique one for which $Y_{i}\left(\xi_{j}\right)=\delta_{i, j}$ for all $i$ and $j$. Also, we set $d_{-}:=|I|$ and we fix a total order in $I$ by numbering its elements, so that $I=\left\{i_{1}, \ldots, i_{d_{-}}\right\}$.

Given $g \in G(A)$, set $\eta_{i}:=g\left(\xi_{i}\right) \in A_{\mathbf{1}}$, for every $i \in I$, and

$$
\gamma_{g}:=\prod_{i=1}^{d_{-}}\left(1+\eta_{i} Y_{i}\right) \in G(A) .
$$

First consider

$$
g_{1}:=g \cdot \gamma_{g}^{-1}=g \cdot \prod_{i=d_{-}}^{1}\left(1+\eta_{i} Y_{i}\right)^{-1}=g \cdot \prod_{i=d_{-}}^{1}\left(1-\eta_{i} Y_{i}\right)
$$

where the product is now in reversed order. Second, as the product in $G(A)=$ $\operatorname{Hom}_{(\operatorname{salg})_{\mathbf{k}}}(\mathcal{O}(G), A)$ is given by convolution, for any $\phi \in\left(\bigwedge W^{H}\right)_{\mathbf{1}}=\mathbb{k}\left[\left\{\xi_{i}\right\}_{i \in I}\right]_{\mathbf{1}}$ we have

$$
\begin{array}{r}
g_{1}(\phi)=\left(g \cdot \prod_{i=d_{-}}^{1}\left(1-\eta_{i} Y_{i}\right)\right)(\phi)=m_{A}\left(\left(g \otimes\left(\bigotimes_{i=d_{-}}^{1}\left(1-\eta_{i} Y_{i}\right)\right)\right)\left(\Delta^{d_{-}}(\phi)\right)\right) \\
=\sum_{(\phi)} g\left(\phi_{(1)}\right) \cdot \prod_{i=d_{-}}^{1}\left(1-\eta_{i} Y_{i}\right)\left(\phi_{(i+1)}\right)
\end{array}
$$

where $\sum_{(\phi)} \phi_{(1)} \otimes \phi_{(2)} \otimes \cdots \otimes \phi_{\left(d_{-}+1\right)}=\Delta^{d_{-}}(\phi)$ as usual. Now, by repeated applications of Lemma 4.3.10 to $H=\mathcal{O}(G)$, we can achieve such an expansion in the form

$$
\Delta^{d_{-}}(\phi)=\sum_{r+s=d_{-}} 1^{\otimes r} \otimes \phi \otimes 1^{\otimes s}+\sum_{(\phi)^{+}} \phi_{(1)} \otimes \phi_{(2)} \otimes \cdots \otimes \phi_{\left(d_{-}+1\right)}
$$


where each monomial $\phi_{(1)} \otimes \cdots \otimes \phi_{\left(d_{-}+1\right)}$ satisfies one of three possible properties, namely:

(I) $\quad \phi_{(1)} \otimes \phi_{(2)} \otimes \cdots \otimes \phi_{\left(d_{-}+1\right)}=1^{\otimes r} \otimes \phi \otimes 1^{\otimes s} \quad$ for any possible $(r, s)$ giving $r+s=d_{-}$— and any such possibility actually occurs;

(II) $\phi_{(\ell)} \in\left(\overline{\mathcal{O}(G)}^{+} \cup \mathcal{O}(G)_{\mathbf{1}}^{[2]}\right)=\left(\mathcal{O}\left(G_{\mathbf{0}}\right)^{+} \cup \mathcal{O}(G)_{\mathbf{1}}^{[2]}\right) \quad$ for some $\quad \ell>1$;

(III) $\phi_{(1)} \otimes \phi_{(2)} \otimes \cdots \otimes \phi_{\left(d_{-}+1\right)} \in \otimes_{s=1}^{d_{-}+1} \mathcal{O}(G)_{\mathbf{1}}^{\left[e_{s}\right]} \quad$ with $\quad \sum_{s=1}^{d_{-}+1} e_{s} \geq 3$.

When case (I) occurs, the contribution to

$$
g_{1}(\phi)=\sum_{(\phi)} g\left(\phi_{(1)}\right) \cdot \prod_{i=d_{-}}^{1}\left(1-\eta_{i} Y_{i}\right)\left(\phi_{(i+1)}\right)
$$

is $g(\phi)$ for $r=0$ and $\left(1-\eta_{\ell} Y_{\ell}\right)(\phi)$ for all $r>1$; then summing over all values of $r \in\left\{0,1, \ldots, d_{-}\right\}$yields the total contribution $g(\phi)+\sum_{r=1}^{d_{-}}\left(1-\eta_{r} Y_{r}\right)(\phi)$.

When case (II) occurs, the product $\prod_{i=d_{-}}^{1}\left(1-\eta_{i} Y_{i}\right)\left(\phi_{(i+1)}\right)$ vanishes; indeed, it contains the zero factor $\left(1-\eta_{\ell} Y_{\ell}\right)\left(\phi_{(\ell+1)}\right)=0$, because we saw above that $\left(1-\eta_{\ell} Y_{\ell}\right)$ vanishes on $\mathcal{O}\left(G_{\mathbf{0}}\right)^{+} \cup \mathcal{O}(G)_{\mathbf{1}}^{[2]}$. So the contribution to $g_{1}(\phi)$ is

$$
g\left(\phi_{(1)}\right) \cdot \prod_{i=d_{-}}^{1}\left(1-\eta_{i} Y_{i}\right)\left(\phi_{(i+1)}\right)=0 .
$$

In the end, when case (III) occurs the product $g\left(\phi_{(1)}\right) \cdot \prod_{i=d_{-}}^{1}\left(1-\eta_{i} Y_{i}\right)\left(\phi_{(i+1)}\right)$ belongs to $\mathfrak{a}_{\mathbf{1}}^{\left[\sum_{s=1}^{d_{-}+1} e_{s}\right]} \subseteq \mathfrak{a}_{\mathbf{1}}^{[3]}$, with $\mathfrak{a}:=\left(\left\{\eta_{i}:=g\left(\xi_{i}\right)\right\}_{i \in I}\right)$ the ideal of $A$ generated by all the $\eta_{i}$ 's. has

Eventually, the outcome is that, for every $\phi \in\left(\bigwedge W^{H}\right)_{\mathbf{1}}=\mathbb{k}\left[\left\{\xi_{i}\right\}_{i \in I}\right]_{\mathbf{1}}$, one

$$
g_{1}(\phi)=g(\phi)+\sum_{\ell=1}^{d_{-}}\left(1-\eta_{\ell} Y_{\ell}\right)(\phi)+\alpha_{3} \quad \text { for some } \quad \alpha_{3} \in \mathfrak{a}_{\mathbf{1}}^{[3]}
$$

We apply this result to $\phi=\xi_{j}$ with $j=1, \ldots, d_{-}$. This yields

$$
\begin{aligned}
g_{1}\left(\xi_{j}\right)=g\left(\xi_{j}\right)+\sum_{\ell=1}^{d_{-}}\left(1-\eta_{\ell} Y_{\ell}\right) & \left(\xi_{j}\right)+\alpha_{3} \\
= & g\left(\xi_{j}\right)+\sum_{\ell=1}^{d_{-}}\left(-\eta_{\ell} \delta_{\ell, j}\right)+\alpha_{3}=\alpha_{3} \in \mathfrak{a}_{1}^{[3]}
\end{aligned}
$$

since $\eta_{j}:=g\left(\xi_{j}\right)$ by construction. Therefore, we have proved the following

Claim. $g_{1}:=g \cdot \gamma_{g}^{-1}$ when restricted to $1 \otimes_{\mathbb{k}} \mathcal{O}\left(G_{\mathbf{1}}\right)=1 \otimes_{\mathbb{k}} \mathbb{k}\left[\left\{\xi_{i}\right\}_{i \in I}\right]$ takes its values in $\mathfrak{a}_{\mathbf{1}}^{(3)}$, the unital $\mathbb{k}$-subalgebra of $A$ generated by $\mathfrak{a}_{1}^{[3]}$.

We can repeat the procedure with $g_{1}$ replacing $g$. Then we consider the corresponding $\gamma_{g_{1}}$, which we use to define $g_{2}:=g_{1} \cdot \gamma_{g_{1}}^{-1}=g \cdot \gamma_{g}^{-1} \cdot \gamma_{g_{1}}^{-1}$. The same arguments - or, more directly, the claim above applied to $g_{1}$ instead of $g$ prove that $g_{2}$ when restricted to $1 \otimes_{\mathbb{k}} \mathcal{O}\left(G_{\mathbf{1}}\right)$ takes its values in $\mathfrak{a}_{\mathbf{1}}^{\left(3^{2}\right)}$, the unital $\mathbb{k}$-subalgebra of $A$ generated by $\mathfrak{a}_{1}^{\left[3^{2}\right]}$.

Iterating the process, we construct elements $g_{s}:=g_{s-1} \cdot \gamma_{g_{s-1}}^{-1}=g \cdot \gamma_{g}^{-1} \cdot \gamma_{g_{1}}^{-1}$. $\gamma_{g_{2}}^{-1} \cdots \gamma_{g_{s-1}}^{-1}$ for increasing $s$ by recursion; their remarkable property is that each $g_{s}$ when restricted to $1 \otimes_{\mathbb{k}} \mathcal{O}\left(G_{\mathbf{1}}\right)$ takes its values in $\mathfrak{a}_{\mathbf{1}}^{\left(3^{s}\right)}$, the unital $\mathbb{k}$-subalgebra of $A$ generated by $\mathfrak{a}_{1}^{\left[3^{s}\right]}$. 
Now, as $\mathfrak{a}$ is an ideal generated by finitely many odd elements, we have $\mathfrak{a}_{1}^{[n]}$ for $n \gg 0$. Thus there exists an $\bar{s} \in \mathbb{N}_{+}$such that $g_{\bar{s}}$ when restricted to $1 \otimes_{\mathbb{k}} \mathcal{O}\left(G_{\mathbf{1}}\right)$ takes its values in $\mathbb{k}$, which means that the restriction of $g_{\bar{s}}$ to $1 \otimes_{\mathbb{k}} \mathcal{O}\left(G_{\mathbf{1}}\right)$ coincides with the counit map of $\mathcal{O}(G)$ followed by the unit map of $A$. But this means that $g_{\bar{s}} \in G_{\mathbf{0}}(A)$, thanks to (4.9).

Finally, from $g_{\bar{s}}=g \cdot \gamma_{g}^{-1} \cdot \gamma_{g_{1}}^{-1} \cdot \gamma_{g_{2}}^{-1} \cdots \gamma_{\bar{g}_{\bar{s}-1}}^{-1}$ we get $g=g_{\bar{s}} \cdot \gamma_{\bar{s}_{\bar{s}-1}} \cdots \gamma_{g_{2}} \cdot \gamma_{g_{1}} \cdot \gamma_{g}$, which shows that $g$ belongs to the subgroup of $G(A)$ generated by $G_{\mathbf{0}}(A)$ and all the $\left(1+\eta_{i} Y_{i}\right)^{\prime}$ 's.

Corollary 4.3.12. Keep notation as in Proposition 4.3.11 above. Fix a total order in $I$, and for any $A \in(\mathrm{salg})_{\mathbb{k}}$ let

$$
G_{\mathbf{1}}(A):=\vec{\prod}_{i \in I}\left(1+A_{\mathbf{1}} Y_{i}\right)=\left\{\vec{\prod}_{i \in I}\left(1+\eta_{i} Y_{i}\right) \mid\left(i, \eta_{i}\right) \in I \times A_{\mathbf{1}}\right\}
$$

where $\vec{\prod}_{i \in I}$ denotes an ordered product. Then there exist group-theoretic factorizations

$$
G(A)=G_{\mathbf{0}}(A) \cdot G_{\mathbf{1}}(A), \quad G(A)=G_{\mathbf{1}}(A) \cdot G_{\mathbf{0}}(A) .
$$

Proof. We apply again, almost verbatim, the proof of Proposition 4.2.7. Indeed, the arguments therein only used the relations mentioned in Lemma 4.2.6, which do hold in $G$.

The previous result can be improved as follows:

Proposition 4.3.13. The factorizations in Corollary 4.3 .12 above correspond to $\mathbb{k}-$ superscheme isomorphisms; namely, the multiplication in $G$ provides $\mathbb{k}$-superscheme isomorphisms

$$
G_{0} \times G_{1} \cong G, \quad G_{1} \times G_{0} \cong G .
$$

Moreover, there exists a $\mathbb{k}$-superscheme isomorphism $\mathbb{A}_{\mathbb{k}}^{0 \mid d_{-}} \cong G_{\mathbf{1}}$ with $d_{-}:=$ $|I|$, given on $A-$ points by

$$
\mathbb{A}_{\mathbb{k}}^{0 \mid d_{-}}(A)=A_{\mathbf{1}}^{d_{-}} \longrightarrow G_{\mathbf{1}}(A), \quad\left(\eta_{i}\right)_{i \in I} \mapsto \prod_{i \in I}^{\rightarrow}\left(1+\eta_{i} Y_{i}\right)
$$

Proof. The statement is a strict analogue of Proposition 4.2.11 and Proposition 4.3.7, and can be proved along the same lines. However, the main technical device — which previously was provided by Lemma 4.2.9 and Lemma 4.3.6 respectively - must now be re-conceived in yet another way, tailored for the present context.

Given $A \in(\mathrm{salg})_{\mathbb{k}}$, assume that one has $\hat{g}_{0} \hat{g}_{1}=\check{g}_{0} \check{g}_{1}$ for some $\hat{g}_{0}, \check{g}_{0} \in G_{\mathbf{0}}(A)$ and $\hat{g}_{1}, \check{g}_{1} \in G_{\mathbf{1}}(A)$; we number the elements of $I$ following their order, so that we can write

$$
\hat{g}_{1}=\prod_{i \in I}^{\rightarrow}\left(1+\hat{\eta}_{i} Y_{i}\right)=\prod_{i=1}^{d_{-}}\left(1+\hat{\eta}_{i} Y_{i}\right), \quad \check{g}_{1}=\prod_{i \in I}^{\rightarrow}\left(1+\check{\eta}_{i} Y_{i}\right)=\prod_{i=1}^{d_{-}}\left(1+\check{\eta}_{i} Y_{i}\right)
$$

for some $\hat{\eta}_{i}, \check{\eta}_{i} \in A_{\mathbf{1}}$.

We shall prove now that $\hat{\eta}_{i}=\check{\eta}_{i}$ for all $i \in I$. In particular, assuming $\hat{g}_{0}=\check{g}_{0}$ this is enough to prove the last part of the statement; namely, this proves the injectivity of the superscheme morphism therein, whose surjectivity is automatic. In addition, this also implies that $\hat{g}_{1}=\check{g}_{1}$, whence (as $\hat{g}_{0} \hat{g}_{1}=\check{g}_{0} \check{g}_{1}$ by assumption) it follows $\hat{g}_{0}=\check{g}_{0}$ too. 
Letting $H:=\mathcal{O}(G)$, we act much like in the proof of Proposition 4.3.11. Therefore, for any $\phi \in\left(\bigwedge W^{H}\right)_{1}=\mathcal{O}\left(G_{1}\right)_{1}$ we find

$$
\left(\hat{g}_{0} \hat{g}_{1}\right)(\phi)=\hat{g}_{0}\left(\phi_{(1)}\right) \hat{g}_{1}\left(\phi_{(2)}\right)=\hat{g}_{0}(\phi)+\hat{g}_{1}(\phi)+\hat{g}_{0}\left(\phi_{(1)}^{+}\right) \hat{g}_{1}\left(\phi_{(2)}^{+}\right)=\hat{g}_{1}(\phi)
$$

because $\Delta(\phi)=\phi_{(1)}^{+} \otimes \phi_{(2)}^{+} \equiv 1 \otimes \phi \bmod \left(J_{H} \otimes_{\mathbb{k}} H\right)$, by the fact that $H=\mathcal{O}(G)$ is strongly split (see Theorem 3.2.8), and $g_{0}\left(J_{H}\right)=\{0\}$. The same occurs of course for " $\breve{g}$ " replacing " $\hat{g}$ " everywhere; hence in the end we have

$$
\left(\hat{g}_{0} \hat{g}_{1}\right)(\phi)=\hat{g}_{1}(\phi), \quad\left(\check{g}_{0} \check{g}_{1}\right)(\phi)=\check{g}_{1}(\phi) \quad \forall \phi \in \mathcal{O}\left(G_{\mathbf{1}}\right)_{\mathbf{1}} .
$$

On the other hand we have

$$
\hat{g}_{1}(\phi)=\left(\prod_{i=1}^{d_{-}}\left(1+\hat{\eta}_{i} Y_{i}\right)\right)(\phi)=\sum_{(\phi)} \prod_{i=1}^{d_{-}}\left(1+\hat{\eta}_{i} Y_{i}\right)\left(\phi_{(i)}\right)
$$

where $\sum_{(\phi)} \phi_{(1)} \otimes \phi_{(2)} \otimes \cdots \otimes \phi_{\left(d_{-}\right)}=\Delta^{d_{--1}(\phi)}$ as usual. Now, like for Proposition 4.3.11, by repeatedly applying Lemma 4.3.10 we eventually find, for every $\phi \in$ $\left(\bigwedge W^{H}\right)_{\mathbf{1}}=\mathbb{k}\left[\left\{\xi_{i}\right\}_{i \in I}\right]_{\mathbf{1}}$,

$$
\hat{g}_{1}(\phi)=\sum_{\ell=1}^{d_{-}}\left(1-\eta_{\ell} Y_{\ell}\right)(\phi)+\alpha_{\phi}(\underline{\hat{\eta}})
$$

where $\alpha_{\phi}=\alpha_{\phi}(\underline{\eta})=\alpha_{\phi}\left(\eta_{1}, \ldots, \eta_{d_{-}}\right)$is some polynomial (depending on $\phi$ ) in the variables $\eta_{1}, \ldots, \eta_{d_{-}} \in A_{1}$ in which only monomials may occur whose degree is odd and at least three. Applying all this $\phi=\xi_{j}\left(j=1, \ldots, d_{-}\right)$and writing $\alpha_{j}:=\alpha_{\xi_{j}}$ for each $j$, we get

$$
\hat{g}_{1}\left(\xi_{j}\right)=\sum_{\ell=1}^{d_{-}}\left(1-\hat{\eta}_{\ell} Y_{\ell}\right)\left(\xi_{j}\right)+\alpha_{j}(\underline{\hat{\eta}})=\sum_{\ell=1}^{d_{-}} \hat{\eta}_{\ell} \delta_{\ell, j}+\alpha_{j}(\underline{\hat{\eta}})=\hat{\eta}_{j}+\alpha_{j}(\underline{\hat{\eta}}) .
$$

Clearly, the parallel result holds for $\check{g}_{1}$; hence in the end we have (with the same $\alpha_{j}$ twice!)

$$
\hat{g}_{1}\left(\xi_{j}\right)=\hat{\eta}_{j}+\alpha_{j}(\underline{\hat{\eta}}), \quad \check{g}_{1}\left(\xi_{j}\right)=\check{\eta}_{j}+\alpha_{j}(\underline{\check{\eta}}) \quad \forall j \in I .
$$

As $\hat{g}_{0} \hat{g}_{1}=\check{g}_{0} \check{g}_{1}$, from (4.10) and (4.11) we get $\hat{\eta}_{j}+\alpha_{j}(\underline{\hat{\eta}})=\check{\eta}_{j}+\alpha_{j}(\underline{\check{\eta}})$ for all $j \in I$. As a last remark, we notice that $\left(\eta_{j} \mapsto \eta_{j}+\alpha_{j}(\underline{\eta}), \forall j \in I\right)$ defines (the value on $A$-points of) a $\mathbb{k}$-superscheme automorphism of $\mathbb{A}_{\mathbb{k}}^{0 \mid d_{-}}$. Therefore, from $\hat{\eta}_{j}+\alpha_{j}(\underline{\hat{\eta}})=\check{\eta}_{j}+\alpha_{j}(\underline{\check{\eta}})$ for all $j \in I$ we eventually get $\hat{\eta}_{j}=\check{\eta}_{j}$ for all $j \in I$.

We are ready for the next result, the main one of the present section, which extends Theorem 4.2.14

Theorem 4.3.14. The functor $\Psi_{g}:(\mathrm{sHCp})_{\mathbb{k}} \longrightarrow$ (gss-fsgroups $)_{\mathbb{k}}$ is inverse, up to a natural isomorphism, to the functor $\Phi_{g}$ : (gss-fsgroups) $)_{\mathbb{k}} \longrightarrow(\mathrm{sHCp})_{\mathbb{k}}$. In other words, the two of them are category equivalences, quasi-inverse to each other.

Proof. In the proof of Proposition 4.3.9 we saw that, for $\mathcal{P}=\left(G_{+}, \mathfrak{g}\right) \in(\mathrm{sHCp})_{\mathbb{k}_{\mathrm{k}}}$ and $G_{\mathcal{P}}:=\Psi_{g}(\mathcal{P})$, we have $\left(G_{\mathcal{P}}\right)_{0}=G_{+}$and $\operatorname{Lie}\left(G_{\mathcal{P}}\right)=\mathfrak{g}$, thus $\Phi_{g}\left(\Psi_{g}(\mathcal{P})\right)=\mathcal{P}$. So in one direction we are done.

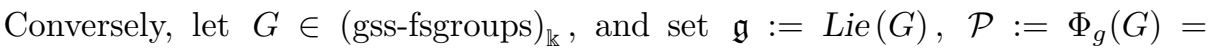
$\left(G_{\mathbf{0}}, \mathfrak{g}\right)$. We look at the supergroup $\Psi_{g}\left(\Phi_{g}(G)\right)=\Psi_{g}(\mathcal{P}):=G_{\mathcal{P}}$, aiming to prove that it is naturally isomorphic to $G$. 
Given $A \in(\mathrm{salg})_{\mathbb{k}}$, by abuse of notation we denote with the same symbols any element $g_{0} \in G_{\mathbf{0}}(A)$ as belonging to $G(A)$ — via the embedding of $G_{\mathbf{0}}(A)$ into $G(A)$ - and as an element of $G_{\mathcal{P}}(A)$ - actually, one of the distinguished generators given from scratch.

With this convention, it is immediate to see that Lemma 4.2.6 yields the following: there exists a unique group morphism $\Omega_{A}: G_{\mathcal{P}}(A) \longrightarrow G(A)$ such that $\Omega_{A}\left(g_{0}\right)=g_{0}$ for all $g_{0} \in G_{\mathbf{0}}(A)$ and $\Omega_{A}\left(\left(1+\eta_{i} Y_{i}\right)\right)=\left(1+\eta_{i} Y_{i}\right)$ for all $\eta_{i} \in A_{\mathbf{1}}$, $i \in I$.

By Proposition 4.3.11 above we have that the morphism $\Omega_{A}$ is actually surjective. On the other hand, the direct product factorizations for $G_{\mathcal{P}}$ (see Proposition 4.3.7) and for $G$ (see Proposition 4.3.13) easily imply that the morphism $\Omega_{A}$ is also injective, hence it is a group isomorphism. Finally, it is clear that the morphisms $\Omega_{A}$ 's are natural in $A$, thus overall they provide an isomorphism between $G_{\mathcal{P}}=\Psi_{g}\left(\Phi_{g}(G)\right)$ and $G$, which ends the proof.

4.3.15. An alternative realization of $\mathbf{G}_{\mathcal{P}}$. Let $\mathcal{P}=\left(G_{+}, \mathfrak{g}\right) \in(\mathrm{sHCp})_{\mathbb{k}}$ be a super Harish-Chandra pair; we now present a different way of realizing the $\mathbb{k}$-supergroup $G_{\mathcal{P}}$ introduced in Definition 4.3.2 (a). In the following, if $K$ is any group presented by generators and relations, we write $K=\langle\Gamma\rangle /(\mathcal{R})$ if $\Gamma$ is a set of free generators (of $K$ ), $\mathcal{R}$ is a set of "relations" among generators and $(\mathcal{R}$ ) is the normal subgroup in $K$ generated by $\mathcal{R}$. As a matter of notation, given a presentation $K=\langle\Gamma\rangle /(\mathcal{R})=\langle\Gamma\rangle /\left(\mathcal{R}_{1} \cup \mathcal{R}_{2}\right)$ with $\mathcal{R}=\mathcal{R}_{1} \cup \mathcal{R}_{2}$, the Double Quotient Theorem gives us

$$
\begin{aligned}
K=\langle\Gamma\rangle /(\mathcal{R}) & =\langle\Gamma\rangle /\left(\mathcal{R}_{1} \cup \mathcal{R}_{2}\right) \\
& =\langle\Gamma\rangle /\left(\mathcal{R}_{1}\right) /\left(\mathcal{R}_{1} \cup \mathcal{R}_{2}\right) /\left(\mathcal{R}_{1}\right)=\langle\bar{\Gamma}\rangle /\left(\overline{\mathcal{R}_{2}}\right)
\end{aligned}
$$

where $\bar{\Gamma}$ and $\overline{\mathcal{R}_{2}}$ respectively denote the images of $\Gamma$ and of $\mathcal{R}_{2}$ in the quotient $\operatorname{group}\langle\Gamma\rangle /\left(\mathcal{R}_{1}\right)$.

For a fixed $A \in(\operatorname{salg})_{\mathbb{k}}$, we consider $G_{+}(A)$ and inside it the normal subgroup $G \approx(A)$ given by

$$
G \approx(A):=\left\langle\left\{g_{+} \in G_{+}(A) \mid g_{+}=\left(1+\eta^{\prime} \eta^{\prime \prime} X\right), \eta^{\prime}, \eta^{\prime \prime} \in A_{\mathbf{1}}, X \in\left[\mathfrak{g}_{\mathbf{1}}, \mathfrak{g}_{\mathbf{1}}\right] \cup \mathfrak{g}_{\mathbf{1}}^{\langle 2\rangle}\right\}\right\rangle .
$$

Then consider also the three sets

$$
\Gamma_{A}^{+}:=G_{+}(A), \quad \Gamma_{A}^{\approx}:=G_{\approx}(A), \quad \Gamma_{A}^{-}:=\Gamma_{A} \cup\{(1+\eta Y)\}_{(Y, \eta) \in \mathfrak{g}_{1} \times A_{1}}
$$

and the sets of relations (for $g_{+}, g_{+}^{\prime}, g_{+}^{\prime \prime} \in G_{+}(A), g_{\approx},, g_{\approx}^{\prime \prime} \in G_{\approx}(A), \eta, \eta^{\prime}, \eta^{\prime \prime} \in$ $A_{1}, X \in\left[\mathfrak{g}_{1}, \mathfrak{g}_{1}\right] \cup \mathfrak{g}_{1}^{\langle 2\rangle}, Y, Y^{\prime}, Y^{\prime \prime} \in \mathfrak{g}_{\mathbf{1}}$, with $\dot{G}_{+}$and $\dot{G}_{\approx}$ being the product in 
$G_{+}(A)$ and in $G_{\approx}(A)$ respectively)

$$
\begin{aligned}
& \mathcal{R}_{A}^{+}: \quad g_{+}^{\prime} \cdot g_{+}^{\prime \prime}=g_{+\dot{G}_{+}}^{\prime} g_{+}^{\prime \prime} \\
& \left\{\begin{array}{c}
g_{\approx}^{\prime} \cdot g_{\approx}^{\prime \prime}=g_{\approx \dot{\sigma}}^{\prime} \approx g_{\approx}^{\prime \prime} \\
(1+\eta Y) \cdot g_{\approx}=g_{\approx} \cdot(1+\eta Y) \cdot\left(1+\eta \operatorname{Ad}\left(g_{\approx}^{-1}\right)(Y)\right)
\end{array}\right. \\
& \mathcal{R}_{A}^{-}:\left\{\begin{array}{c}
\left(1+\eta^{\prime} Y\right) \cdot\left(1+\eta^{\prime \prime} Y\right)=\left(1+\eta^{\prime \prime} \eta^{\prime} Y^{\langle 2\rangle}\right) \cdot\left(1+\left(\eta^{\prime}+\eta^{\prime \prime}\right) Y\right) \\
\left(1+\eta^{\prime \prime} Y^{\prime \prime}\right) \cdot\left(1+\eta^{\prime} Y^{\prime}\right)=\left(1+\eta^{\prime} \eta^{\prime \prime}\left[Y^{\prime}, Y^{\prime \prime}\right]\right) \cdot\left(1+\eta^{\prime} Y^{\prime}\right) \cdot\left(1+\eta^{\prime \prime} Y^{\prime \prime}\right) \\
\left(1+\eta Y^{\prime}\right) \cdot\left(1+\eta Y^{\prime \prime}\right)=\left(1+\eta\left(Y^{\prime}+Y^{\prime \prime}\right)\right)
\end{array}\right. \\
& \left(1+\eta 0_{\mathfrak{g}_{1}}\right)=1, \quad\left(1+0_{A} Y\right)=1, \\
& \mathcal{R}_{A}^{\ltimes}: \quad g_{\approx} \cdot g_{+}=g_{+} \cdot\left(g_{+}^{-1} \dot{G}_{+} g_{\approx_{\dot{G}_{+}}} g_{+}\right), \quad(1+\eta Y) \cdot g_{+}=g_{+} \cdot\left(1+\eta \operatorname{Ad}\left(g_{+}^{-1}\right)(Y)\right) \\
& \mathcal{R} \approx \tilde{A}^{\prime}: \quad\left(g_{\approx}\right)_{\Gamma_{A}^{+}}=\left(g_{\approx}\right)_{\Gamma_{\tilde{A}}} \\
& \mathcal{R}_{A}:=\mathcal{R}_{A}^{+} \cup \mathcal{R}_{A}^{-} \cup \mathcal{R}_{A}^{\ltimes} \cup \mathcal{R}_{A} \tilde{A}
\end{aligned}
$$

and define a new group, by generators and relations, as $G_{-}(A):=\left\langle\Gamma_{A}^{-}\right\rangle /\left(\mathcal{R}_{A}^{-}\right)$.

It follows from Remarks 4.3.3 (c) that

$$
G_{\mathcal{P}}(A)=\left\langle\Gamma_{A}^{+} \cup \Gamma_{A}^{-}\right\rangle /\left(\mathcal{R}_{A}\right)=\left\langle\Gamma_{A}^{+} \cup \Gamma_{A}^{-}\right\rangle /\left(\mathcal{R}_{A}^{+} \cup \mathcal{R}_{A}^{-} \cup \mathcal{R}_{A}^{\ltimes} \cup \mathcal{R}_{\tilde{A}}\right) .
$$

Indeed, we are just taking larger sets of generators and of relations, with enough redundancies as to get in the end a different presentation of the same group.

From this we find a neat description of $G_{\mathcal{P}}(A)$ by achieving the presentation (4.12) in a series of intermediate steps; namely adding only one bunch of relations at a time. As a first step, we have

$$
\begin{aligned}
& \left\langle\Gamma_{A}^{+} \cup \Gamma_{A}^{-}\right\rangle /\left(\mathcal{R}_{A}^{+} \cup \mathcal{R}_{A}^{-}\right) \\
& \quad=\left\langle\Gamma_{A}^{+}\right\rangle /\left(\mathcal{R}_{A}^{+}\right) *\left\langle\Gamma_{A}^{-}\right\rangle /\left(\mathcal{R}_{A}^{-}\right) \cong G_{+}(A) * G_{-}(A)
\end{aligned}
$$

where $G_{+}(A) \cong\left\langle\Gamma_{A}^{+}\right\rangle /\left(\mathcal{R}_{A}^{+}\right)$by construction and $*$ denotes the free product (of two groups).

For the next two steps we can follow two different lines of action. On the one hand, one has

$$
\begin{aligned}
\left\langle\Gamma_{A}^{+} \cup \Gamma_{A}^{-}\right\rangle /\left(\mathcal{R}_{A}^{+} \cup \mathcal{R}_{A}^{-}\right. & \left.\cup \mathcal{R}_{A}^{\ltimes}\right) \\
& \cong\left(G_{+}(A) * G_{-}(A)\right) /\left(\overline{\mathcal{R}_{A}^{\ltimes}}\right) \cong G_{+}(A) \ltimes G_{-}(A)
\end{aligned}
$$

because of (4.12) and (4.14) together, where $G_{+}(A) \ltimes G_{-}(A)$ is the semidirect product of $G_{+}(A)$ with $G_{-}(A)$ with respect to the obvious ("adjoint") action of the former on the latter. Then

$$
\begin{aligned}
& \left\langle\Gamma_{A}^{+} \cup \Gamma_{A}^{-}\right\rangle /\left(\mathcal{R}_{A}\right) \cong\left\langle\Gamma_{A}^{+} \cup \Gamma_{A}^{-}\right\rangle /\left(\mathcal{R}_{A}^{+} \cup \mathcal{R}_{A}^{-} \cup \mathcal{R}_{A}^{\ltimes} \cup \mathcal{R}_{A} \widetilde{A}\right) \\
& \cong\left(G_{+}(A) \ltimes G_{-}(A)\right) /\left(\overline{\mathcal{R}_{\tilde{A}}}\right) \cong\left(G_{+}(A) \ltimes G_{-}(A)\right) / N_{\approx}(A)
\end{aligned}
$$


where $N_{\approx}(A)$ is the normal subgroup of $G_{+}(A) \ltimes G_{-}(A)$ generated by

$$
\left\{\left(g_{\approx}, g_{\approx}^{-1}\right) \mid g_{\approx} \in G \approx(A)\right\} .
$$

This together with (4.13) eventually yields

$$
G_{\mathcal{P}}(A)=\left(G_{+}(A) \ltimes G_{-}(A)\right) / N_{\approx}(A) .
$$

On the other hand, again from (4.12) and (4.14) together we get

$$
\begin{aligned}
\left\langle\Gamma_{A}^{+} \cup \Gamma_{A}^{-}\right\rangle /\left(\mathcal{R}_{A}^{+} \cup \mathcal{R}_{A}^{-}\right. & \left.\cup \mathcal{R}_{\tilde{A}}^{\approx}\right) \\
& \cong G_{+}(A) * G_{-}(A) /\left(\overline{\mathcal{R}_{\tilde{A}}}\right) \cong G_{+}(A)_{G \approx(A)}^{*} G_{-}(A)
\end{aligned}
$$

where $G_{+}(A) \underset{G \approx(A)}{*} G_{-}(A)$ is the amalgamated product of $G_{+}(A)$ and $G_{-}(A)$ over $G_{\approx}(A)$ with respect to the obvious natural monomorphisms $G_{\approx}(A) \longrightarrow G_{+}(A)$ and $G_{\approx}(A) \longrightarrow G_{-}(A)$. Then

$$
\begin{aligned}
& \left\langle\Gamma_{A}^{+} \cup \Gamma_{A}^{-}\right\rangle /\left(\mathcal{R}_{A}\right) \cong\left\langle\Gamma_{A}^{+} \cup \Gamma_{A}^{-}\right\rangle /\left(\mathcal{R}_{A}^{+} \cup \mathcal{R}_{A}^{-} \cup \mathcal{R}_{A}^{\approx} \cup \mathcal{R}_{A}^{\ltimes}\right) \\
& \cong\left(G_{+}(A)_{G_{\approx}(A)}^{*} G_{-}(A)\right) /\left(\overline{\mathcal{R}_{A}^{\ltimes}}\right) \cong\left(G_{+}(A) \underset{G_{\approx}(A)}{*} G_{-}(A)\right) / N_{\ltimes}(A)
\end{aligned}
$$

where $N_{\ltimes}(A)$ is the normal subgroup of $G_{+}(A) \underset{G \approx(A)}{*} G_{-}(A)$ generated by

$$
\begin{aligned}
\left\{g_{+} \cdot(1+\eta Y)\right. & \left.\cdot g_{+}^{-1} \cdot\left(1+\eta \operatorname{Ad}\left(g_{+}\right)(Y)\right)^{-1}\right\}_{(Y, \eta) \in \mathfrak{g}_{1} \times A_{1}, g_{+} \in G_{+}(A)} \\
& \cup\left\{g_{+} \cdot g_{\approx} \cdot g_{+} \cdot\left(g_{+\dot{G}_{+}} g_{\approx} \dot{G}_{+} g_{+}\right)^{-1}\right\}_{g_{+} \in G_{+}(A), g_{\approx} \in G_{\approx}(A)}
\end{aligned}
$$

All this along with (4.13) eventually gives

$$
G_{\mathcal{P}}(A)=\left(G_{+}(A) \underset{G_{\approx}(A)}{*} G_{-}(A)\right) / N_{\ltimes}(A)
$$

for all $A \in(\mathrm{salg})_{\mathbb{k}}$. In functorial terms this yields

$$
\begin{gathered}
G_{\mathcal{P}}=\left(G_{+} \ltimes G_{-}\right) / N_{\approx} \quad \text { and } \quad G_{\mathcal{P}}=\left(G_{+} * G_{G^{\approx}}^{*}\right) / N_{\ltimes} \\
\text { or } \quad G_{\mathcal{P}}=\left(G_{+} \underset{G \approx}{\ltimes} G_{-}\right)
\end{gathered}
$$

where the last, (hopefully) more suggestive notation $G_{\mathcal{P}}=\left(G_{+} \underset{G_{\approx}}{\ltimes} G_{-}\right)$tells us that $G_{\mathcal{P}}$ is the "amalgamated semidirect product" of $G_{+}$and $G_{-}$over their common subgroup $G \approx$.

4.4. Examples, applications, generalizations. We shall now illustrate how the equivalence we established between (globally strongly split fine) affine supergroups and super Harish-Chandra pairs applies to specific examples. In particular, we show that one recovers the construction of "Chevalley supergroups" as presented in 10 13]. 
We also have applications to representation theory. First, if $G$ and $\left(G_{+}, \mathfrak{g}\right)$ respectively are a supergroup and an $\mathrm{sHCp}$ which correspond to each other under the previously mentioned equivalence, then we shall find an equivalence between the category of (left or right) $G$-modules and the category of $\left(G_{+}, \mathfrak{g}\right)$-modules. Second, given a supergroup $G \in$ (gss-fsgroups) $)_{\mathbb{k}}$ and any $G_{\mathbf{0}}$-module $V$ we provide an explicit construction of the induced $G$-module $\operatorname{Ind}_{G_{0}}^{G}(V)$.

Finally, we discuss the possibility of extending our results to a more general setup.

4.4.1. The example of "Chevalley supergroups". Let $\mathfrak{g}$ be a simple Lie superalgebra over an algebraically closed field $\mathbb{K}$ of characteristic zero. A complete classification of these objects was found by Kac (and others; see e.g. [14]), who split them in two main (disjoint) families: those of "classical" type - still divided into "basic" and "strange" types - and those of "Cartan" type.

In a series of papers, Fioresi and Gavarini devised a systematic procedure to find affine $\mathbb{Z}$-supergroups $G$ having the given $\mathfrak{g}$ as tangent Lie superalgebra - see $10-12$ for the classical type, and [13] for the Cartan type. Indeed, the outcome there is an explicit recipe to construct all supergroups of this type which in addition are connected. Their construction starts with a faithful, finite-dimensional $\mathfrak{g}$-module $V$, and eventually realizes one model of the required $\mathbb{Z}$-supergroup $G$ as a closed $\mathbb{Z}$-subsupergroup of GL( $V)$. The procedure mimics and extends the classical one developed by Chevalley to construct (connected) algebraic groups associated with any simple Lie algebra over $\mathbb{K}$. For this reason, the resulting supergroups are named "Chevalley supergroups".

On the other hand, if one revisits the work of Fioresi and Gavarini in the spirit of the present paper, one realizes the following: the construction of Fioresi and Gavarini is nothing but a special - and peculiar, for extra features occur, of course - instance of Theorem 4.2.14. Indeed, once $\mathfrak{g}$, a faithful $\mathfrak{g}$-module $V$, and suitable $\mathbb{Z}$-forms $\mathfrak{g}_{\mathbb{Z}}$ and $V_{\mathbb{Z}}$ of them are fixed, one can consider the even part $\mathfrak{g}_{\mathbf{0}}$ and realize, following Chevalley, a (classical) affine group-scheme $G_{\mathbf{0}}$ over $\mathbb{Z}$ which is a (connected) closed subgroup-scheme of $\operatorname{GL}(V)$ such that Lie $\left(G_{\mathbf{0}}\right)=\mathfrak{g}_{\mathbf{0}}$. Then $\mathcal{P}:=$ $\left(\left(G_{\mathbf{0}}, \mathfrak{g}\right), V\right)$ is a linear super Harish-Chandra pair over $\mathbb{Z}$, i.e., $\mathcal{P} \in(\mathrm{lsHCp})_{\mathbb{Z}}$.

Now, after Theorem 4.2.14 it makes sense to consider the associated linear fine supergroup $G_{\mathcal{P}}:=\Psi_{\ell}(\mathcal{P}) \in(\text { lgss-fsgroups })_{\mathbb{Z}}$ over $\mathbb{Z}$. Then a direct comparison shows that the very definition of this $G_{\mathcal{P}}$ actually coincides with the definition of the $\mathbb{Z}$-supergroup $G_{V}$ provided by the recipe of Fioresi and Gavarini. Indeed, we can say that Fioresi and Gavarini's construction consists in "composing" Chevalley's classical construction - to produce a group scheme $G_{+}$out of a faithful $\mathfrak{g}_{+}$-module $V_{+}$, if $\mathfrak{g}_{+}$is a Lie algebra (plus technicalities) — and (as a second step) the functor $\Psi_{\ell}$. It follows then that all "Chevalley supergroups" (both of classical or of Cartan type) as provided by Fioresi and Gavarini are linear fine supergroups; in particular, they are globally strongly split.

4.4.2. Representations 1: The equivalence [supergroup modules $\simeq$ sHCp-modules]. An important byproduct of the equivalence between (gss-fsgroups) $)_{\mathbb{k}}$ and $(\mathrm{sHCp})_{\mathbb{k}_{\mathrm{k}}}$ comes as an application to representation theory. Indeed, let $G \in$ (gss-fsgroups $)_{\mathbb{k}}$ and $\mathcal{P} \in(\mathrm{sHCp})_{\mathbb{k}}$ respectively be a supergroup and an $\mathrm{sHCp}$ which correspond to each other through the above-mentioned equivalence - namely, $G=\Psi_{g}(\mathcal{P})$ 
and $\mathcal{P}=\Phi_{g}(G)$. Then we let $G$-Mod and $\mathcal{P}$-Mod respectively be the category of $G$-supermodules and of $\mathcal{P}$-modules; in short, here we mean that a $G$-module is the datum of a finite free $\mathbb{k}$-supermodule $M^{\prime}$ with a morphism $\Omega: G \longrightarrow \operatorname{GL}\left(M^{\prime}\right)$ of supergroups, i.e., in (sgroups) $)_{\mathbb{k}}$, whereas a $\mathcal{P}$-module is the datum of a finite free $\mathbb{k}$-supermodule $M^{\prime \prime}$ with a morphism $\left(\Omega_{+}, \omega\right): \mathcal{P} \longrightarrow\left(\operatorname{GL}\left(M^{\prime \prime}\right)_{0}, \mathfrak{g l}\left(M^{\prime \prime}\right)\right)$ of sHCp's, i.e., in $(\mathrm{sHCp})_{\mathbb{k}}$.

Now assume $M^{\prime}$ is a $G$-module. Applying $\Phi_{g}:(\text { gss-fsgroups })_{\mathbb{k}} \longrightarrow(\mathrm{sHCp})_{\mathbb{k}}$ to the morphism $\Omega: G \longrightarrow \mathrm{GL}\left(M^{\prime}\right)$ we find a morphism $\Phi_{g}(\Omega): \Phi_{g}(G) \longrightarrow$ $\Phi_{g}\left(\mathrm{GL}\left(M^{\prime}\right)\right)$ between the corresponding objects in $(\mathrm{sHCp})_{\mathbb{k}}$. But $\Phi_{g}(G)=\mathcal{P}$ by assumption and $\Phi_{g}\left(\mathrm{GL}\left(M^{\prime}\right)\right)=\left(\mathrm{GL}\left(M^{\prime}\right)_{\mathbf{0}}, \mathfrak{g l}\left(M^{\prime}\right)\right)$, so what we have is a morphism $\Phi_{g}(\Omega): \mathcal{P} \longrightarrow\left(\mathrm{GL}\left(M^{\prime}\right)_{\mathbf{0}}, \mathfrak{g l}\left(M^{\prime}\right)\right)$ making $M^{\prime}$ into a $\mathcal{P}$-module.

Conversely, let $M^{\prime \prime}$ be a $\mathcal{P}$-module. Applying the functor $\Psi_{g}:(\mathrm{sHCp})_{\mathbb{k}} \longrightarrow$ (gss-fsgroups $)_{\mathbb{k}}$ to the corresponding morphism $\left(\Omega_{+}, \omega\right): \mathcal{P} \rightarrow\left(\operatorname{GL}\left(M^{\prime \prime}\right)_{\mathbf{0}}, \mathfrak{g l}\left(M^{\prime \prime}\right)\right)$ we get a morphism $\Psi_{g}\left(\left(\Omega_{+}, \omega\right)\right): \Psi_{g}(\mathcal{P}) \longrightarrow \Psi_{g}\left(\left(\operatorname{GL}\left(M^{\prime \prime}\right)_{\mathbf{0}}, \mathfrak{g l}\left(M^{\prime \prime}\right)\right)\right)$ between the corresponding supergroups. As $\Psi_{g}(\mathcal{P})=G$ by our assumptions while $\Psi_{g}\left(\left(\operatorname{GL}\left(M^{\prime \prime}\right)_{\mathbf{0}}, \mathfrak{g l}\left(M^{\prime \prime}\right)\right)\right)=\mathrm{GL}\left(M^{\prime \prime}\right)$, we find a morphism $\Psi_{g}\left(\left(\Omega_{+}, \omega\right)\right): G \longrightarrow$ $\mathrm{GL}\left(M^{\prime \prime}\right)$ in (gss-fsgroups) $)_{\mathbb{k}}$ which makes $M^{\prime \prime}$ into a $G$-module.

The reader can easily check that the previous discussion has the following outcome:

Theorem 4.4.3. Let $G \in(\text { gss-fsgroups })_{\mathbb{k}}$ and $\mathcal{P} \in(\mathrm{sHCp})_{\mathbb{k}}$ correspond to each other as above.

(a) For any fixed finite free $\mathbb{k}$-supermodule $M$, the above constructions provide two bijections, inverse to each other, between $G$-module structures and $\mathcal{P}$-module structures on $M$.

(b) The whole construction above is natural in $M$, in that the above bijections over two $\mathbb{k}$-supermodules $\widehat{M}$ and $\widetilde{M}$ are compatible with $\mathbb{k}$-supermodule morphisms from $\widehat{M}$ to $\widetilde{M}$. Thus, all the bijections mentioned in (a) - for all different $M$ 's - do provide equivalences, quasi-inverse to each other, between the category of all $G-$ modules and the category of all $\mathcal{P}$-modules.

Remarks 4.4.4. Professor Masuoka kindly shared with the author the following observations:

(a) Here above we considered modules $M$ (over supergroups or over sHCp's) that are finite free as $\mathbb{k}$-supermodules - which is consistent with our description of $\operatorname{GL}(M)$ as given in Examples 2.1.5(b). On the other hand, one can weaken this assumption, requiring only that $M$ be finite projective; Theorem 4.4 .3 above will then still hold true.

(b) With the above-mentioned projectivity assumption, our Theorem $4.4 .3 \mathrm{im}$ proves Proposition 5.4 and Theorem 5.8 in 20. Indeed, Proposition 5.4 holds true over any commutative ring, just assuming (with notation of 20] ) that the map $O(G) \longrightarrow$ hy $(G)^{*}$ be injective (geometrically, it means that $G$ is connected).

4.4.5. Representations 2: Induction from $G_{\mathbf{0}}$ to $G$. Let a supergroup $G \in$ (gss-fsgroups) $)_{\mathbb{k}}$ be given, with associated classical subsupergroup $G_{e v}=G_{\mathbf{0}}$. Let $V$ be any $G_{0^{-}}$-module. We shall now provide an explicit construction of the induced $G-$ module $\operatorname{Ind}_{G_{0}}^{G}(V)$. 
Being a $G_{\mathbf{0}^{-}}$-module, $V$ is also, automatically, a $\mathfrak{g}_{\mathbf{0}}-$ module. Then one does have the induced $\mathfrak{g}$-module $\operatorname{Ind}_{\mathfrak{g}_{0}}^{\mathfrak{g}}(V)$, which can be realized as

$$
\operatorname{Ind}_{\mathfrak{g}_{0}}^{\mathfrak{g}}(V)=\operatorname{Ind}_{U\left(\mathfrak{g}_{\mathbf{0}}\right)}^{U(\mathfrak{g})}(V)=U(\mathfrak{g}) \otimes_{U\left(\mathfrak{g}_{\mathbf{0}}\right)} V .
$$

By construction, it is clear that this bears also a unique structure of $G_{0^{-}}$-module which is compatible with the $\mathfrak{g}$-action and coincides with the original $G_{0^{-}}$action on $\mathbb{k} \otimes_{U\left(\mathfrak{g}_{\mathbf{0}}\right)} V \cong V$ given from scratch. Indeed, we can describe explicitly this $G_{0^{-}}$action, as follows. First, by construction we have

$$
\operatorname{Ind}_{\mathfrak{g}_{0}}^{\mathfrak{g}}(V)=U(\mathfrak{g}) \otimes_{U\left(\mathfrak{g}_{0}\right)} V=\bigwedge \mathfrak{g}_{1} \otimes_{\mathbb{k}} V
$$

- because $U(\mathfrak{g}) \cong \bigwedge \mathfrak{g}_{1} \otimes_{\mathbb{k}} U\left(\mathfrak{g}_{\mathbf{0}}\right)$ as a $\mathbb{k}$-module, by the PBW theorem for Lie superalgebras; see $(4.7)$ - with the $\mathfrak{g}_{0}$-action given by $x \cdot(y \otimes v)=\operatorname{ad}(x)(y) \otimes$ $v+y \otimes(x . v)$ for $x \in \mathfrak{g}_{\mathbf{0}}, y \in \bigwedge \mathfrak{g}_{1}, v \in V$, where by ad we denote the unique $\mathfrak{g}_{0}$-action on $\Lambda \mathfrak{g}_{1}$ by algebra derivations induced by the adjoint $\mathfrak{g}_{0}$-action on $\mathfrak{g}_{1}$. Second, this action clearly integrates to a (unique) $G_{0^{-}}$action given by $g_{0} \cdot(y \otimes v):=$ $A d\left(g_{0}\right)(y) \otimes\left(g_{0} \cdot v\right)$ for $g_{0} \in G_{\mathbf{0}}, y \in \Lambda \mathfrak{g}_{\mathbf{1}}, v \in V$, where we write Ad for the unique $G_{0^{-}}$action on $\bigwedge \mathfrak{g}_{1}$ by algebra automorphisms induced by the adjoint $G_{0^{-}}$ action on $\mathfrak{g}_{1}$.

The key point is that the above $G_{\mathbf{0}^{-}}$-action and the built-in $\mathfrak{g}$-action on $\operatorname{Ind}_{\mathfrak{g} 0}^{\mathfrak{g}}(V)$ are actually compatible, in the sense that they make $\operatorname{Ind}_{\mathfrak{g}_{0}}^{\mathfrak{g}}(V)$ into a $\left(G_{\mathbf{0}}, \mathfrak{g}\right)$ module, i.e., a module for the super Harish-Chandra pair $\mathcal{P}:=\left(G_{\mathbf{0}}, \mathfrak{g}\right)$. Since $\Psi_{g}\left(\left(G_{\mathbf{0}}, \mathfrak{g}\right)\right)=G$, by Section 4.4 .2 we have that $\operatorname{In} d_{\mathfrak{g}_{0}}^{\mathfrak{g}}(V)$ bears a unique structure of $G$-module which correspond to the previous $\mathcal{P}$-action - i.e., it yields (by restriction and "differentiation") the previously found $G_{0^{-}}$action and $\mathfrak{g}$-action.

Therefore, we define as $\operatorname{Ind}_{G_{0}}^{G}(V)$ the space $\operatorname{Ind}_{\mathfrak{g}_{0}}^{\mathfrak{g}}(V)$ endowed with this $G$-action. One can easily check that this construction is functorial in $V$ and has the universal property which makes it into the adjoint of "restriction" (from $G$-modules to $G_{0^{-}}$ modules), so it has all rights to be called an "induction" functor (from $G_{0}$-modules to $G$-modules).

In addition, if the original $G_{0}-$ module $V$ is faithful, then the induced $G$-module $\operatorname{Ind}_{G_{0}}^{G}(V)$ is faithful too. In particular, this means that if $G_{0}$ is linearizable, then $G$ is linearizable too; more precisely, from a linearization of $G_{\mathbf{0}}$ one can construct (via induction) a linearization of $G$ as well.

4.4.6. Further generalizations. Our construction of a quasi-inverse $\Psi_{g}$ to the functor $\Phi_{g}$ is flexible enough to apply to other contexts. Hereafter we briefly discuss some possibilities.

The non-affine case. We may deal with a more general notion of sHCp, modifying Definition 4.1.2 in one aspect; instead of taking as $G_{+}$any affine group-scheme over $\mathbb{k}$, we drop the "affine" assumption, and allow $G_{+}$to be any group-scheme over $\mathbb{k}$. Correspondingly, we consider also $\mathbb{k}$-supergroup-schemes which are not necessarily "affine", i.e., they are not necessarily representable (as supergroup $\mathbb{k}-$ functors) but still they are obtained by globally pasting together suitable affine $\mathbb{k}$-superschemes (see [6], Ch. 11, §11.1, for a detailed definition). In this more general setup, there still exists a natural functor $\Phi$ from the category of all (not necessarily affine) $\mathbb{k}$-supergroup-schemes to the category of "super Harish-Chandra pairs" in the present, new sense. 
The whole discussion in the present section can then be repeated; in particular, we can construct a functor $\Psi$ from sHCP's to supergroup-schemes, which takes values in the (full sub)category of those supergroup-schemes which are "globally strongly split", in the sense of Definition 3.2.6. The final outcome then will be that the restriction of $\Phi$ to the latter (sub)category and the functor $\Psi$ are quasi-inverse to each other. Therefore, the category of globally strongly split supergroup-schemes (over $\mathbb{k}$, say) is equivalent to the category of sHCp's (over $\mathbb{k}$ ). In a nutshell, Theorem 4.3.14 extends to this more general (non-affine) framework.

Warning: there is just one specific step in the whole procedure, namely Proposition 4.3.13, where (in the proof) we concretely made use of the fact that a given supergroup $G$ under exam was affine, hence the classical subgroup $G_{\mathbf{0}}=G_{+}$in its associated sHCp is affine too. At this point one must definitely adopt a different argument to get the analogous result in the non-affine case.

Dropping finiteness assumptions. Still keeping the assumption that $\operatorname{Lie}(G)=$ $\mathcal{L}_{\mathfrak{g}}$ is representable and $\mathfrak{g}_{1}$ is $\mathbb{k}$-free, one can drop the finiteness assumption on $r k_{\mathbb{k}}\left(\mathfrak{g}_{1}\right)$. In this case, our construction of $G_{\mathcal{P}}$ still makes sense, yielding a supergroup which is automatically fine but is "gs-split" only in a modified sense. Indeed, we have now $G_{\mathcal{P}} \cong G_{+} \times{ }^{i n d} \mathbb{A}_{\mathbb{k}}^{0 \mid d_{-}}$where ${ }^{\text {ind }} \mathbb{A}_{\mathbb{k}}^{0 \mid d_{-}}$is some ind-affine, totally odd superspace, and $d_{-}$is now a possibly infinite cardinal number. As to function algebras, we have $\mathcal{O}\left(G_{\mathcal{P}}\right) \cong \overline{\mathcal{O}\left(G_{+}\right)} \otimes_{\mathbb{k}} \mathcal{O}\left({ }^{\text {ind }} \mathbb{A}_{\mathbb{k}}^{0 \mid d_{-}}\right)$where $\mathcal{O}\left({ }^{\text {ind }} \mathbb{A}_{\mathbb{k}}^{0 \mid d_{-}}\right)$is no longer (a priori) a Grassmann algebra. Our main result - Theorem 4.3.14 - about the equivalence between sHCp's and fine supergroups which are "split" (in a suitable sense) must then be modified accordingly.

On the other hand, Theorem 3.1.10 is proved by Masuoka (see [18], Theorem 4.5) making no special finiteness assumption on commutative Hopf superalgebras. In our language, this means that when $\mathbb{k}$ is a field (with $\operatorname{char}(\mathbb{k}) \neq 2$ ) every affine $\mathbb{k}$-supergroup $G$ is gs-split in the sense of Definition 3.2.6, with no modifications whatsoever! This ought to mean that one should be able to "read" our construction of $G_{\mathcal{P}}$ so as to achieve the same object ${ }^{i n d} \mathbb{A}_{\mathbb{k}}^{0 \mid d_{-}}$, but now presented in such a way that one recognizes it as being a true affine (totally odd) superspace, with $\mathcal{O}\left({ }^{i n d} \mathbb{A}_{\mathbb{k}^{0}}^{0 \mid d_{-}}\right)$now being recognized as a Grassmann algebra. This clarification clearly needs a finer analysis, which goes beyond the goals of the present paper.

Finally, in this "non-finite" setup one can deal with non-affine supergroups; the remarks in the above paragraph (for the non-affine case) apply again, so one ends up with the same conclusions.

The real smooth and complex analytic cases. In the differential setup one studies real Lie supergroups; similarly, in the analytic framework one deals with complex Lie supergroups. In both cases, as it is customary to do, we assume that the superdimension is finite.

In both cases, one can adopt a functorial language, which is strictly close to the one used in the algebro-geometric setup (as we did in the present paper). With such a choice of language - and of technical tools to work with - one can then also reproduce the construction presented in this paper, in particular that of the functor $\Psi_{g}$. The outcome then will be that $\Phi_{g}$ and $\Psi_{g}$ will be equivalences, quasiinverse to each other, between the category of (real or complex) Lie supergroups and the category of (real or complex) sHCp's. Note that here we do not need any "globally strongly split" assumption, since such a property always holds true for real or complex Lie supergroups (see [6], Proposition 7.4.9, and 21] respectively). 


\section{REFERENCES}

[1] Felix Alexandrovich Berezin, Introduction to superanalysis, Mathematical Physics and Applied Mathematics, vol. 9, D. Reidel Publishing Co., Dordrecht, 1987. Edited and with a foreword by A. A. Kirillov; With an appendix by V. I. Ogievetsky; Translated from the Russian by J. Niederle and R. Kotecký; Translation edited by Dimitri Leĭtes. MR.914369 (89b:58006)

[2] Yuri A. Bahturin, Alexander A. Mikhalev, Viktor M. Petrogradsky, and Mikhail V. Zaicev, Infinite-dimensional Lie superalgebras, de Gruyter Expositions in Mathematics, vol. 7, Walter de Gruyter \& Co., Berlin, 1992. MR.1192546 (94b:17001)

[3] Helmut Boseck, Affine Lie supergroups, Math. Nachr. 143 (1989), 303-327, DOI 10.1002/mana.19891430123. MR1018250 (91g:17006)

[4] Helmut Boseck, Lie superalgebras and Lie supergroups. I, Sem. Sophus Lie 1 (1991), no. 2, 109-122. Seminar Sophus Lie (Darmstadt, 1991). MR.1158499(93e:17003)

[5] Helmut Boseck, Lie superalgebras and Lie supergroups. II, Sem. Sophus Lie 2 (1992), no. 1, 3-9. MR 1188627 (94b:17004)

[6] Claudio Carmeli, Lauren Caston, and Rita Fioresi, Mathematical foundations of supersymmetry, EMS Series of Lectures in Mathematics, European Mathematical Society (EMS), Zürich, 2011. MR2840967 (2012h:58010)

[7] Claudio Carmeli and Rita Fioresi, Superdistributions, analytic and algebraic super HarishChandra pairs, Pacific J. Math. 263 (2013), no. 1, 29-51, DOI 10.2140/pjm.2013.263.29. MR3069075

[8] Michel Demazure and Pierre Gabriel, Groupes algébriques. Tome I: Géométrie algébrique, généralités, groupes commutatifs (French), Masson \& Cie, Éditeur, Paris; North-Holland Publishing Co., Amsterdam, 1970. Avec un appendice Corps de classes local par Michiel Hazewinkel. MR0302656 (46 \#1800)

[9] Pierre Deligne and John W. Morgan, Notes on supersymmetry (following Joseph Bernstein), Quantum fields and strings: a course for mathematicians, Vol. 1, 2 (Princeton, NJ, 1996/1997), Amer. Math. Soc., Providence, RI, 1999, pp. 41-97. MR.1701597 (2001g:58007)

[10] R. Fioresi and F. Gavarini, Chevalley supergroups, Mem. Amer. Math. Soc. 215 (2012), no. 1014, vi+64, DOI 10.1090/S0065-9266-2011-00633-7. MR2918543

[11] R. Fioresi and F. Gavarini, On the construction of Chevalley supergroups, Supersymmetry in mathematics and physics, Lecture Notes in Math., vol. 2027, Springer, Heidelberg, 2011, pp. 101-123, DOI 10.1007/978-3-642-21744-9_5. MR2906339

[12] Fabio Gavarini, Chevalley supergroups of type $D(2,1 ; a)$, Proc. Edinb. Math. Soc. (2) 57 (2014), no. 2, 465-491, DOI 10.1017/S0013091513000503. MR3200319

[13] Fabio Gavarini, Algebraic supergroups of Cartan type, Forum Math. 26 (2014), no. 5, 14731564, DOI 10.1515/forum-2011-0144. MR3334037

[14] V. G. Kac, Lie superalgebras, Advances in Math. 26 (1977), no. 1, 8-96. MR0486011 (58 \#5803)

[15] Bertram Kostant, Graded manifolds, graded Lie theory, and prequantization, Differential geometrical methods in mathematical physics (Proc. Sympos., Univ. Bonn, Bonn, 1975), Lecture Notes in Math., Vol. 570, Springer, Berlin, 1977, pp. 177-306. MR0580292 (58 \#28326)

[16] J.-L. Koszul, Graded manifolds and graded Lie algebras, Proceedings of the international meeting on geometry and physics (Florence, 1982), Pitagora, Bologna, 1983, pp. 71-84. MR760837 (85m:58019)

[17] Yuri I. Manin, Gauge field theory and complex geometry, Grundlehren der Mathematischen Wissenschaften [Fundamental Principles of Mathematical Sciences], vol. 289, SpringerVerlag, Berlin, 1988. Translated from the Russian by N. Koblitz and J. R. King. MR.954833 (89d:32001)

[18] Akira Masuoka, The fundamental correspondences in super affine groups and super formal groups, J. Pure Appl. Algebra 202 (2005), no. 1-3, 284-312, DOI 10.1016/j.jpaa.2005.02.010. MR2163412 (2006e:16066)

[19] Akira Masuoka, Harish-Chandra pairs for algebraic affine supergroup schemes over an arbitrary field, Transform. Groups 17 (2012), no. 4, 1085-1121, DOI 10.1007/s00031-012-9203-8. MR3000482 
[20] Akira Masuoka and T. Shibata, Algebraic supergroups and Harish-Chandra pairs over a commutative ring, electronic preprint arXiv:1304.0531 [math.RT] (2013), to appear in Trans. Amer. Math. Soc.

[21] E. G. Vishnyakova, On complex Lie supergroups and split homogeneous supermanifolds, Transform. Groups 16 (2011), no. 1, 265-285, DOI 10.1007/s00031-010-9114-5. MR 2785503 (2012b:58010)

[22] V. S. Varadarajan, Supersymmetry for mathematicians: an introduction, Courant Lecture Notes in Mathematics, vol. 11, New York University, Courant Institute of Mathematical Sciences, New York; American Mathematical Society, Providence, RI, 2004. MR2069561 (2005g:58011)

Dipartimento di Matematica, Università di Roma "Tor Vergata", Via della ricerca SCIENTIFICA 1, I-00133 Roma, ITALY

E-mail address: gavarini@mat.uniroma2.it 